Article

\title{
Kinetics of Thermally Activated Physical Processes in Disordered Media
}

\section{Bertrand Poumellec and Matthieu Lancry*}

Institut de Chimie Moléculaire et des Matériaux d'Orsay, Université Paris Sud, UMR CNRS-UPSud 8182, Orsay Center, 91405 Orsay, France; E-Mail: bertrand.poumellec@u-psud.fr

* Author to whom correspondence should be addressed; E-Mail: matthieu.lancry@u-psud.fr.

Academic Editor: Stephen C. Bondy

Received: 07 January 2015 / Accepted: 01 April 2015 / Published: 15 July 2015

\begin{abstract}
We describe a framework for modeling the writing and erasure of thermally-distributed activated processes that we can specifically apply to UV-induced refractive index change, particularly in fibers. From experimental measurements (isochrons and/or isotherms), this framework allows to find the distribution function of the activation energy by providing only a constant, which can be determined by a simple variable change when a few assumptions are fulfilled. From this modeling, it is possible to know the complete evolution in time of the system. It is also possible to determine the annealing conditions for extending a lifetime. This approach can also be used for other physical quantities, such as photodarkening, stress relaxation, and luminescence decay, provided that it can be described by a distribution function.
\end{abstract}

Keywords: glasses; photodarkening; radiation-induced attenuation; fiber Bragg gratings; and lifetime prediction

\section{Problem: Dynamic Evolution of Physical Quantities in Disordered Media}

UV-written intracore Fiber Bragg gratings are now routinely used for applications in optical telecommunications or as sensors in civil engineering. Most applications require a long grating lifetime. For example, in dense wavelength-division multiplexing (DWDM) optical communication systems, the grating-based devices should continue working at an agreed specification for 25 years in the temperature 
range $-40{ }^{\circ} \mathrm{C}<\theta<80{ }^{\circ} \mathrm{C}$. Accordingly, several theoretical and/or experimental studies, dealing with accelerated lifetime tests, have been carried out with a view to forecast possible degradation of the UV-induced refractive index changes [1,2].

For modeling the relaxation processes in disordered media, several approaches can be used and an extended report can be found in the reference book of Richert and Blumen [3]. The main problem is to account for the effect of disorder on thermally activated processes. The disorder can have an impact on the various steps of the physico-chemical reaction, leading to a change in the observed quantity (e.g., index changes, radiation-induced losses, etc.). For instance, if hopping is concerned in the reaction, the disorder will appear in the hopping distance or in the waiting time, if an energy barrier is involved, the activation energy will be distributed because the transition and/or the stable state configurations are varied.

These two examples differ fundamentally on one point: the temperature will not affect, in the same way, the range limit or the temperature-activated hopping. In particular, the dependence on the stability of refractive index with the writing or the ageing temperature [4] can be only explained by processes with distributed activation energies. Other arguments connected to changes in the structure reinforce this conclusion. However, a process like decoration of defect networks with $\mathrm{H}$ will not necessarily be relevant to a thermally activated process. In this paper, we consider only processes involving the distribution of activation energies as it is seen that this approach yield good results $[1,5,6]$ with regards to the problem of index stability.

\subsection{Under an External Action}

Maybe the most important example of distributed processes for people working in the fabrication of telecommunication devices is the non-exponential growth of refractive index change under UV irradiation. In Figure 1 we can see that for a pulsed $248 \mathrm{~nm}$ laser, the index change grows according to a power law with an exponent of 0.5 [7]. In a number of fibers, this law is followed with an exponent between 0.25 and 0.8. We know from [8] that an index change in Ge-doped glasses is achieved by UV induced compaction. It is known that it is possible to achieve compaction in pure silica by the same means [9], but it is also possible to obtain compaction by ion or electron irradiation. Primak [10] is probably the author who studied these processes the most.

We can see in Figure 2 that compaction follows a power law again, with an exponent of 0.66. More recently, Allan et al. [11] showed that $193 \mathrm{~nm}$ irradiation in pure silica leads to densification by two-photon absorption (Figure 3).

We note in this case that the dose is no longer the relevant parameter but $I^{2} N$, where $I$ is the power density and $N$ is the pulse number. The behavior is a power law with an exponent of 0.7 .

Another example is the IR darkening of the Ge/Al/Er-codoped core optical fibers, observed under an $\mathrm{H}_{2}$ atmosphere of $0.012 \mathrm{~atm}$, as shown in Figures 4 and 5. Lemaire et al. [12] showed that $\mathrm{OH}$ vibration absorption at $1.38 \mu \mathrm{m}$ also follows a power law in the same kind of experiment, the exponent of which increases linearly with temperature. Moreover, they have observed that the logarithm of the pre-exponential factor also evolves linearly with temperature. The power law is not the only behavior recorded in $\mathrm{H}_{2}$-induced IR darkening of fibers. Similar sensitivity of erbium-doped fibers to hydrogen has been reported in [13]. Rush et al. [14] showed that, in the case of phosphorus doped core optical fibers, a $\log t$ law leads to a better fit in a larger range of time (Figure 6). The same type of law has been 
invoked in transient creep experiments in metals. In this case, a constant distribution of sites is inferred [15].

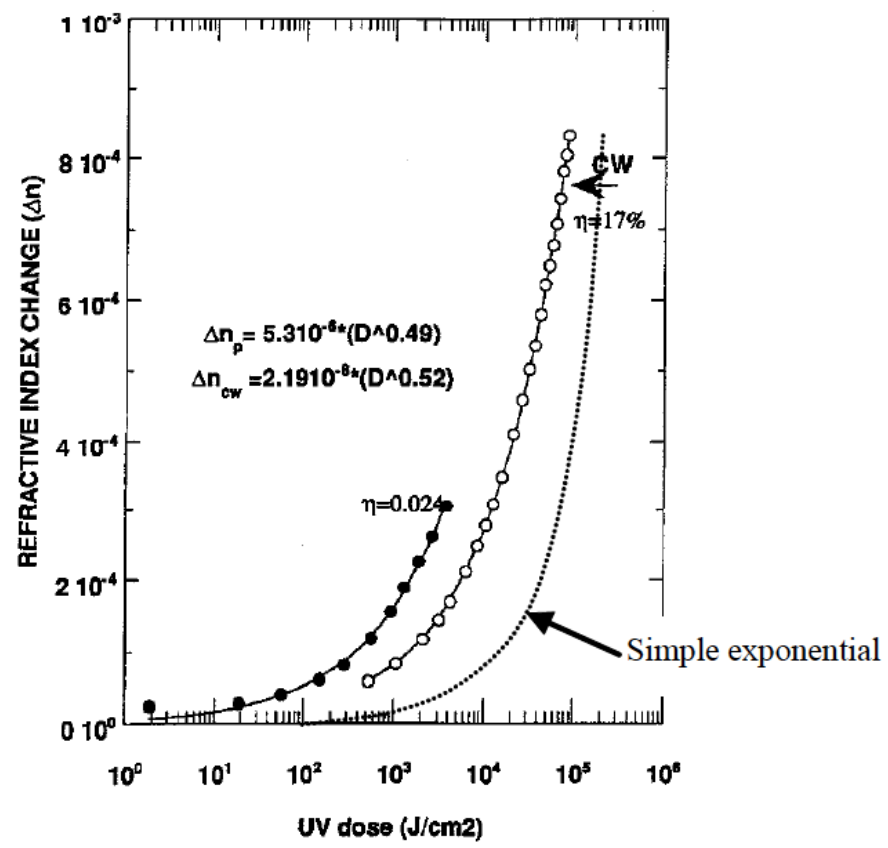

Figure 1. Index change under UV irradiation, reprinted with permission from [7]. Copyright 1996 Elsevier.

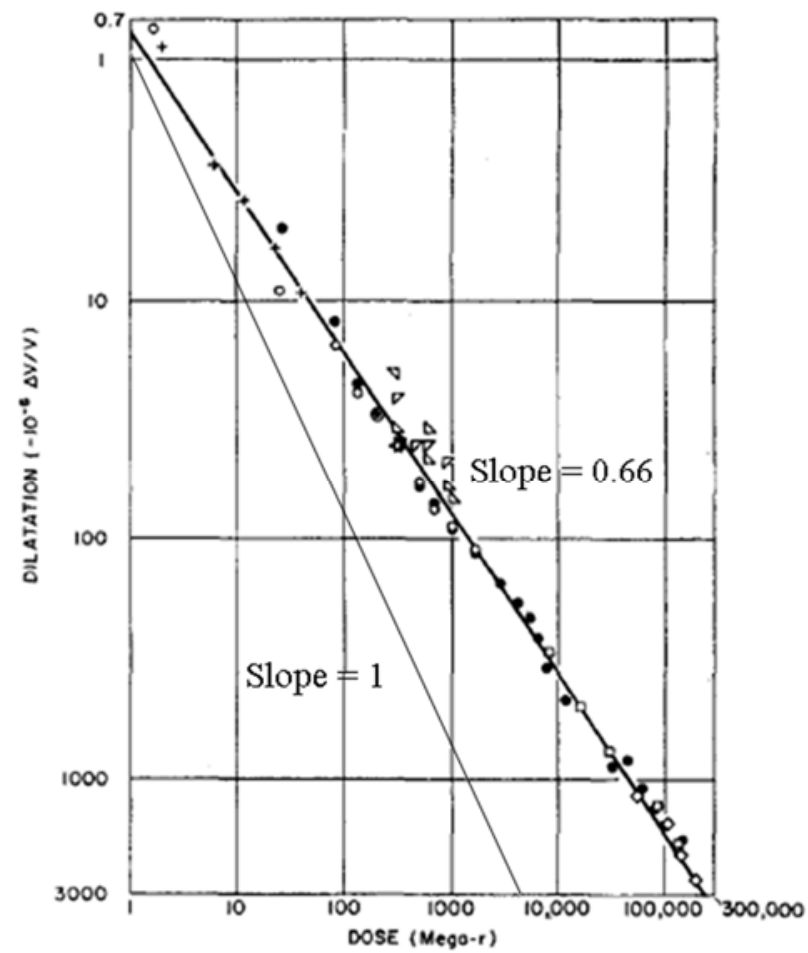

Figure 2. Electron irradiation induced compaction, reprinted with permission from [10]. Copyright 1968 AIP. 


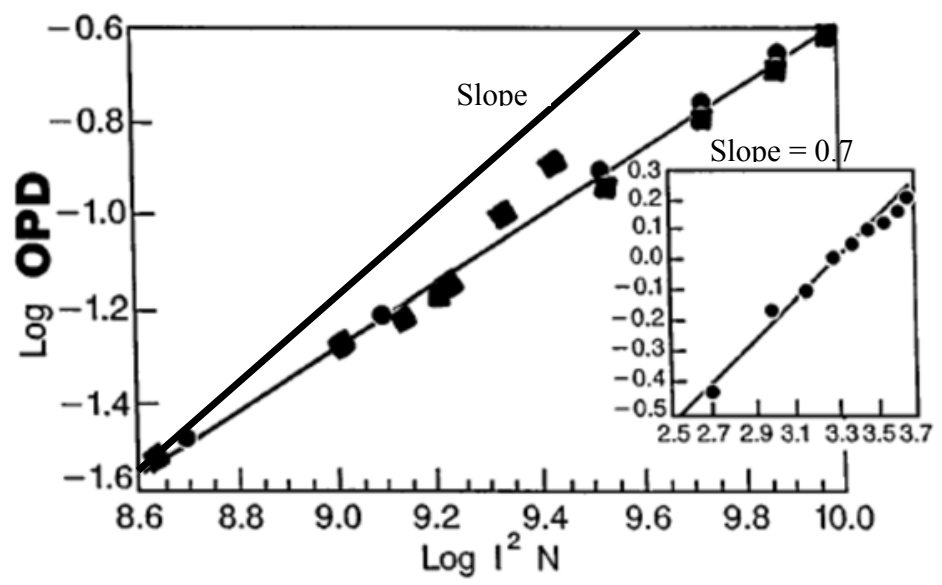

Figure 3. Densification in fused silica under $193 \mathrm{~nm}$ irradiation, reprinted with permission from [11]. Copyright 1996 OSA. The optical path difference follows: $O P D=a\left(I^{2} N\right)^{0.7}$.

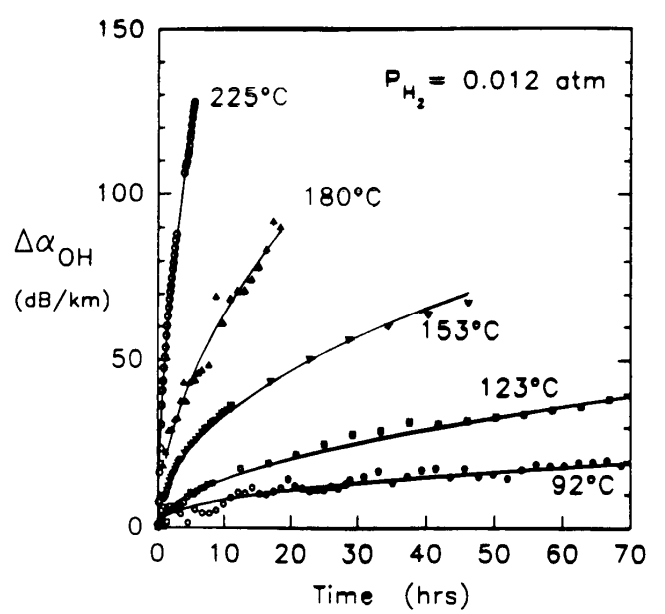

Figure 4. IR darkening of the Ge-doped core optical fiber, reprinted with permission from [12]. Copyright 1991 SPIE. The $\mathrm{OH}$ growth data are well described by an equation of the form: $\Delta \alpha_{\mathrm{OH}}=a(t)^{m}, \quad a=a_{0} \exp \left(T / T_{0}\right)$.

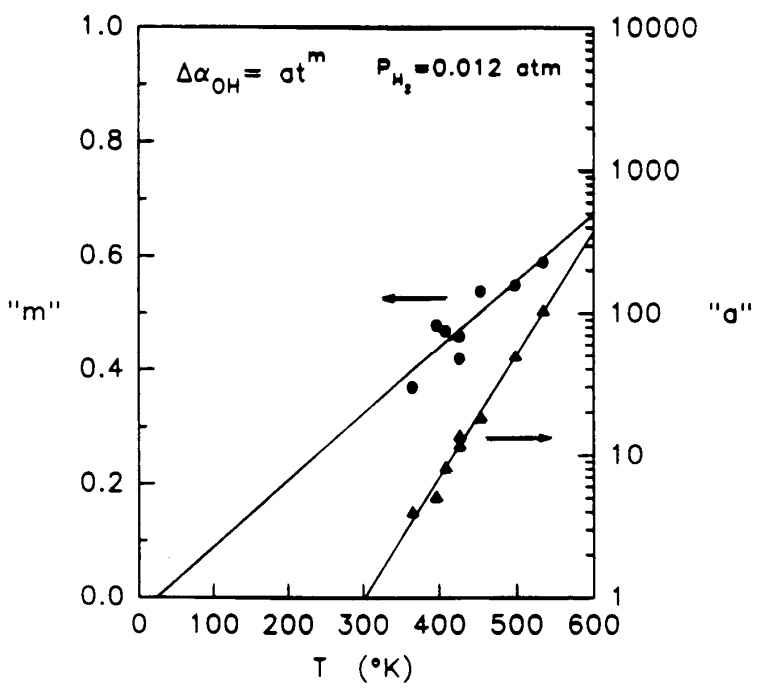

Figure 5. $a$ and $m$ coefficients deduced from non-linear data fitting procedure. 
Last example for completing the panel of non-exponential behavior of systems submitted to an external action is the bleaching of the $400 \mathrm{~nm}$ luminescence by $266 \mathrm{~nm}$ light in Ge-doped core optical fibers (Figure 7). In that case, a stretched exponential law is used $\left(\exp \left(-\alpha t^{\beta}\right)\right)$ and the exponent $\beta$ is found depending linearly on power density [16]. Of course, this list is not exhaustive and is just made to show the spreading of the non-exponential behavior. It is valuable to see that non-exponential processes occur also spontaneously, without any external action.
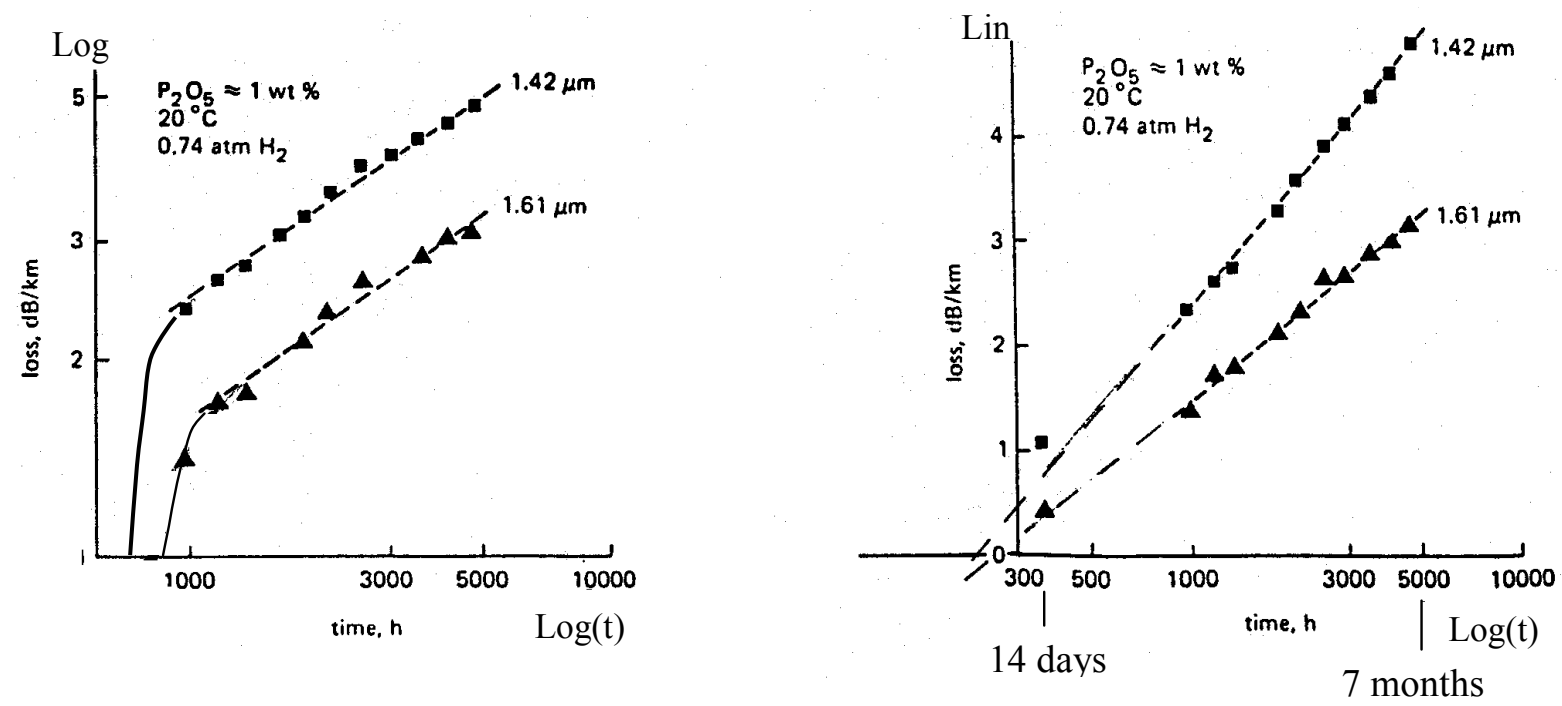

Figure 6. IR Hydrogen induced darkening, reprinted with permission from [14]. Copyright 1983 IEEE.
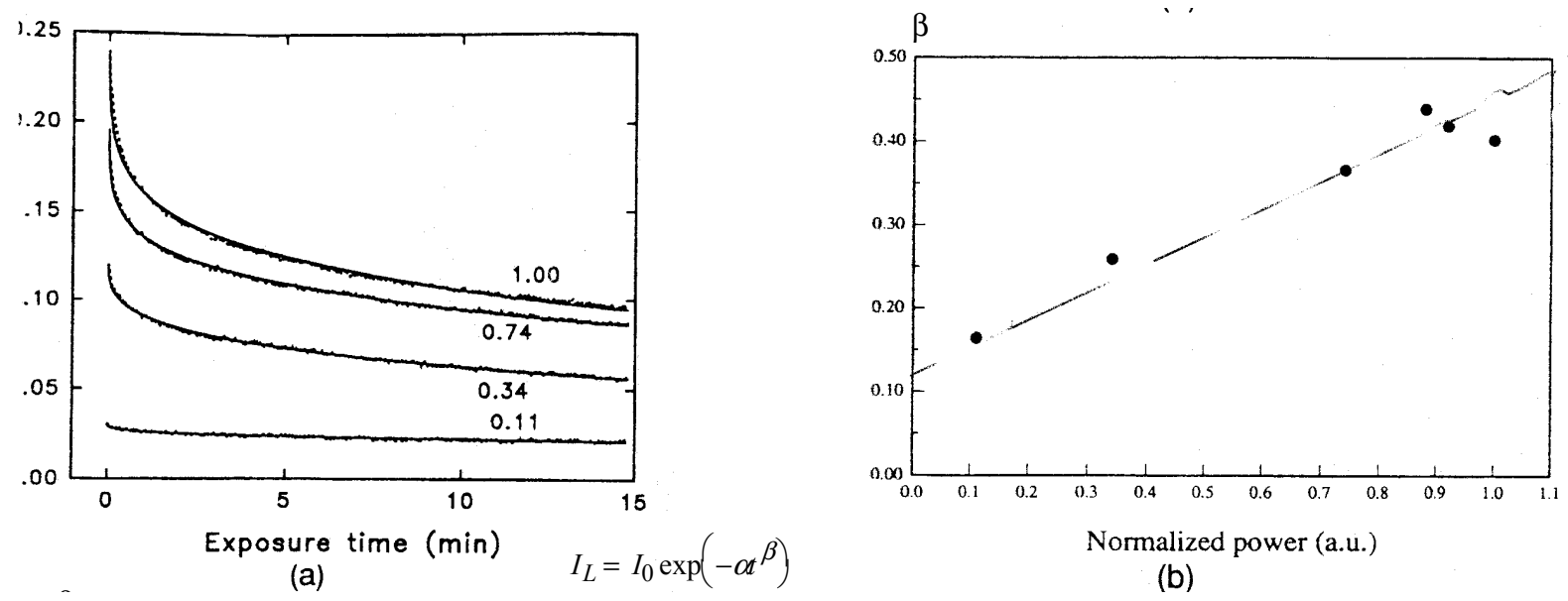

(b)

Figure 7. (a) Bleaching of $400 \mathrm{~nm}$ luminescence by $266 \mathrm{~nm}$ light in Ge-doped $\mathrm{SiO}_{2}$ fibers, reprinted with permission from [16]. Copyright 1993 OSA; (b) Exponent $\beta$ of the stretched exponential law according to the power density.

\subsection{Thermal Relaxation}

Of course, the most well known example in our field is the UV-induced refractive index change relaxation (Figure 8). The most popular law in this field is the inverse power law or sigmoidal law, studied by Erdogan et al. [1,17]: 1/(1+at $\left.{ }^{\alpha}\right)$. It is based on an energy distribution of trapping sites, 
according to a bell-shaped curve with a suitable analytical form. In that case, the authors showed that exponent $\alpha$ is linearly dependent on temperature (as in the case of $\mathrm{H}_{2}$-induced IR darkening).

Close to UV-induced refractive change, the encoded SHG by irradiation, with a fundamental $1.064 \mu \mathrm{m}$ and second harmonic of $532 \mathrm{~nm}$, is found to decrease following a stretched exponential law [18].

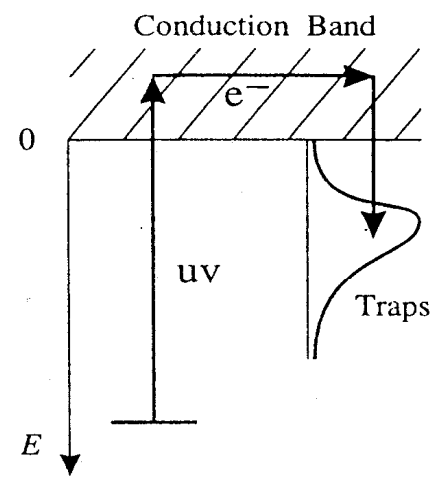

(a)

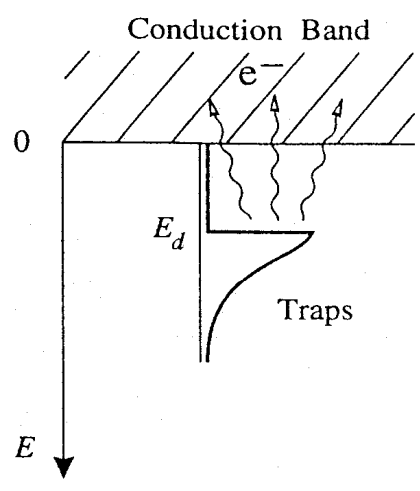

(b)

Figure 8. Erdogan model, reprinted with permission from [1,17]. Copyright 1994 OSA. (a) Uniform population regardless of the trapping energy depth; (b) Progressive trap depopulation according to the trapping energy.

Irradiation with more an energetic photon (gamma) induces optical losses following a power law in classical SMF28 Corning fiber [19]. This photodarkening relaxes following an $n$ order kinetics according to the authors, which has no physical basis but plays the role of non-exponential kinetics (Figure 9).
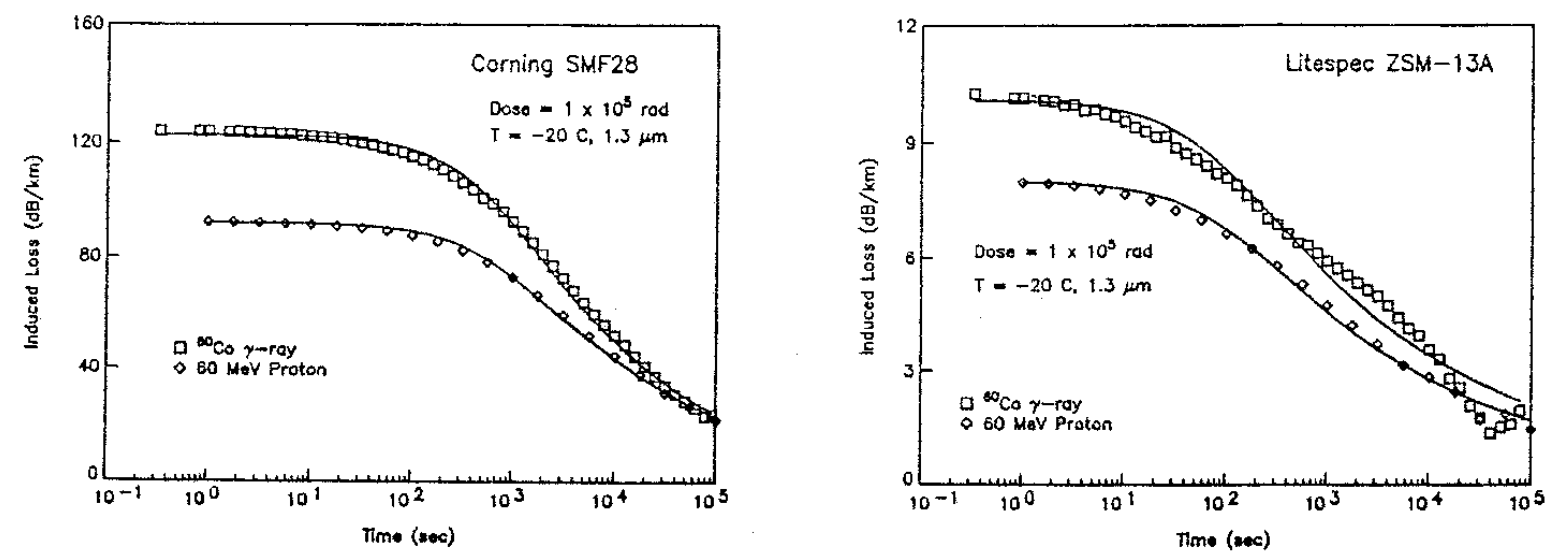

Recovery of the loss induced in (a) Corning SMF-28 and (b) Litespec ZSM single mode fibers by an ionizing dose of $10^{5} \mathrm{rad}(\mathrm{Si})$ from either a ${ }^{60} \mathrm{Co} \gamma$-ray source or protons from a cyclotron.

Figure 9. Recovery of gamma induced losses in fibers, reprinted with permission from [19]. Copyright 1996 OSA.

A part of the optical losses induced by gamma irradiation is the production of non-bonding oxygen hole centers, i.e., a pending bond on non-bridging oxygen. Deparis et al. [20] showed that a good fit is obtained of the recovery, using a peculiar stretched exponential function (Figure 10): Loss $=\operatorname{Loss}_{0} \exp \left(-\frac{t}{\tau_{0}}-\left(\frac{t}{\tau}\right)^{3 / 2}\right)$. 
The same kind of recovery is the luminescence collected after, for instance, $\mathrm{Cr}^{3+}$ excitation in alkaline disilicate glasses. The number of photons collected is the result of the relaxation of excited sites having various lifetimes due to various environments of the $\mathrm{Cr}$ ion. Therefore, short lifetimes are seen at the beginning and long ones at the end (Figure 11 [21]).

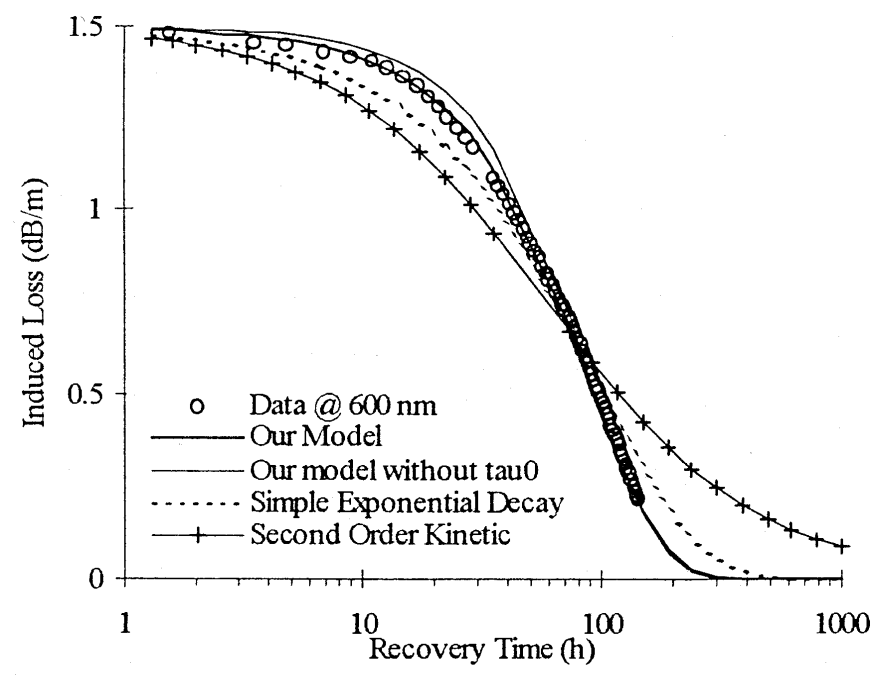

Figure 10. $\mathrm{NbOHC}$ band recovery after gamma irradiation, reprinted with permission from [20]. Copyright 1996 Elsevier.

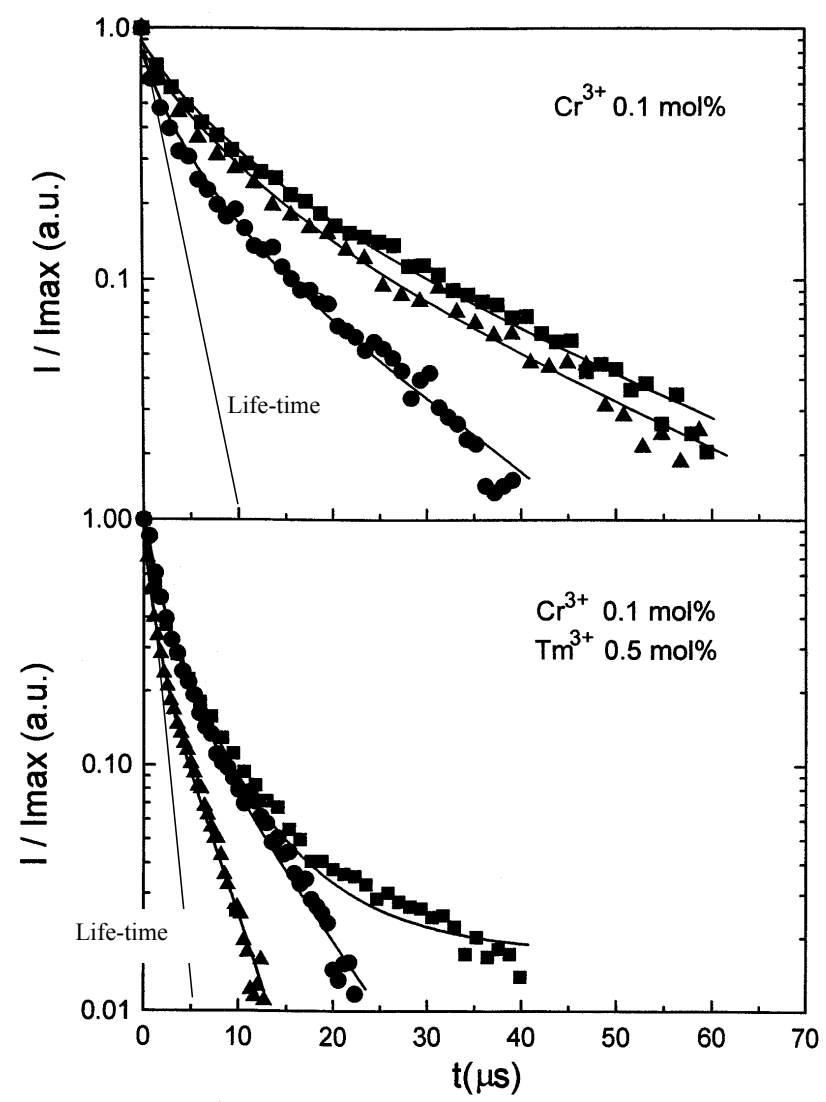

Figure 11. ${ }^{4} \mathrm{~T}_{2} \rightarrow{ }^{4} \mathrm{~A}_{2}$ emission from $\mathrm{Cr}^{3+}(735 \mathrm{~nm})$, reprinted with permission from [21]. Copyright 1997 Elsevier. 
This excitation is of the same nature as electron excitation followed by trapping (Figure 12). Don Monroe [22] showed that the non-exponential behavior of the electric current, due to relaxation of trapped electron after $1.6 \mathrm{eV}$ excitation in $\mathrm{As}_{2} \mathrm{Se}_{3}$, is due to a distribution of trapping sites according to a Poisson distribution and corresponding to a band tail trapping.

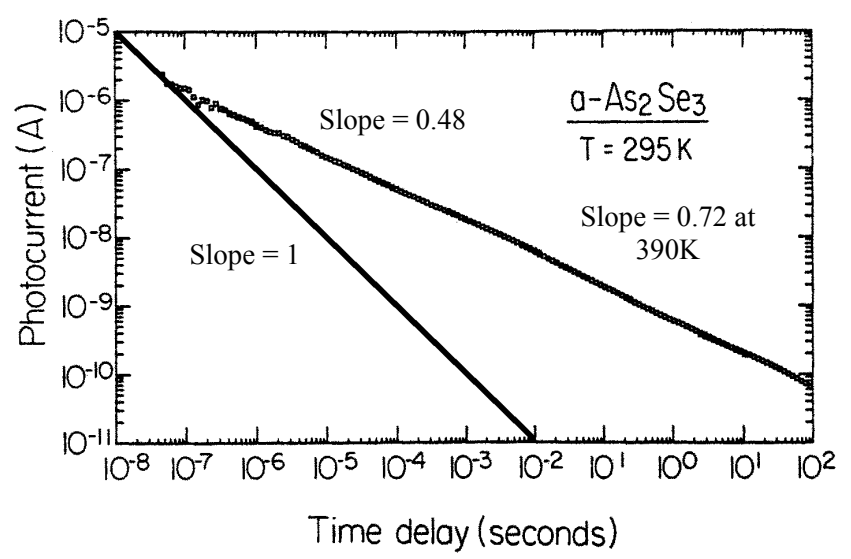

Figure 12. Band tail photocurrent in $\mathrm{As}_{2} \mathrm{Se}_{3}$ glass. $i=a t^{\alpha}$, the exponent varies on $T$ as $\left(T-T_{0}\right) / T_{0}$. A Poisson distribution of trapping sites is deduced.

Non-exponential relaxation occurs not only after irradiation. Figure 13 from Kroide et al. [23] is just an example that viscosity below glass transition temperature (i.e., relaxation of glass after a stress perturbation) is also of the same nature. Stretched exponential behavior is found with an exponent smaller than one, as usual. The exponent seems to depend on the fictive temperature of the glass. This is relevant of a change of atomic configurations in glasses, from site to site, toward a more stable arrangement. Similar processes occur in polybutadiene due to cis-trans isomerization [24].

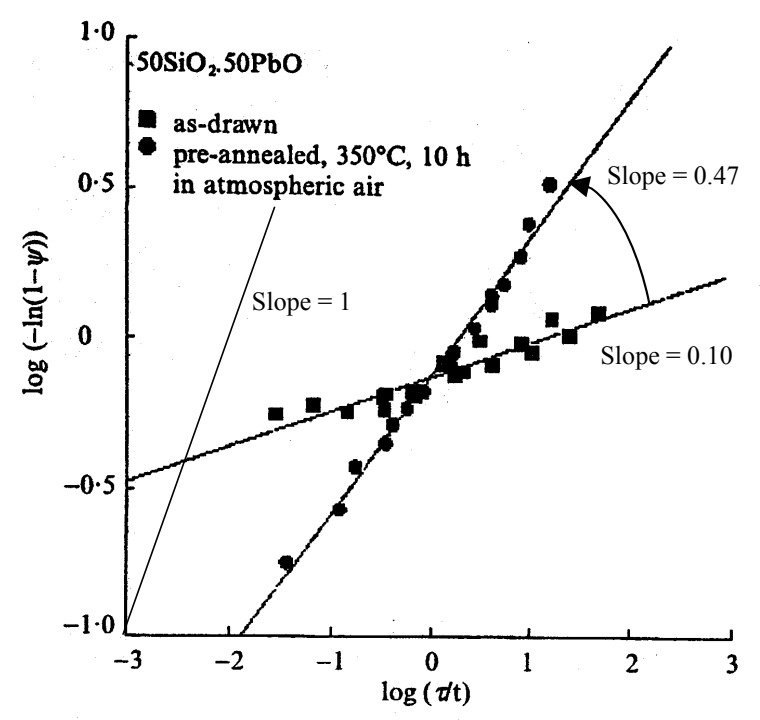

Plot of $\log (\mathrm{t} / \tau)$ versus $\log (-\ln (1-\psi))$ for SP55 glass

Figure 13. Viscosity below glass transition temperature, reprinted with permission from [23].

Copyright 1997 Society of Glass Technology. $\eta_{\text {apparent }}=\frac{1-\psi}{3 d \psi / d t}$ with $1-\psi=\exp \left(-(t / \tau)^{b}\right)$. 
Finally, the last example shows that viscosity relaxation leads to stress relaxation especially in optical fiber after drawing. Mohanna et al. [25] found that stress decrease obeys to a stretched exponential with an exponent increasing linearly on temperature (Figure 14) as it was for $\mathrm{H}_{2}$ induced IR darkening (Figure 4).
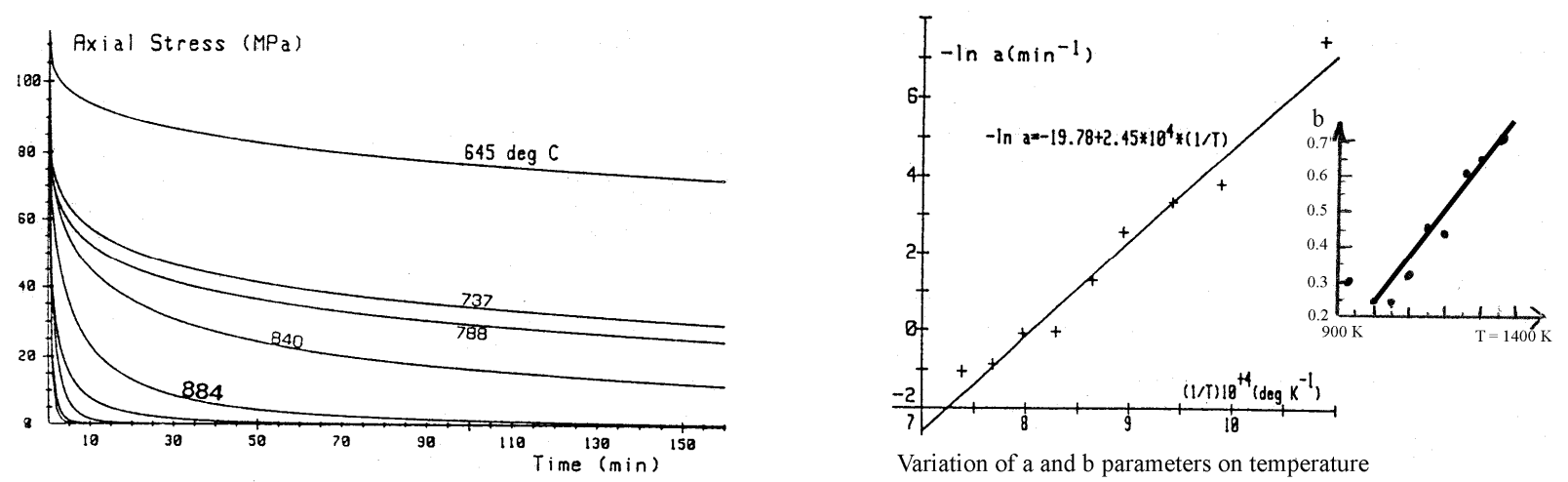

Variation of $\mathrm{a}$ and $\mathrm{b}$ parameters on temperature

Figure 14. Stress relaxation in Ge-doped fibers, reprinted with permission from [25]. Copyright 1990 IEEE. The equation is $\sigma=\sigma_{0} \exp \left(-(a t)^{b}\right)$.

In the following section, we describe the approach in the frame of a distributed activation energy case, which can be used to model the various experimental behaviors shown above. This theory is applied if the distribution function does not change along the reaction, i.e., if the medium or the long-range order of the glass structure is not modified during the reaction (in other words, if the reaction is local). If not, extension of the theory can be developed, as shown later.

\section{Modeling}

The method, now widely used, and introduced in our field by Lemaire et al. in 1984 [26], for hydrogen induced darkening, and Erdogan et al. in 1994 [1,17], for Fiber Bragg grating stability, is based on numerous previous works.

The history begins with Kohlrausch in 1847 [27], studying the electric discharge of a Leyde bottle. He found a stretched exponential behavior with an exponent of 0.43. Later in 1876, Hopkinson proposed another relaxation function for dielectric: $\mathrm{Bt}^{-n}$. In 1893, Wiechert suggested that relaxation energy in solids is distributed according to a Gaussian law. In 1907, Von Schweidler introduced the concept of relaxation time. In 1913, Wagner suggested that relaxation time was governed by a probability function that was actually Gaussian. At that date, everything was available for allowing Vand [28] to propose two experimental methods for studying the activation energy barrier distribution: the isothermal and tempering annealing. He defined, from first order kinetics, a cutting energy, showed that the distribution function is the derivative of the physical quantity according to this energy, and studied the error introduced by this approximation. This really was an important step for the study of disordered media. Then, in 1955, Primak [29] applied the Vand approach to kinetic orders larger than one, but he noted that one-order kinetics are most likely in solids. In 1960, Primak [30] goes on in studying the isochronous method. He performed a great deal of experiments in silica, especially regarding radiation-induced compaction or dilatation. He slightly improved the Vand method. After then, several publications dealing with distributed kinetics appeared, but without mentioning the origin. Tiedje and Orenstein (1980) [31] invoked a demarcation energy, which is the Vand cutting energy. In 1986, Monroe et al. [22] use 
previous work for the interpretation of the photocurrent decay in selenium arsenide. In 1991, Miller [32] developed a predictive formalism to describe generalized activated physical processes. Lemaire, in 1992 [33], and Erdogan, in 1994 [1,17], applied the method to optical fibers. In 1996, Van den Brink [34] defined a master curve in viscoelastic relaxation using several Maxwell elements (simple exponentials). In 1997, Kannan et al. [35] used this master curve concept for predictive ends in optical fibers.

We are now describing the theory of distributed kinetics, allowing to analyze experimental results, however, before that, let us understand the difference between a non-distributed kinetic (simple reaction pathways - SIREPA) and a distributed one (variable reaction pathways_-VAREPA).

\subsection{Exponential versus Non Exponential Kinetics (Introduction to the Theory)}

Exponential kinetics are the time behavior of a first order reaction. We can write: $\mathrm{A} \stackrel{k}{\longrightarrow} \mathrm{B}, E_{0}, k=k_{0} \exp \left(-\frac{E_{0}}{K_{\mathrm{B}} T}\right)$, where $E_{0}$ is the unique activation energy of the reaction, i.e., the energy barrier to transform $\mathrm{A}$ in $\mathrm{B}$ in Figure 15. The rate equation for this reaction is $\frac{d \mathrm{~A}}{d t}=-k \mathrm{~A}, \mathrm{~A}+\mathrm{B}=\mathrm{A}_{0}$. The computation of the $\mathrm{A}$ or $\mathrm{B}$ contents leads to the following expressions: $\mathrm{A}=\mathrm{A}_{0} \exp (-k t) ; \mathrm{B}=\mathrm{A}_{0}[1-\exp (-k t)]$. We can notice that, if we plot the quantity B versus time (Figure 16a), the curve will exhibit a linear behavior at the origin before saturation. If we plot $\log \left(\mathrm{A}_{0} /\left(\mathrm{A}_{0}-\mathrm{B}\right)\right)$ against time (Figure $\left.16 \mathrm{~b}\right)$, we will get a linear behavior until infinity.

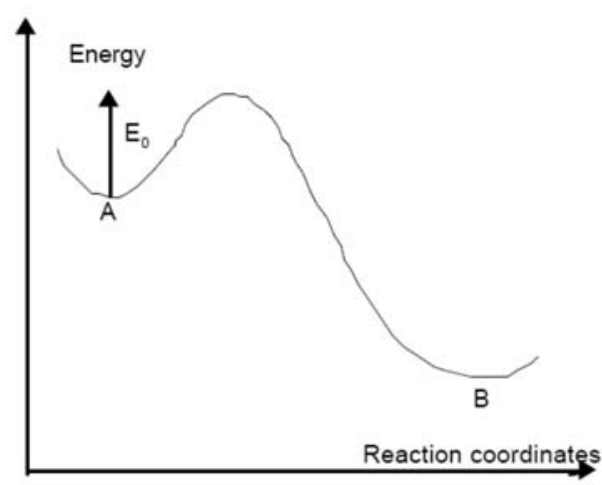

Figure 15. Chemical pathway between $\mathrm{A}$ and $\mathrm{B}$.

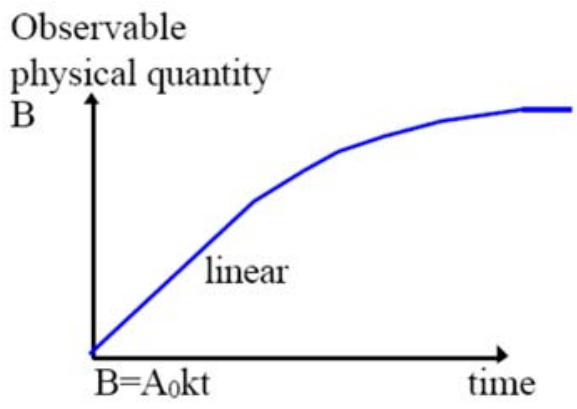

(a)

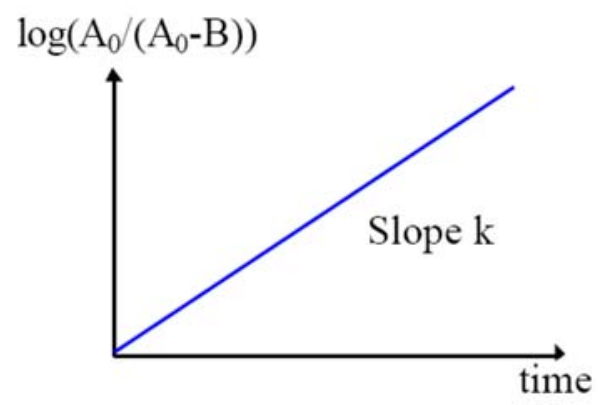

(b)

Figure 16. SIREPA. (a): $\mathrm{B}$ vs. time; $(\mathbf{b}): \log \left(\mathrm{A}_{0} /\left(\mathrm{A}_{0}-\mathrm{B}\right)\right)$ vs. time. 
A non-exponential behavior, such as a $t^{\alpha} /\left(1+\mathrm{a} t^{\alpha}\right)$, is quite different. If we plot B versus time (Figure 17a), the curve exhibits an infinite slope at the origin, then the slope decreases strongly, and finally the evolution is slower than for an exponential behavior. Translated in term of an exponential equation, we can see that the rate constant $k$ decreases on time, this means that there is a strengthening of the kinetics. If we plot $\log \left(\mathrm{A}_{0} /\left(\mathrm{A}_{0}-\mathrm{B}\right)\right)$ versus time, Figure $17 \mathrm{~b}$, the plot is not at all linear, but exhibits an infinite slope at the origin and then the slope decreases strongly.

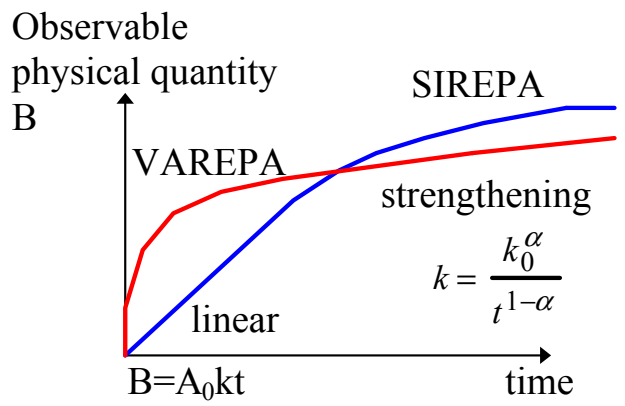

(a)

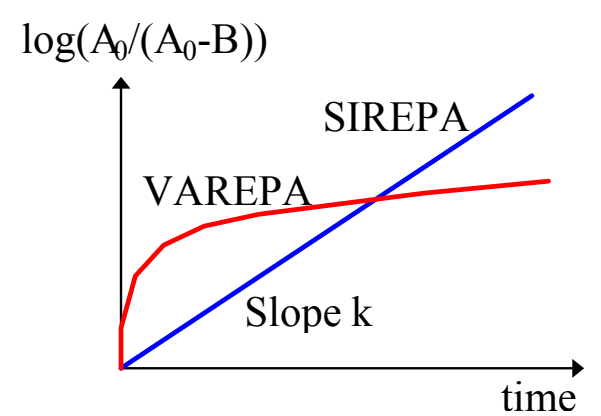

(b)

Figure 17. (a) $\mathrm{B} v s$. time; (b) $\log \left(\mathrm{A}_{0} /\left(\mathrm{A}_{0}-\mathrm{B}\right)\right)$ vs. time.

Another plot usually performed in this case is a log-log plot (Figure 18). In this frame, the exponential equation will exhibit a slope of unity at the origin whereas the power law will have a slope $\alpha$ usually smaller than 1.

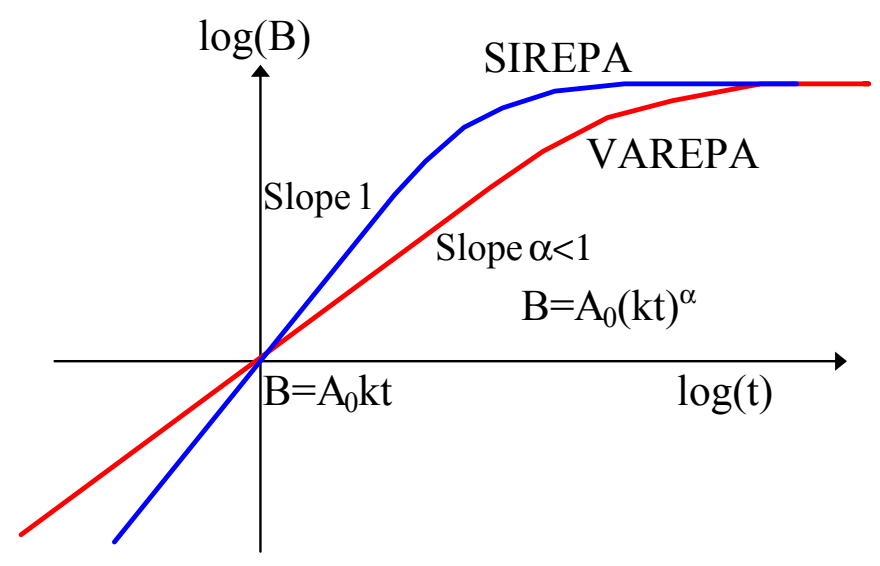

Figure 18. VAREPA kinetics compared SIREPA. $\log (\mathrm{B})$ vs. $\log ($ time $)$.

The thermal behavior of these two kinds of kinetics is subtler. The reverse reaction (if any) will work through energy barriers complementary to the formation energy difference between A and B species (see Section 4.1). The two kinetics can sometimes be differentiated by their thermal relaxation. In an isochronous plot ( $\mathrm{B} / \mathrm{B}_{0}$ versus $T$ for a given $\left.\Delta t\right)$, Figure 19 , the exponential behavior will give rise to the same sigmoidal plot whatever the temperature or the duration of the writing (reaction $\mathrm{A} \rightarrow \mathrm{B}$ ). For a non-exponential behavior, the stability increases with writing temperature (until a limit) and stability increases as well with the writing time. 


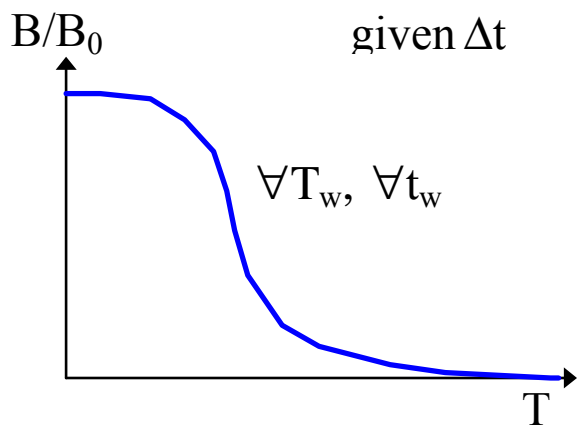

(a)

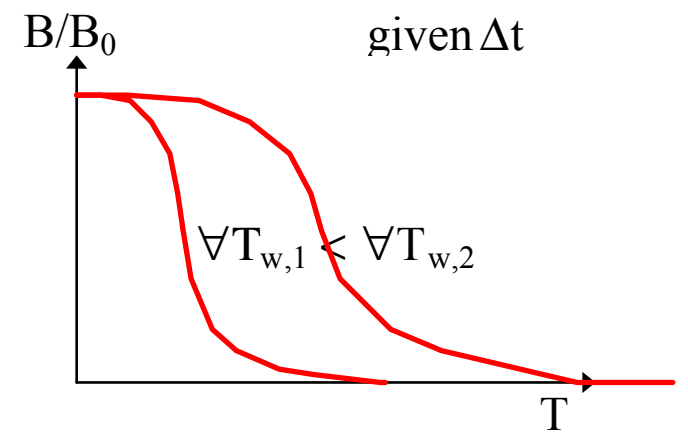

(b)

Figure 19. Isochrons curves. (a) SIREPA; (b) VAREPA.

\subsection{Kinetics of Writing}

We will now move on to model the kinetics of writing. This is based on several assumptions.

\subsubsection{First Group of Assumptions}

(1) We can easily imagine that the reaction leading to refractive index change contains an absorption step on a species (for instance Oxygen Deficient Center), followed sometimes by a relaxation to another excited level (Figure 20). Then, several pathways are possible (one to luminescence, another one to bond breaking, migration, and structural rearrangement). All of them finally contribute to refractive index change. The first assumption we make here is that only one elementary reaction is involved: the limiting reaction of the process. We can write the reaction $\mathrm{A} \rightarrow \mathrm{B}$.

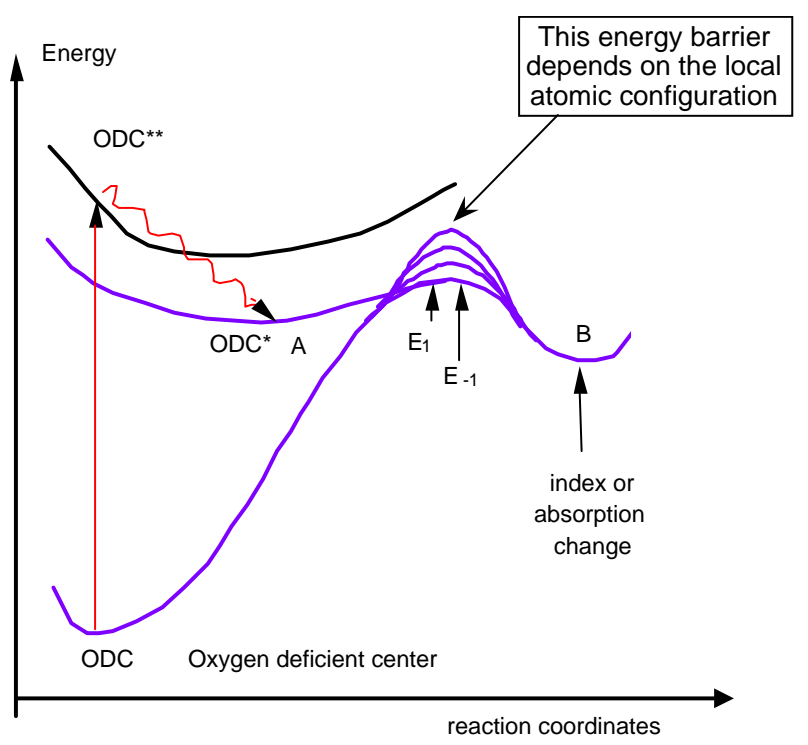

Figure 20. Example of the absorption step of a physical chemical reaction, followed by a thermally activated reaction.

(2) The second assumption is that it is thermally activated (with an activation energy E). The Arrhenius law can be used, the $\mathrm{d}[B] / \mathrm{d} t$ rate is proportional to a reaction constant $k_{0}$ like 
$k(E, T)=k_{0} \exp \left(-E / k_{\mathrm{B}} T\right)$, where $k_{\mathrm{B}}$ is the Boltzman constant and $[B]$ is the concentration of the B species.

(3) The third assumption is that activation energy is distributed. As a matter of fact, in glasses, $E$ is not unique. The creation energy of B can vary with its atomic environment. The transition state energy (the bottleneck on the reaction pathway) is also sensitive to the disordered environment in such a way that the activation energy, which is the energy difference between $B$ and the transition state, is distributed. This means that chemical pathways are variable depending on the configuration distribution of the transition state and/or the initial stable states throughout the glass. This is at the origin of ergodicity lost because space-time points are no more equivalent. The reaction is faster in some places than in others. This is also heterogeneity, but not at a macroscopic level, just at a microscopic one.

We can call the relevant distribution $g(E)$ and we chose to normalize it. If $g$ is relevant to a thermal disorder, a Gaussian distribution seems likely. If $g$ is relevant to a diffusional process, a Poisson distribution is better, but other approximations have also been used: differentiation of a sigmoid [1,17], and top hat function [5]. We will also see that $g(E)$ can be obtained from the experiment and then approximated if necessary. At this step of the description, calling $x$ the advancement of the reaction, depending on the activation energy $E$ and the time, we have:

$$
\left.\frac{\partial x(E, t, T)}{\partial t}\right)_{E, T}=k(E, T)(1-x(E, t, T))[B](t, T)=A_{0} \int_{0}^{\infty} x(E, t, T) g(E) d E
$$

These assumptions constitute the first group of assumptions that are mainly connected to disorder.

\subsubsection{The Assumption on the Reaction (Connection with Time and Temperature)}

This assumption will allow computing the time dependence properly.

Most of the elementary reactions are first order, some are second order, when two species associate, but these are less likely. Thus, it is reasonable to assume that $\mathrm{A} \rightarrow \mathrm{B}$ is a first order reaction with a rate constant $k(E, T)=k_{0} \exp \left(-E / k_{\mathrm{B}} T\right)$ (second order reaction is investigated in Section 5.1). This allows to compute the advancement degree $x(E, t, T)=1-\exp (-k(E, T) t)$. Usually, the $\mathrm{B}$ integral is not analytically computable and, thus, extraction of $g(E)$ is very difficult. A simplification of the integral is required to handle complex cases. Here, we can observe that $x(E, t, T)$ is a very steep function of $E$, in such a way that, below a demarcation energy, $E_{d}, x$ is equal to 1 and above $E_{d}$ is equal to 0 (Figure 21). This clever remark, made by Vand [28], leads to a useful simplification of the equation:

$$
[B](t, T)=A_{0} \int_{0}^{E_{d}(t, T)} g(E) d E=[B]\left(E_{d}\right)
$$

We note that B content appears to depend now only on $E_{d}$.

To define $E_{d}$ properly, we can chose the value where $x(E)_{t, T}$ varies the most (the steepest variation defined by $\left.\frac{\partial^{2} x}{\partial E^{2}}\right)_{t, T}\left(E=E_{d}\right)=0$, in this case, we get $E_{d}=k_{\mathrm{B}} T \ln \left(k_{0} t\right)$. 


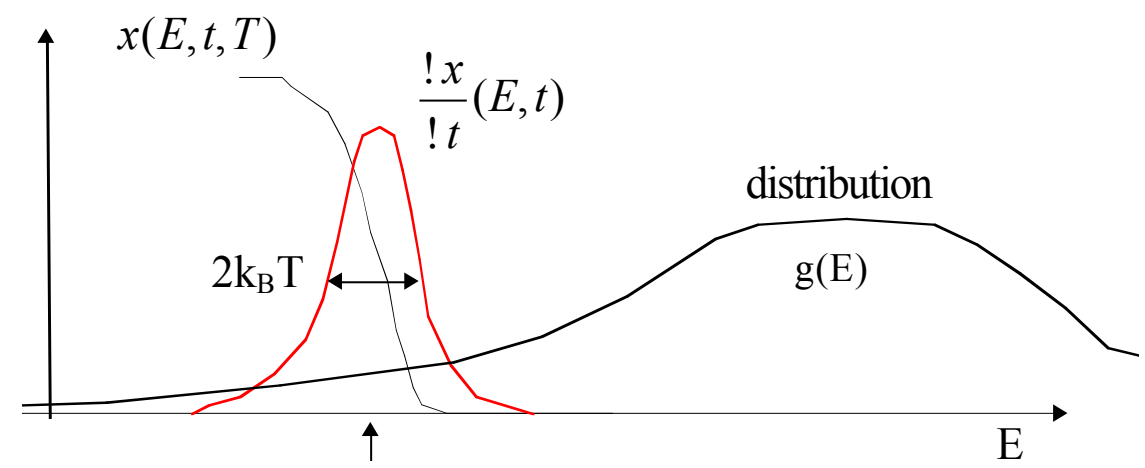

For a given $\mathrm{t}$

Figure 21. Comparison of widths between $x(E, t, T)$ and the distribution $g(E)$.

Remarks and consequences of the theory:

(1) It is worth noting that the reaction properties appear now only in $E_{d}(t, T)$, on one hand (the bound of the integral), and, on the other hand, the structural disorder appears in the integrand.

(2) Note that the demarcation energy approximation is valid only if $g(E)$ varies slower than $x(E, t, T)$. The width of $\left.\frac{\partial x(E, t, T)}{\partial E}\right)_{t, T}$ is $2 k_{B} T$. Otherwise, a correction has to be added to the integral [32]:

$$
-\left.k_{\mathrm{B}} T \sum_{k=0}^{k=\infty} c_{k+1} \frac{d^{k} g(E)}{d\left(E / k_{\mathrm{B}} T\right)^{k}}\right|_{e=e_{d}}
$$

where $c_{k+1}$ are constant close to unity $\left(c_{1}=\right.$ Euler constant $0.577, c_{2}=0.989, c_{3}=0.907, c_{4}=0.981$, c5 also and then equal to 0.999$)$.

(3) Most interesting, the differentiation of $\mathrm{B}$ against $E_{d}\left(\frac{d B}{d E_{d}}=\mathrm{A}_{0} g E_{d}\right)$ yields the shape of the distribution function.

(4) As B is expressed with only one variable $\mathrm{B}\left(E_{d}\right)$. It exhibits the same shape as $\mathrm{B}(T)$ for given $t$ or $\mathrm{B}(\ln (t))$ for given $T$. Thus, we deduce that if $g(E)$ does not depend on $T$, isochrons and isotherms are equivalents:

For isotherms $\Delta n$ should be measured at the same temperature.

For isochronous annealing, there is a criterion in the temperature step and duration of the step (see Section 4.2).

For a given time, $[B](t, T)$ is the area under the dotted curve as in Figure 22. As $t$ increases, $x$ moves to the right hand side and more difficult pathways are used. 


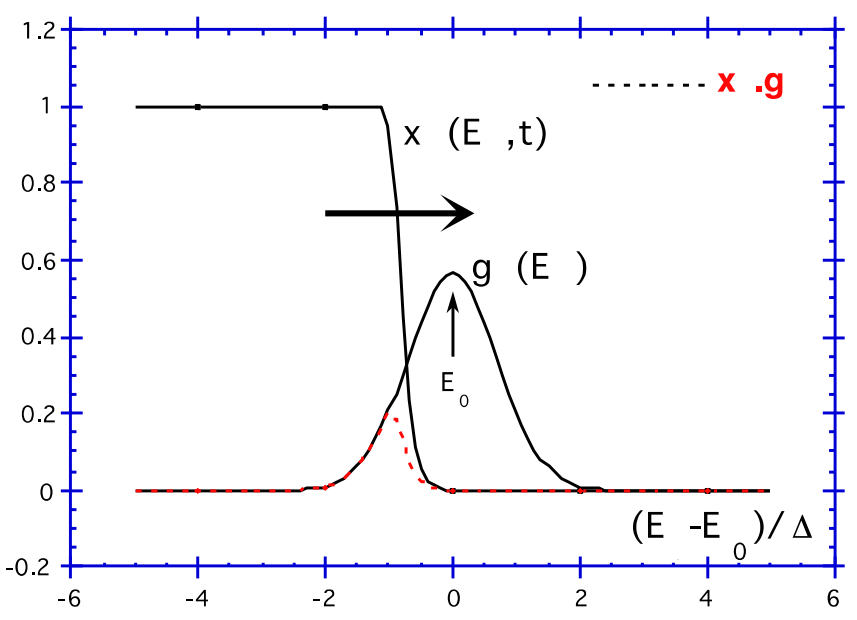

Figure 22. Progress of advancement degree along the distribution function.

2.2.3. Examples for Time Dependence Computation

(Step 1) Integration using a Gaussian distribution as the following:

$$
g(E)=\frac{1}{\Delta \sqrt{\pi}} \exp \left(-\left(\frac{E-E_{0}}{\Delta}\right)^{2}\right) \text { with } E_{0} \geq 2 \Delta \text { centered on } E_{0} \text {; width } \Delta
$$

yields:

$$
\begin{aligned}
& {[B](t, T)=A_{0} \frac{1}{\Delta \sqrt{\pi}} \int_{0}^{E_{d}(t, T)} \exp \left(-\left(\frac{E-E_{0}}{\Delta}\right)^{2}\right) d E} \\
& {[B](t, T)=A_{0} / 2\left[1-f_{\text {err }}\left(\frac{E_{d}-E_{0}}{\Delta}\right)\right], \text { where } f_{\text {err }} \text { is the error function }}
\end{aligned}
$$

(Step 2) The integration of a sigmoid differentiation distribution:

$$
g(E)=\frac{1}{k_{B} T_{0}} \frac{\exp \left(\frac{E-E_{0}}{k_{B} T_{0}}\right)}{\left(1+\exp \left(\frac{E-E_{0}}{k_{B} T_{0}}\right)\right)^{2}}
$$

yields:

$$
[B](t, T)=A_{0} \int_{0}^{E_{d}(t, T)} g(E) d E=A_{0} \frac{\exp \left(\frac{E_{d}(t, T)-E_{0}}{k_{B} T_{0}}\right)}{1+\exp \left(\frac{E_{d}(t, T)-E_{0}}{k_{B} T_{0}}\right)}=A_{0} \frac{\left(k_{0} t\right)^{\frac{T}{T_{0}}} \exp \left(-\frac{E_{0}}{k_{B} T_{0}}\right)}{1+\left(k_{0} t\right)^{\frac{T}{T_{0}}} \exp \left(-\frac{E_{0}}{k_{B} T_{0}}\right)}
$$

(Step 3) with a Poisson distribution: 


$$
g(E)=\frac{1}{k_{B} T_{0}} \exp \left(-\frac{E}{k_{B} T_{0}}\right)
$$

We obtain:

$$
[B](t, T)=A_{0} \int_{0}^{E_{d}(t, T)} g(E) d E=A_{0}\left[1-\exp \left(-\frac{E_{d}(t, T)}{k_{B} T_{0}}\right)\right]=A_{0}\left[1-\left(k_{0} t\right)^{-\frac{T}{T_{0}}}\right] \text { and } T<T_{0}
$$

\subsubsection{How to Obtain $k_{0}$ and $g(E)$ (Figure 23)}

(1) Measurement of several isochrons or isotherms writing kinetics and replotting against a new abscissa;

(2) $k_{0}$ fitting for curve collapsing and;

(3) Differentiation $\left.\frac{\partial[B]\left(E_{d}, T\right)}{\partial E_{d}}\right)_{T}=A_{0} g_{1}\left(E_{d}\right)$.

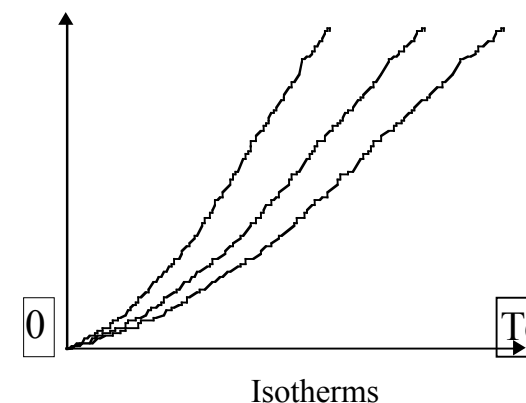

Temperature
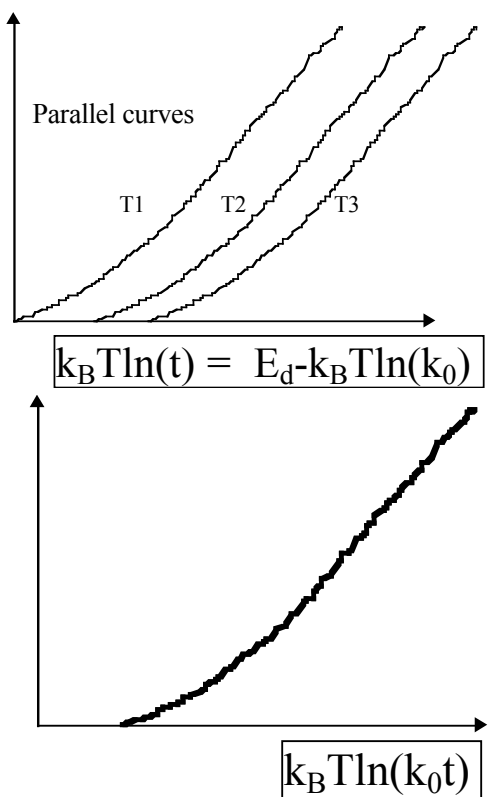

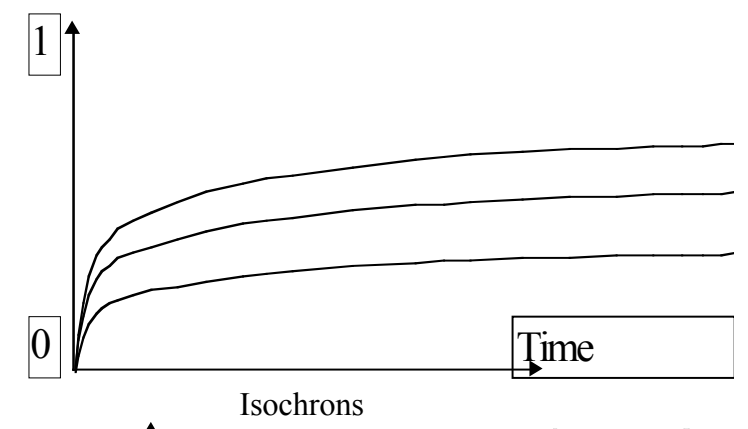

Isochrons
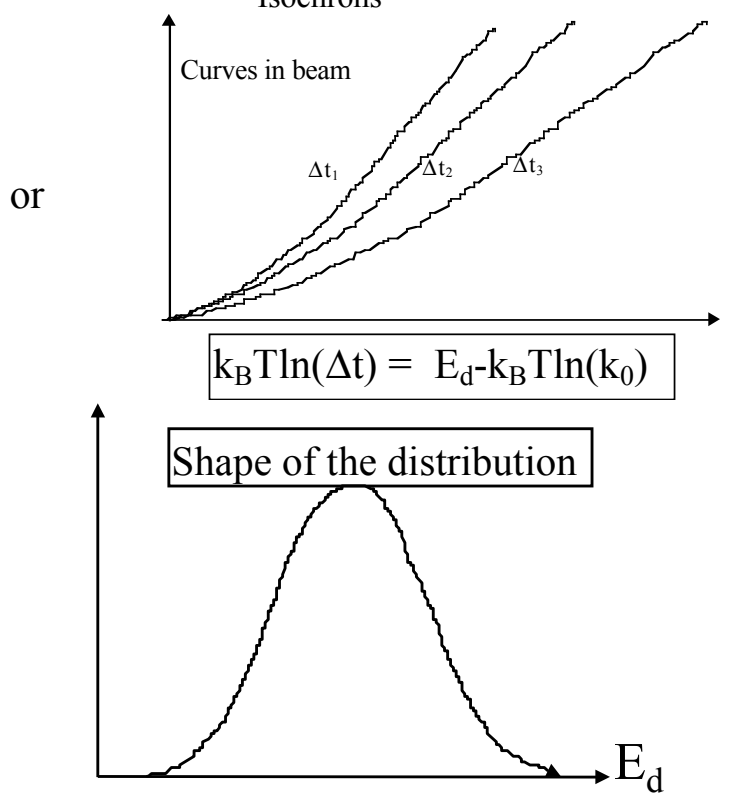

Figure 23. Procedure to obtain $k_{0}$ and $g(E)$.

We can note that $k_{0}$ and $g(E)$ are determined separately. The first one is related to the order of the kinetics and the second one is a measurement of the disorder of the glass. 


\subsection{Kinetics of Erasing}

The hypotheses for modeling the relaxation behavior, i.e., the erasure $(\mathrm{A} \leftarrow \mathrm{B})$, are basically the same as for the forward reaction $(\mathrm{A} \rightarrow \mathrm{B})$. There is a symmetry in the approach. The only difference is that the starting population is different. Whereas all sites are under A form for a forward reaction, it may be under A or B form for relaxation, i.e., the writing has not transformed all A content, but only the easiest part (non-saturated writing). When the writing of the kinetics is completed, or is not distributed, the same computation as Section 2.2 applies in replacing A by B and vice versa, and, considering that the activation energy is for the backward reaction, the distribution is different (see below).

Otherwise, it is necessary to consider a starting distribution for $\mathrm{B}$ that is equal to $A_{0} x_{+}\left(E_{+}, t_{w}, T_{w}\right) g_{+}\left(E_{+}\right)=B_{0}\left(E_{+}, t_{w}, T_{w}\right)$, i.e., the advancement degree times the distribution function for writing the reaction with $t_{w}, T_{w}$ the time and temperature of writing. We have to consider the following expression for B content, at time $t$, and at temperature $T$ when forward pathways are independent of backward pathways.

$$
[B](t, T)=A_{0} \int_{0}^{\infty} \int_{0}^{\infty} x_{+}\left(E_{+}, t_{w}, T_{w}\right)\left(1-x_{-}\left(E_{-}, t, T\right)\right) g_{+}\left(E_{+}\right) g_{-}\left(E_{-}\right) d E_{-} d E_{+}
$$

This is equivalent to:

$$
[B](t, T)=\underbrace{A_{0} \int_{0}^{\infty} x_{+}\left(E_{+}, t_{w}, T_{w}\right) g_{+}\left(E_{+}\right) d E_{+}}_{B_{0}\left(t_{w} T_{w}\right)} \int_{0}^{\infty}\left(1-x_{-}\left(E_{-}, t, T\right)\right) g_{-}\left(E_{-}\right) d E_{-}
$$

Therefore, the same approach as Section 2.2 can be used again for solving this integral. Otherwise, when forward and backward reactions use the same pathways, the energy of the reverse reaction can be related to energy of the forward reaction and $B_{0}\left(E_{+}\right)$becomes $B^{\prime}{ }_{0}\left(E_{-}\right)$.

Let us now recall the methods of computation that are applicable to all cases.

\subsubsection{First Group of Assumptions}

(1) One elementary reaction $\mathrm{A} \stackrel{k}{\longleftarrow} \mathrm{B}$ (limiting process), $\mathrm{A} \rightarrow \mathrm{B}$ is the writing process;

(2) Thermal activation, $E_{-}, k_{-}\left(E_{-}\right)=k_{-}^{0} \exp \left(-E / k_{B} T\right)$;

(3) $E$ - is distributed, i.e.,

- variable chemical pathways;

- the saddle point or the B state are sensitive to various structural configurations as decrived in Figure 24;

- $g(E)$ is the distribution function for backward reaction, it can be Gaussian for a thermal disorder or Poisson for a diffusional disorder or differentiation of a sigmoid, or also top hat function.

The total B content can be expressed as:

$$
[B](t, T)=\int_{0}^{\infty} B_{0}\left(E_{-}\right)\left[1-x_{-}\left(E_{-}, t, T\right)\right] g_{-}\left(E_{-}\right) d E_{-}
$$


$B_{0}(E)$ is the initial distribution of occupied sites according to the activation energy, regardless of the distribution functions, independent or not. $1-x$ is the degree of retardation.

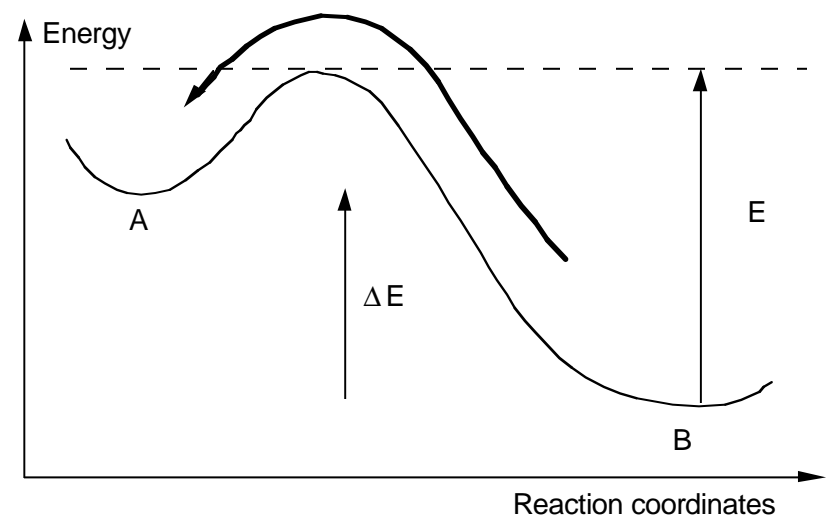

Figure 24. Reaction pathway for reverse reaction.

\subsubsection{Second Group of Assumptions (towards the Computation of Time Dependence)}

For computing the time and temperature dependences of the B content $([B](t, T)$, we have to make the following assumptions:

(1) Most of the elementary reactions in solids are first order; some are second order, when two species associate, but these are much less likely. Thus, this is not so severe and we can write:

$\mathrm{A} \stackrel{k}{\longleftarrow} \mathrm{B}, \frac{d x_{-}}{d t}=k_{-}\left(1-x_{-}\right), x_{-}\left(E_{-}, t, T\right)=1-\exp \left(-k_{-}\left(E_{-}, T\right) t\right)$ is the advancement degree of the reaction.

(2) Concept of demarcation energy, $E_{d}$

$x_{-}\left(E_{-}, t, T\right)$ is a function that varies very quickly with $E . B_{0}(E)$ is the initial population, produced by the FBG writing.

$$
x_{-}\left(E_{-}, t, T\right)\left\{\begin{array}{rr}
=1 & \text { for low energies (reaction completed) } \\
=0 & \text { for high energies (reaction did not begin) }
\end{array}\right.
$$

When $x_{-}\left(E_{-}, t, T\right)$ varies faster than $g_{-}\left(E_{-}\right)$, it can be approximated to a Heaviside function at energy $E_{d}^{-}$and, thus, we can read:

$$
\left.[B]_{T}(t, T)=\int_{0}^{\infty} B_{0}\left(E_{-}\right) \exp \left(-k_{-}\left(E_{-}, T\right) t\right) g_{-}\left(E_{-}\right)\right) d E_{-}=\int_{E_{d}^{-}}^{\infty} B_{0}\left(E_{-}\right) g_{-}\left(E_{-}\right) d E_{-}
$$

$E_{d}$ is properly defined by $\left.\frac{\partial^{2} x_{-}}{\partial E^{2}}\right)_{t, T}\left(E=E_{d}\right)=0$. For a first order reaction, $E_{d}^{-}=k_{B} T \ln \left(k_{-}^{0} t\right)$. The reaction properties (i.e., $k_{-}^{0}$ ) are only appearing in $E_{d}$, the disorder (relevant of the glass) appears in $g_{-}\left(E_{-}\right)$. Finally, $[B](t, T)$ is the area under the red dotted curve in Figure 25. The retardation degree $1-x-(E, t, T)$ in the progression erases the $\mathrm{B}$ distribution. 


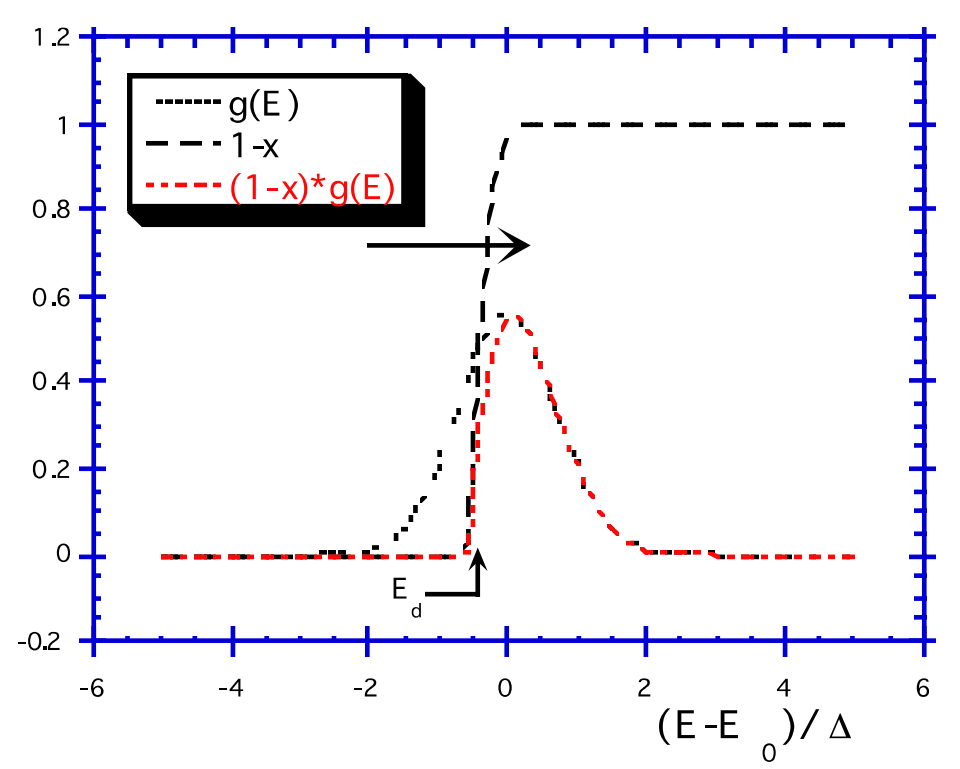

Figure 25. Reaction progresses by removing $\mathrm{B}$ sites, from less stable to the most stable sites.

- Some Consequences of the Theory:

(1) $[B](t, T)$ can be expressed as a function of the unique variable called demarcation energy $\left(E_{d}^{-}\right)$. The curve $[B]\left(E_{d}^{-}\right)$is called the master curve (MC), as it is unique, regardless of what the $(t, T)$ couple may be for a given value of $E_{d}$. We can note that $T$ is equivalent to $\ln t$ in $E_{d}^{-}$and, thus, isochronal ageing data are equivalent to isothermal ageing data for establishing MC. Note that from a practical point of view, most people consider that $\operatorname{NICC}\left(E_{d}^{-}\right)$is the master curve. Now, the $\mathrm{MC}$ plot allows the user to predict the grating lifetime, providing that the anticipated conditions of BG (i.e., $E_{d}^{-}=f\left(t_{\text {use, }} T_{\text {use }}\right)$ correspond to a point on the MC that has been actually sampled during the annealing experiment.

(2) Notice also that the distribution function is included in the MC differentiation as: $\frac{d[B]}{d E_{d}^{-}}=-B_{0}\left(E_{d}^{-}\right) g_{-}\left(E_{d}^{-}\right)$.

(3) Thermal stability increases along with the ageing time (hardening). This is due to the fact that the less stable sites are removed along the ageing.

- (1) When the pathways are the same in both senses, the distribution function is the same for forward and backward reactions, as shown in the scheme below. If the writing kinetics are not saturated, the forward distribution function is not saturated and, thus, neither is the backward distribution. The stability is dependent on the writing (time and power density that is included in $k_{0}^{+}$, see $B_{0}\left(E_{d}\right)$ in definition) 


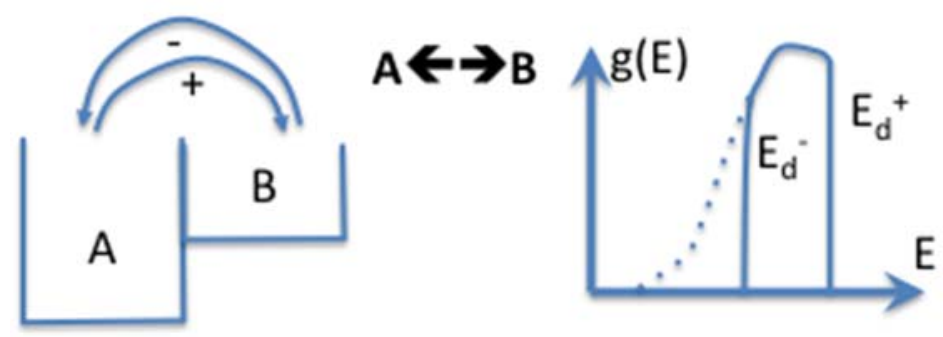

- (2) When there is no correlation between the distribution functions because the pathways are not the same, there are two independent distribution functions, as shown in the scheme below. The B sites are randomly occupied whatever its stability. The complete distribution function of the backward reaction has to be used. The stability is independent of writing (i.e., on the initial grating strength), see Section 2.3.3.
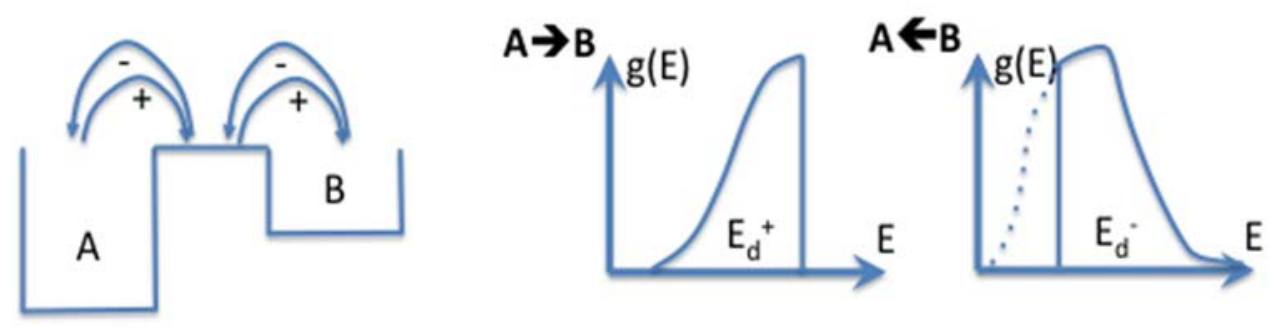

2.3.3. A Few Examples of Time Dependence Computations Assuming $B_{0}(E)=B_{0}$

If $g_{-}\left(E_{-}\right)$is Gaussian then:

$$
[B](t, T)=B_{0}\left(\frac{1-f_{\text {err }}\left(\frac{k_{B} T \ln \left(k_{+}^{0} t\right)-E_{0}}{\Delta}\right)}{2}\right) \approx \underbrace{B_{0}\left(1-a\left(k_{+}^{0} t\right)^{\frac{k_{B} T}{\Delta}}\right)}_{\text {for low } t}
$$

If $g_{-}\left(E_{-}\right)$is a Poisson distribution then: $[B](t, T)=a\left(k_{+}^{0} t\right)^{-\frac{k_{B} T}{\Delta}} ; \Delta$ is the width of the distribution.

If $g_{-}\left(E_{-}\right)$is a differentiation of a sigmoid then: $[B](t, T)=B_{0}\left(\frac{1}{1+a\left(k_{+}^{0} t\right)^{\frac{3 k_{B} T}{\Delta}}}\right)$

If $g_{-}\left(E_{-}\right)$is a top-hat of width $\Delta$ then: $[B](t, T)=B_{0}\left(\frac{E_{\max }-k_{B} T \ln \left(k_{+}^{0} t\right)}{\Delta}\right)$, etc.

2.3.4. Incomplete forward Reaction in the Case of Dependent Backward and Forward Pathways $\left(B_{0}(E)\right)[36]$

If there is dependence between the writing and the relaxation, the distributions are the same but shifted in energy, it is, in this case, valuable to know the relationship between $E_{+}$and $E_{-}$. We can solve the problem rather easily when the forward and backward reactions use the same pathways. In this case, $E_{-}-E_{+}=\Delta E$ where $\Delta E$ is the formation energy difference between A and B (Figure 26). 


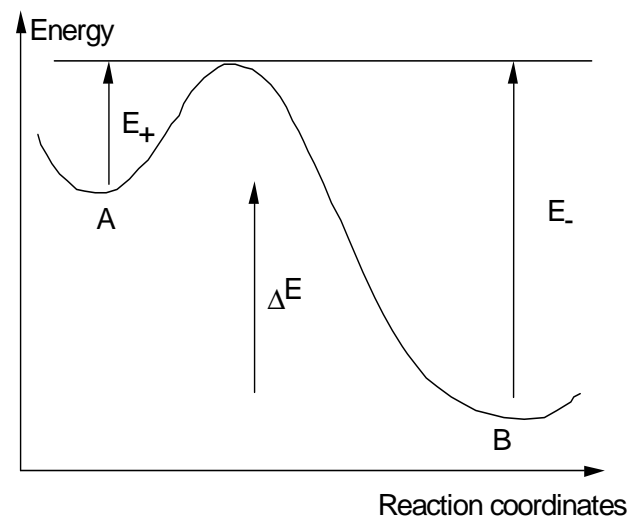

Figure 26. Relationship between activation energies.

We consider the initial distribution of $\mathrm{B}, B_{0}\left(E_{+}, t_{w}, T_{w}\right)=A_{0}\left[1-\exp \left(-k\left(E_{+}, T_{w}\right) t_{w}\right)\right]$ in the following integral $B(t, T)=\int_{0}^{\infty} B_{0}\left(E_{+}, t_{w}, T_{w}\right) \exp \left[-k_{-}\left(E_{-}, T\right)\right] g\left(E_{-}\right) d E$.

Hypothesis: the forward and backward pathways are the same $\geq E_{-}-E_{+}=\Delta E$

$$
A \stackrel{k_{-}}{\longleftarrow} B k_{-}=k_{-}^{0} \exp \left(-\frac{E_{-}}{k_{B} T}\right)
$$

For each $E_{-}, x_{-}\left(E_{-}, t\right)=1-\exp \left[-k_{-}\left(E_{-}\right) t\right]$.

$$
B(t, T)=A_{0} \int_{0}^{\infty} \underbrace{\left[1-\exp \left(-k_{+}\left(E_{+}, T_{w}\right) t_{w}\right.\right.}_{\text {define } E_{d}^{+}} \underbrace{\exp \left(-k_{-}\left(E_{-}, T\right)\right)}_{\text {define } E_{d}} g_{-}\left(E_{-}\right) d E_{-}
$$

in the scale of $E_{-}$, we define $E_{d}^{++}=E_{d}^{+}+\Delta E$, considering that each $E_{+}$is related to an $E_{-}$, which is $E_{+}+\Delta E$. This means that the function $1-\exp (-k+t)$ switching at $E^{+} d$ in the $E_{+}$scale commutes at $E^{+}{ }_{d}+\Delta E$ in the scale of $E_{-}$, i.e., there is no more $\mathrm{B}$ above this value or there is no reverse reaction above $E_{d}^{+}+\Delta E$ (Figure 27), thus $B(t, T)=A_{0} \int_{E_{d}^{-}}^{E_{d}^{+}+\Delta E} g_{-}\left(E_{-}\right) d E_{-}=A_{0} \int_{E_{d}^{-}-\Delta E}^{E_{d}^{+}} g_{+}\left(E_{+}\right) d E_{+}$.

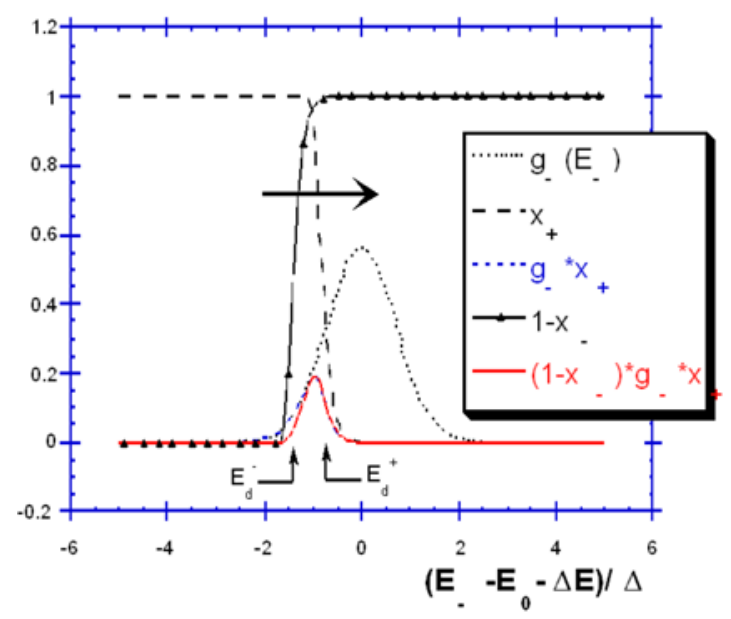

Figure 27. Erasure of incomplete distribution. 
We can note that the stability increases with an increase of $t_{w}$ or $T_{w}$ because $E^{+} d$ increases, leading to the occupation of more stable sites.

\subsubsection{Gaussian Distribution}

$$
\begin{gathered}
g_{+}\left(E_{+}\right)=\frac{1}{\Delta \sqrt{\pi}} \exp \left(-\left(\frac{E_{+}-E_{0}}{\Delta}\right)^{2}\right) \text { with } E_{0} \geq 2 \Delta \\
{[B](t, T)=A_{0} \int_{E_{d}^{+}=k_{B} T_{w} \ln \left(k_{+}^{0} t_{w}\right)}^{E_{d}^{-}-\Delta E=k_{B} T \ln \left(k_{-}^{0} t\right)-\Delta E} g_{+}\left(E_{+}\right) d E_{+}} \\
=A_{0}\left[1+f_{\text {err }}\left(\frac{E_{+}-E_{0}}{\Delta}\right)\right]_{k_{B} T \ln \left(k_{-}^{0} t\right)-\Delta E}^{k_{B} T_{w} \ln \left(k_{+}^{0} t_{w}\right)}
\end{gathered}
$$

defining $\eta\left(t_{w}, T_{w}\right)$ as the proportion to saturation by $\eta=\frac{1+f_{\text {err }}\left(\frac{k_{B} T_{w} \ln \left(k_{+}^{0} t_{w}\right)-E_{0}}{\Delta}\right)}{2}$ and $B_{0}=A 0 \eta$.

$$
\text { we get }[B](t, T)=B_{0}\left[\frac{2 \eta-1-f_{\text {err }}\left(\frac{k_{B} T \ln \left(k_{-}^{0} t\right)-\Delta E-E_{0}}{\Delta}\right)}{2 \eta}\right\rceil
$$

Isochrons $\mathrm{B}(T)$ curves are plotted for several values of $\eta$ in Figure 28.

We find that as $\eta$ increases on $t_{w}$ and $T_{w}, \frac{[B]}{B_{0}}$ increases as well and, thus, the Bragg gratings thermal stability increases. This arises from the fact that high activation energy pathways are used or more stable sites are produced. In a few cases, this will have a real importance on the bandwidth stability of Bragg gratings (see Section 4). After annealing, the easiest pathways are removed, the most difficult ones remain, and, thus, the stability is increased (hardening).

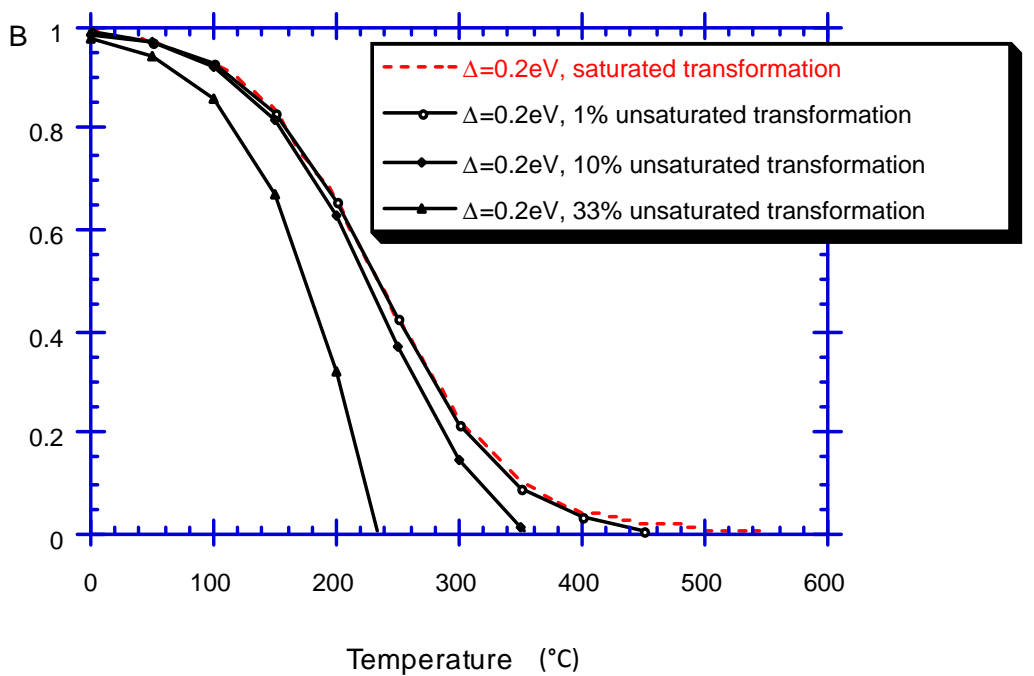

Figure 28. The unsaturated writing leads, in this case, to disymmetrical isochronal curves. 
2.3.4.2. Sigmoid Differentiation Distribution

$$
g_{+}\left(E_{+}\right)=\frac{1}{k_{B} T_{0}} \frac{\exp \left(\frac{E_{+}-E_{0}}{k_{B} T_{0}}\right)}{\left[1+\exp \left(\frac{E_{+}-E_{0}}{k_{B} T_{0}}\right)\right]^{2}}
$$

When the forward reaction is completed, the erasure follows the equation below:

$$
[B](t, T)=\frac{A_{0}}{1+\left(k_{-}^{0} t\right)^{\frac{T}{T_{0}}} \exp \left(-\frac{\Delta E+E_{0}}{k_{B} T_{0}}\right)}
$$

However, when it is not, $t_{w}$ and $T_{w}$ appear in the equation.

$$
\frac{[B](t, T)}{B_{0}}=\frac{1-\left(k_{-}^{0} t\right)^{\frac{T}{T_{0}}} \exp \left(-\frac{\Delta E}{k_{B} T_{0}}\right)}{1+\left(k_{-}^{0} t\right)^{\frac{T}{T_{0}}} \exp \left(-\frac{\Delta E+E_{0}}{k_{B} T_{0}}\right)}
$$

Defining $\eta=\frac{1}{1+\left(k_{+}^{0} t_{w}\right)^{\frac{T_{w}}{T_{0}}}}$ as the proportion to saturation, we get $\frac{[B](t, T)}{B_{0}}=\frac{1-\left(k_{-}^{0} t\right)^{\frac{T}{T_{0}}} \exp \left(-\frac{E_{0}+\Delta E}{k_{B} T_{0}}\right) \frac{1-\eta}{\eta}}{1+\left(k_{-}^{0} t\right)^{\frac{T}{T_{0}}} \exp \left(-\frac{E_{0}+\Delta E}{k_{B} T_{0}}\right)}$.

2.3.4.3. Poisson Distribution

$$
\begin{aligned}
& g_{+}(E)=\frac{1}{k_{B} T_{0}} \exp \left(\frac{-E_{+}}{k_{B} T_{0}}\right)
\end{aligned}
$$

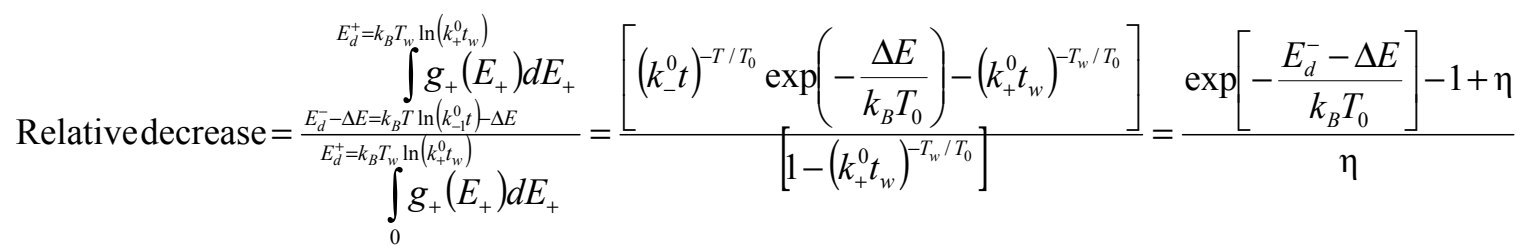

\section{Basic Experimental Analysis of the Erasure}

\subsection{Distribution Function Determination for the Reverse Reaction}

For finding the distribution function when there are no ideas of the shape, one has to follow the same method as in Section 2.2. 
(1) note: $\frac{d[B]}{d E_{d}^{-}}=-B_{0}\left(E_{d}^{-}\right) g_{-}\left(E_{d}^{-}\right)$

(2) with the assumptions 2.3.1 and 2.3.2 (and even less, i.e., assumption on first order reaction is not necessary).

$$
[B](t, T)=\int_{0}^{\infty}\left(1-x_{-}\left(E_{-}-E_{d}^{-}\right)\right) B_{0}\left(E_{-}\right) g_{-}\left(E_{-}\right) d E_{-} \text {with } E_{d}^{-}=k_{B} T \ln \left(k_{0} t\right)
$$

We note that $t, T$ are equivalent $\geq$ equivalence between isotherms and isochrones.

The main problem is to find $k_{0}$. This is described here in Figure 29.

(1) Measurement of several isochrons or isotherms:

(2) $k_{0}$ fitting for curve collapsing. We adjust the constant $k_{0}$ for obtaining the collapse of the curves like in the Figure 29.

(3) Differentiation of the previous curve. $\frac{d[B]}{d E_{d}^{-}}=-B_{0}\left(E_{d}^{-}\right) g_{-}\left(E_{d}^{-}\right)$.
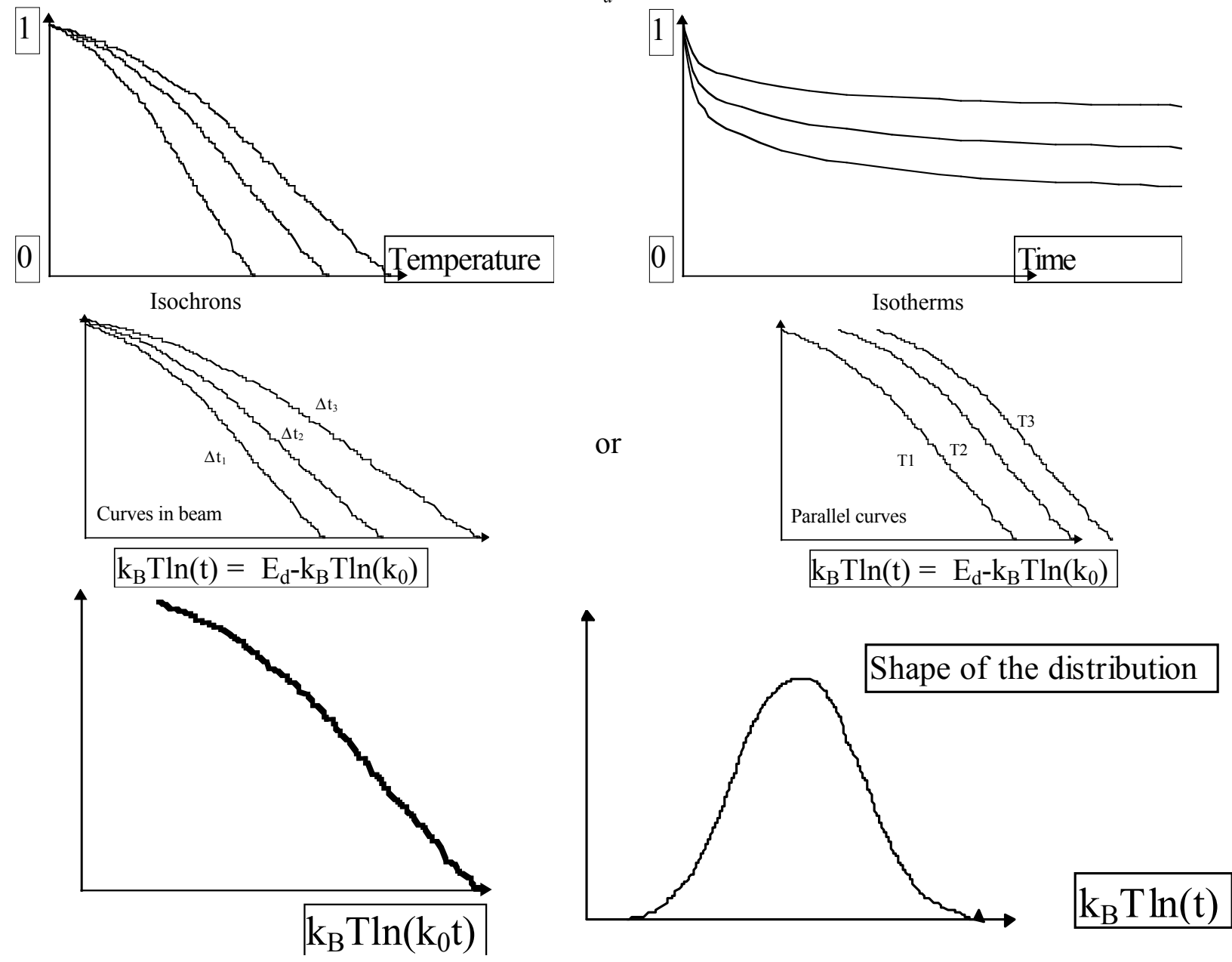

Figure 29. Procedure to obtain $k_{0}$ and $g(E)$. 


\subsection{Practice (or application of the theory)}

The example we have chosen is the case of Bragg grating, written in a tin doped optical fiber core. Here, we make the assumption that the refractive index change modulation is proportional to point index change (see Section 4). Results are from Razafimahatratra [37]. Five isotherms are available, which we can see in Figures 30 and $31\left(79,194,299,386,500^{\circ} \mathrm{C}\right)$. The last isotherm (Figure 31) is not recorded in the same conditions as the others (index change at the origin is $2.4 \times 10^{-4}$ for $500{ }^{\circ} \mathrm{C}$, whereas it is 2 $\times 10^{-4}$ for the others) but we will see that it is not annoying due to renormalization.

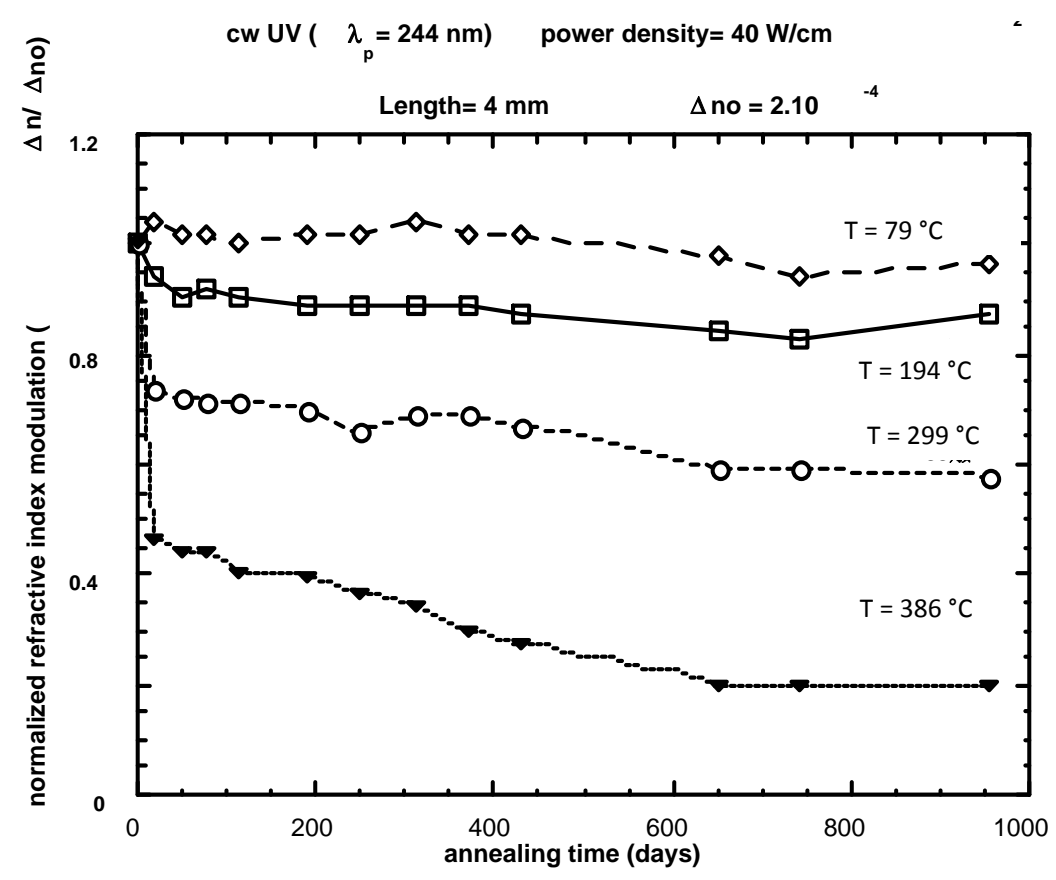

Figure 30. Isotherms from Razafimahatratra, reprinted with permission from [37]. Copyright 2000 University Lille 1.

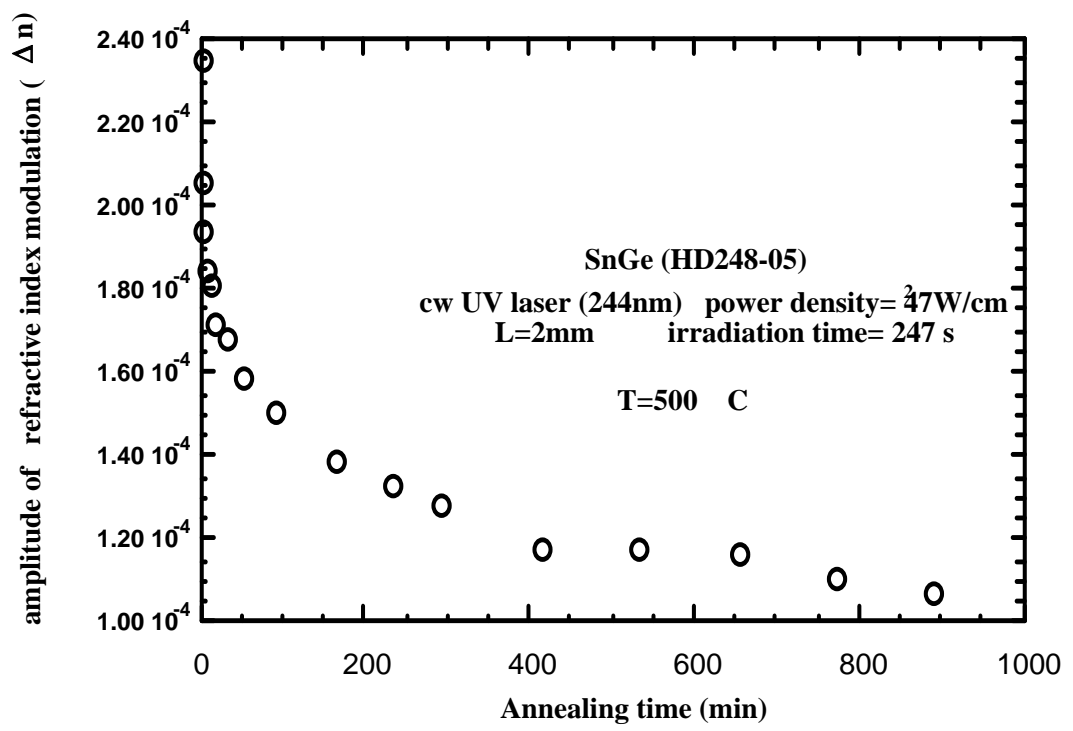

Figure 31. Isotherms from Razafimahatratra, reprinted with permission from [37]. Copyright 2000 University Lille 1. 
After plotting against $k_{\mathrm{B}} T \ln t$, we obtain Figure 32, which should show parts of parallel curves. We have to align these, looking for the constant $k_{0}$. This can be done automatically by fitting the polynomial of demarcation energy $k_{\mathrm{B}} T \ln \left(k_{0} t\right)$. This has been made in Figure 33, yielding $k_{0}=0.6$ to $2.4 \times 10^{12}$ day $^{-1}$. Here, we can check that it was possible to find a frequency factor, thus that the hypothesis of one order reaction for relaxation is fulfilled. Let us go on to distribution function determination. There are two solutions: one is to differentiate thefitted polynomial and try to fit a known expression, which is possible to find, for instance, in commercial software. Here, it seems realistic that the distribution is a bell shaped function, and, thus, an incomplete sigmoidal function in the case of identical forward and backward pathways (see Section 2.3.4.2). Figure 34 shows the fit in line with the expression below:

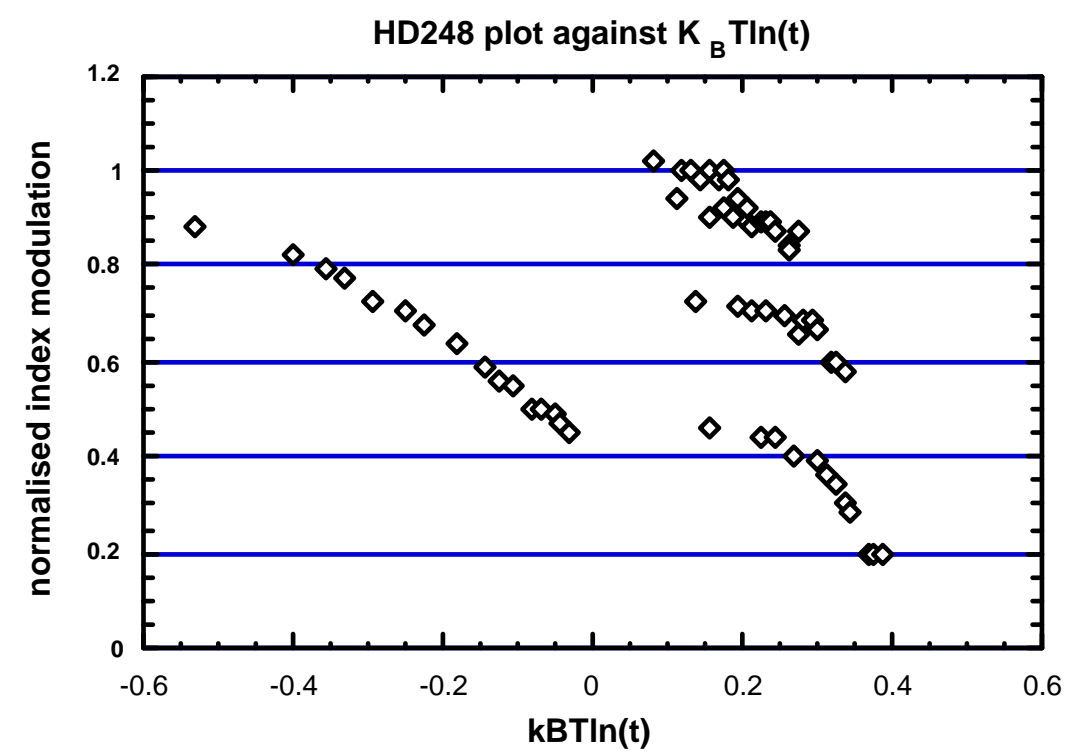

Figure 32. Plot of results of Figure 31 against $k_{\mathrm{B}} T \ln t$.

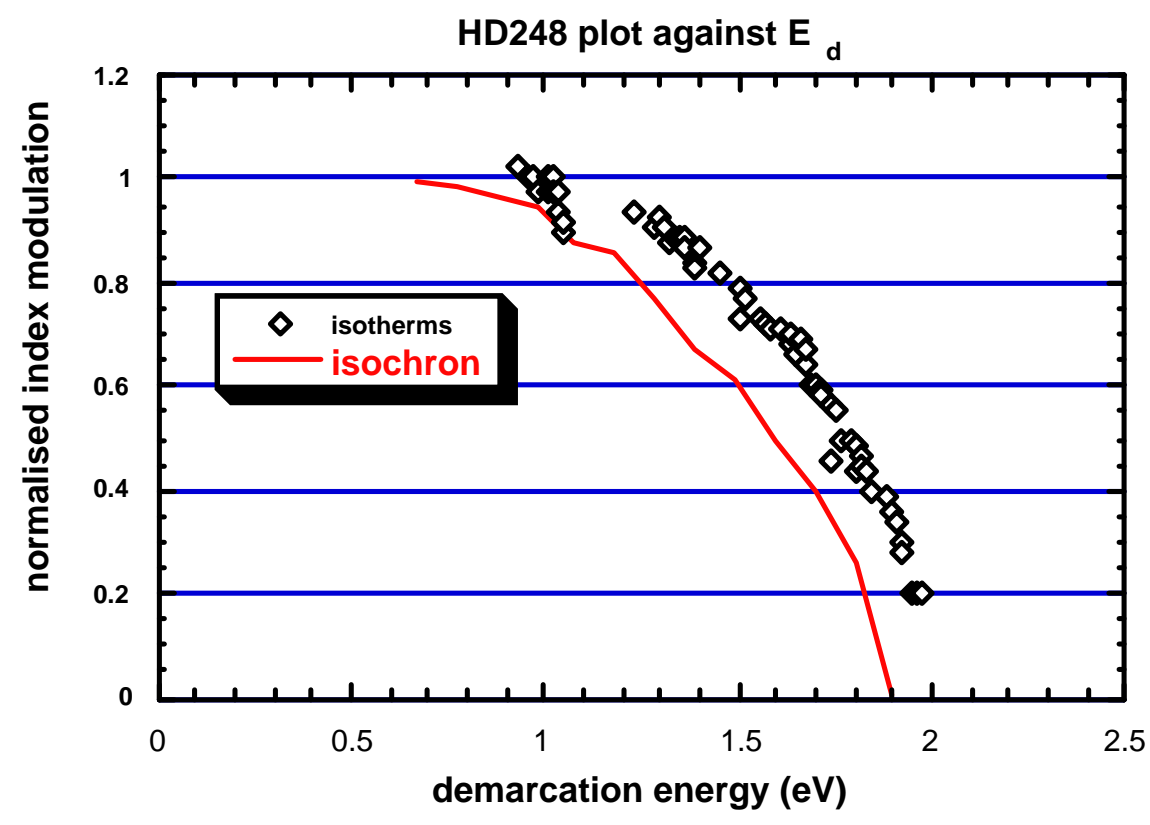

Figure 33. Plot against demarcation energy, an isochron has been added. 


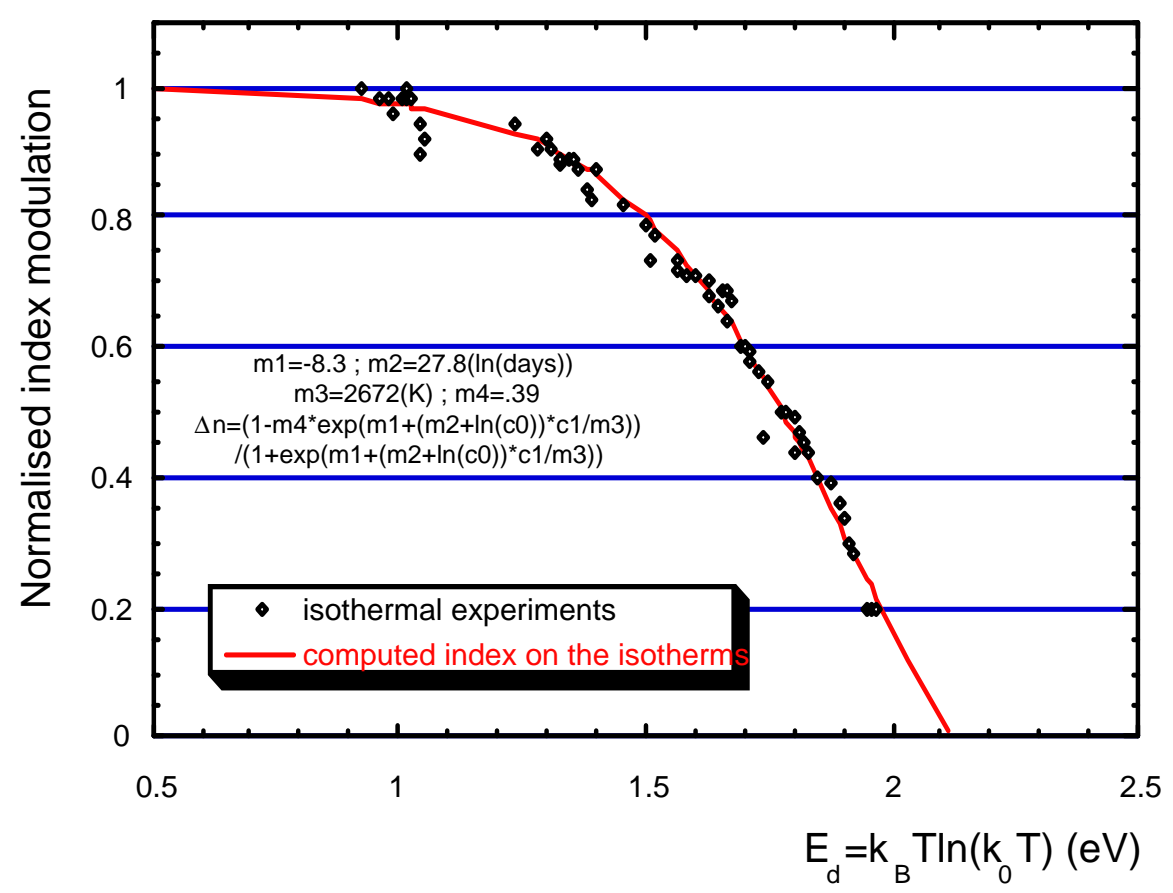

Figure 34. $\eta$ (the proportion to saturation of the gratings) is found at $72 \% \pm 10 \%$, the rate constant for the stability is $1.2 \times 10^{12}$ day $^{-1} \pm$ factor 2 , the pre-exponential factor is $2.5 \times 10^{-4} \pm 30 \%$ and $T_{0}=2670 \pm 200 \mathrm{~K}$.

$$
\frac{[B](t)}{B_{1}}=\frac{1-\left(k_{-}^{0} t\right)^{\frac{T}{T_{0}}} \exp \left(-\frac{E_{0}+\Delta E}{k_{B} T_{0}}\right) \frac{1-\eta}{\eta}}{1+\left(k_{-}^{0} t\right)^{\frac{T}{T_{0}}} \exp \left(-\frac{E_{0}+\Delta E}{k_{B} T_{0}}\right)}
$$

The gratings appear to be non-saturated, written to $72 \% \pm 10 \%$ in the sense that the index was not saturated (this has nothing to do with the reflectivity). The pre-exponential factor is $2.5 \times 10^{-4} \pm 30 \%$ and $T_{0}=2670 \pm 200 \mathrm{~K}$. As we have seen in Section 3.1, under the validity of a few assumptions, there is a $\ln t, \mathrm{~T}$ equivalence. The plot of an isochron in Figure 33 shows a shift that is explained by Niay et al. [37] by the different temperature of measurements of the reflectivity.

\subsection{Stabilization}

Since the life function is now available, it is possible that, for a maximum specified temperature of $T=273+40 \mathrm{~K}$ and 25 years, the grating strength will decrease to 0.974 . It is also possible to study the stabilization in order to eventually increase the lifetime of our grating. The principle is based on the distribution of B species stability at various sites. A burning-in process suppresses the less stable sites (see Figure 35). This cannot be achieved with a simple exponential kinetics (SIREPA). 


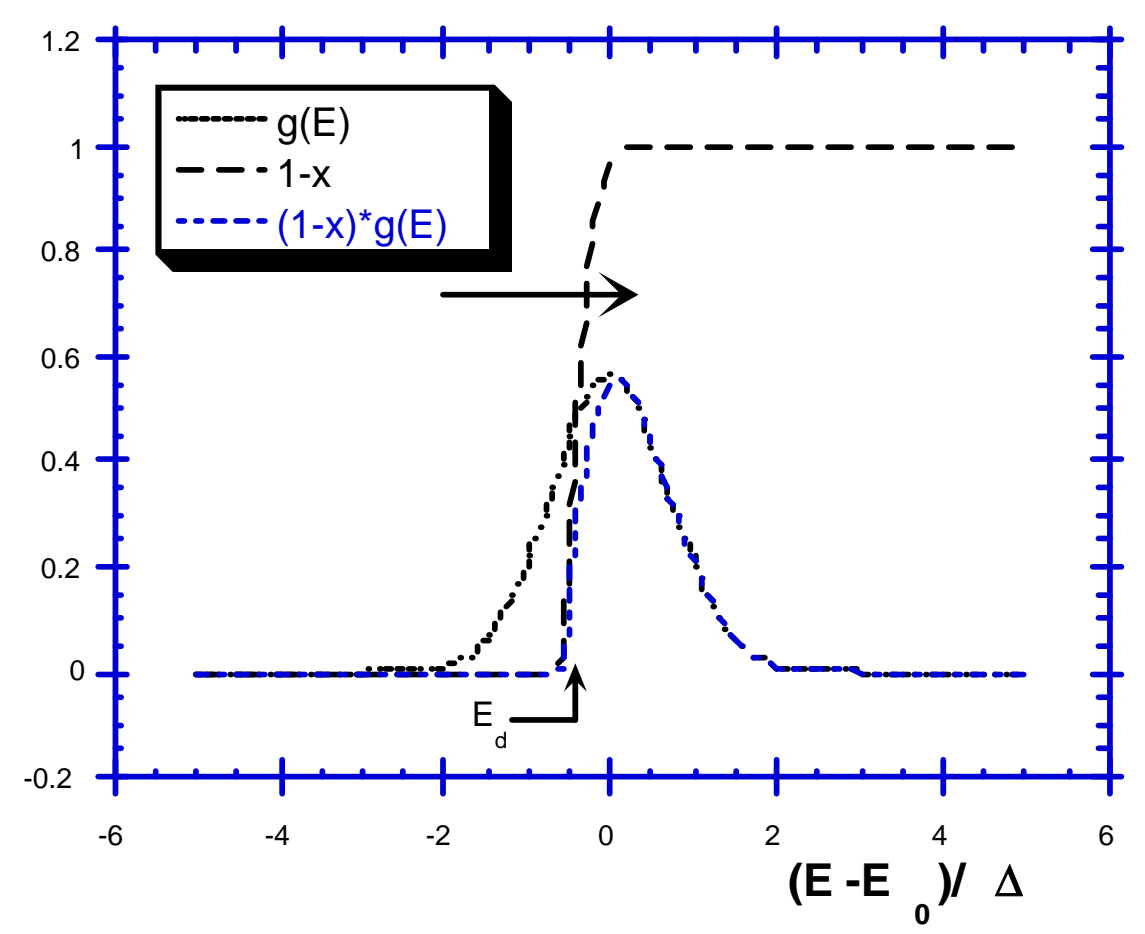

Figure 35. Effect of an annealing on B distribution: annealing will erase the less stable species.

This widespread method, used for increasing the stability of an FBG (Fiber Bragg Grating) after its inscription, consists in post-annealing the FBG for a time $t_{\text {anneal }}$ at a temperature $T_{\text {anneal }}>T_{\text {use }}$. A coherent rationale of this method is formulated from the above-mentioned approach: the treatment wipes out the portion of the index change that would normally decay over the lifetime of the device, and keeps only the stable portion of the index change. Notice that this method proves to be effective for enhancing the stability of index changes in either non-hydrogenated or $\mathrm{H}_{2}$-loaded Ge-doped fibers.

As shown in Figure 36, the stabilization (passivation) induced by an annealing can be computed by renormalizing the final value of the index change $\Delta n_{\mathrm{f}}$ after a time $t_{\mathrm{use}}$ at temperature $T_{\text {use }}$ by the index change $\Delta n_{1}$ after burning in process. If we impose that after a given time at a given temperature (let us say 25 years at $40{ }^{\circ} \mathrm{C}$ for instance), the lifetime ratio $\Delta n_{\mathrm{f}} / \Delta n_{1}$ has to remain within a given range $1-\varepsilon<\frac{\Delta n_{\mathrm{f}}}{\Delta n_{1}}<1$ with $\varepsilon<10 \%$, as an example, we obtain the passivation parameters (time $t_{\mathrm{a}}$ and temperature $T_{\mathrm{a}}$ ). Notice that, in general, we estimate the uncertainty on $k_{0}$ based on the data uncertainty, then we chose the worst case value and we added an extra safety margin (typically $\varepsilon<1 \%$ instead of $10 \%$ ). A thorough analysis is shown in [38].

From practical point of view and as shown in Figure 36, the stabilization (passivation) is usually defined by a passivation ratio, noted as $\Delta n_{0} / \Delta n_{1}$, which is defined by the FBG index modulation $\Delta n_{1}$ (or its reflectivity) after a passivation time $t_{\mathrm{a}}$ at a given passivation temperature $T_{\mathrm{a}}$ by the FBG index modulation $\Delta n 0$ (or its reflectivity) before the passivation process. Thus, if the end user imposes that after a given time $t_{\text {use }}$ at a given temperature $T_{\text {use }}\left(25\right.$ years at $45^{\circ} \mathrm{C}$, for instance), the lifetime ratio $\Delta n_{\mathrm{f}} / \Delta n_{1}$ has to remain within a given range $1-\varepsilon<\frac{\Delta n_{\mathrm{f}}}{\Delta n_{1}}<1$ with $\varepsilon<1 \%$ for instance, we determined the related passivation ratio $\Delta n_{0} / \Delta n_{1}$, e.g., 2. This means that the passivated FBG at a chosen passivation 
temperature $T_{\text {a }}$ (e.g., $220{ }^{\circ} \mathrm{C}$ in our study) must be erased of about a factor of two to respect the above-mentioned lifetime criteria $\left(\varepsilon<1 \%\right.$ during 25 years at $\left.45^{\circ} \mathrm{C}\right)$. Using the data in Figure 34 , here we have, $33 \mathrm{~h}$ at $110^{\circ} \mathrm{C}$.

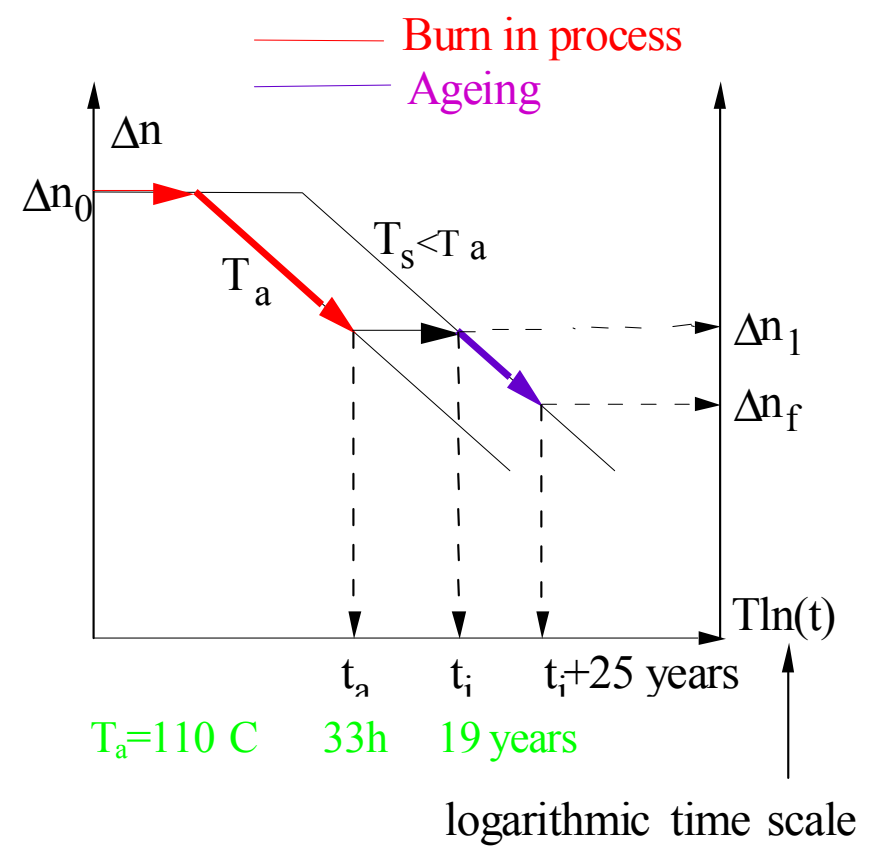

Figure 36. Scheme of the passivation process (also called burn-in process in this figure). $\Delta n_{0}$ is the FBG index modulation (or its reflectivity) before the passivation process at the temperature $T_{\mathrm{a}}$ for a given time $t_{\mathrm{a}} . \Delta n_{1}$ is the FBG index modulation after the passivation process. $\Delta n_{\mathrm{f}}$ is the final value of the index change after its life for a time $t_{\text {use }}$ at a given temperature $T_{\text {use. }}$

Important remark:

If the condition of aging of a system is reliable, the master curve of the passivated system can be deduced from the MC of the passivated system. Then, this new MC can be compared with an experimental one built from the passivated system. This works very well, but some precautions have to be taken when performing the comparison. More details can be found in pages 50-67 in [38]. Important points are as follows:

- $\quad$ the non-passivated master curve after annealing is truncated and needs to be renormalized.

- the origin of the time is usually taken from the beginning of the ageing after annealing and, thus, a time shift has to be applied on the previous demarcation energy. This is not negligible in the first $3 \%$ of ageing (see page 52 of [38]).

\section{Peculiar Properties in the Frame of Assumption Validity (Action of the Reversed Reaction)}

\subsection{Writing-Erasure Connection $(A \Rightarrow B, B \Rightarrow A)$}

We have seen that it is interesting to write at the highest temperature because $E_{d}$ increases on $T$ and, thus, also the stability (see Section 2.3.4). However, sometimes the reverse reaction $(B \Rightarrow A)$ becomes 
active, introducing a limitation. It cannot be neglected anymore regardless of whether the forward and backward pathways are the same or not.

For high $T$, equilibrium is developed regardless of if either the $g^{+}$or $g^{-}$distributions are dependent or not from each other because the reverse reaction can no longer be neglected.

(1) Case 1 (same pathways): we will show that the distribution function is cut at large $E_{d}$ at a more elevated temperature. Stability increases because more stable sites are filled [36] (see Section A below).

(2) Case 2 (different pathways, two distribution functions): stability increases because less stable sites are removed in the reverse distribution function during writing (Section B below).

\subsubsection{Writing in the Case of Reversible Reaction}

The equations applicable in the case of reversible kinetics are written below. Now, the degree of advancement $x+$ will never reach 1 , but will remain limited by the rate $k_{+}^{0} / k_{T}^{0}$.

$$
\begin{aligned}
& A \underset{k_{-}}{\stackrel{k_{+}}{\longrightarrow}} B x_{/}\left(E_{+}, t, T\right)=\frac{k_{+}^{0}}{k_{T}^{0}}\left(1-\exp \left(-k_{T} t\right)\right) \\
& \text { with } k_{T}^{0}(T)=k_{+}^{0}+k_{-}^{0} \exp \left(-\frac{\Delta E}{k_{B} T}\right)
\end{aligned}
$$

Using the same procedure as in Section 2.2.3, the integral of B is solved with a new expression for the demarcation energy, including the total rate constant in the $E_{+}$scale $\left(k_{T}^{0}(T), E_{D}^{+}=k_{B} T \ln \left(k_{T}^{0}(T) t\right)\right.$ (see [36]). Using a distribution from differentiation of a sigmoid, we get the following expression:

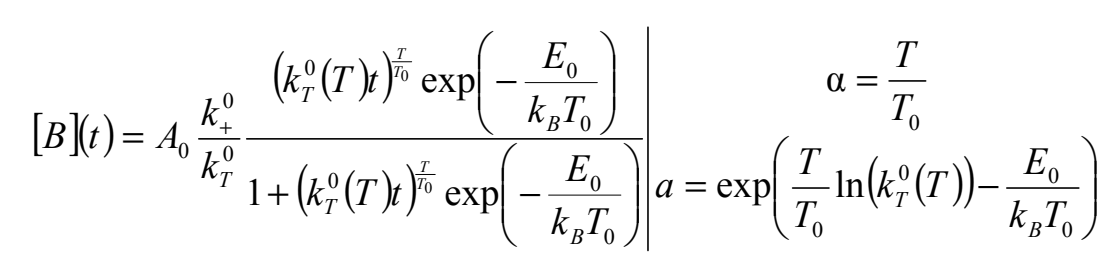

The expression of $[B](t, T)$ reveals that power law with the same exponent as in Section 2 is still followed, but the pre-exponential factor is modified. Now, the dependence of the pre-exponential factor a is super-exponential. This is due to the variation of total rate constant with temperature. The growth of index during writing will be faster at a higher temperature, but limited to $k_{1}^{0} / k_{T}^{0}$, which itself decreases with temperature. Thus, there is a compromise. $E_{d}$ is no more proportional to $T$ and, thus, it is no longer possible to find a $k_{0}$ with isochronous experiments. Two parameters are necessary $k_{+}^{0}$ and $k_{-}^{0}$. The equivalence between $\ln t$ and $T$ is broken.

Figure 37 is an example from P. Bernage et al. [4], where we can see that the reverse reaction becomes predominant at high temperature. 


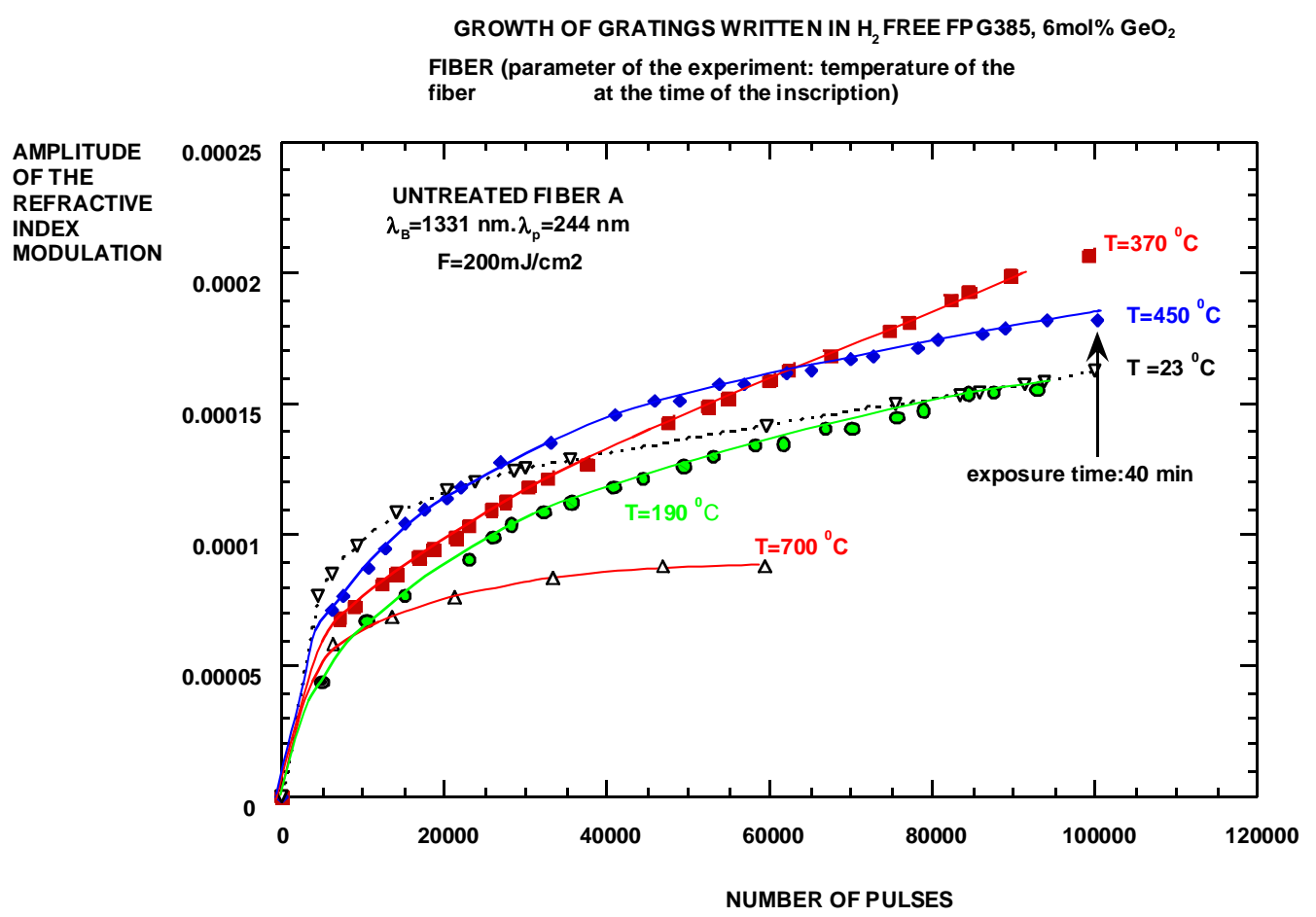

Figure 37. Writing kinetics results from Bernage et al., reprinted with permission from [4].

Copyright 1996 University Lille 1.

\subsubsection{Erasure in the Case of Reversible Reaction}

In this case, only the backward reaction $\mathrm{A} \stackrel{k_{-}}{-} \mathrm{B}$ is active because the forward rate is nulled when light power density is zero. The expression for $[B](t, T)$ is the same as in Section 2.3.4 replacing $k_{+}^{0}$ with $k_{T}^{0}$ in the corresponding expression for stability. Conclusions are, thus, the same as in the previous section, except that the temperature dependence is slightly more complex, as we can see in the formula below:

$$
\begin{gathered}
E_{D}^{+}=k_{B} T \ln \left(k_{T}^{0} T t\right) ; E_{D}^{-}=k_{B} T \ln \left(k_{-}^{0} T t\right) ; E_{-}-E_{+}=\Delta E \\
B_{0}\left(E_{+}, t_{w}, T_{w}\right)=A_{0} \frac{k_{+}^{0}}{k_{T}^{0}}\left[1-\exp \left(-k+\left(E_{+}, T_{w}\right) t_{w}\right] ;\right. \\
{[B](t, T)=\int_{0}^{0} B_{0}\left(E_{+}, t_{w}, T_{w}\right) \exp \left(-k_{-}\left(E_{-}, T\right) g_{-}\left(E_{-}\right) d E_{-}\right.} \\
\mathrm{A} \stackrel{k_{-}}{\mathrm{B}} k_{-}=k_{-}^{0} \exp \left(-\frac{E_{-}}{k_{B} T}\right) ; \\
{[B](t, T)=A_{0} \frac{k_{+}^{0}}{k_{T}^{0}} \int_{E_{d}^{+}+\Delta E}^{-} g_{-}\left(E_{-}\right) d E_{-}=\frac{k_{+}^{0}}{k_{T}^{0}} \int_{E_{d}^{-}-\Delta E}^{E_{+}^{+}} g_{+}\left(E_{+}\right) d E_{+}}
\end{gathered}
$$

\subsubsection{Stability When Forward and Backward Reactions Are Independent}

The results in Figure 38 are stability of Bragg gratings, written at different temperature in $\mathrm{H}_{2}$-loaded fibers. They are increasing as the writing temperature increases. The distribution function of the backward reaction is a top hat function since, in this isochronous plot, the curve is linear from 
ordinate (results above $800{ }^{\circ} \mathrm{C}$ are relevant of a second mechanism which is not presented in this paper. The distribution function for the forward reaction is probably a bell shape curve when a power law is detected, or more complex as two successive reactions are noted (see Section 5.3). Forward and backward distributions are independent as stability of gratings written with different strength, but at the same temperature, exhibit the same stability [39]. The expression is similar to the one in Section 2.3.3.

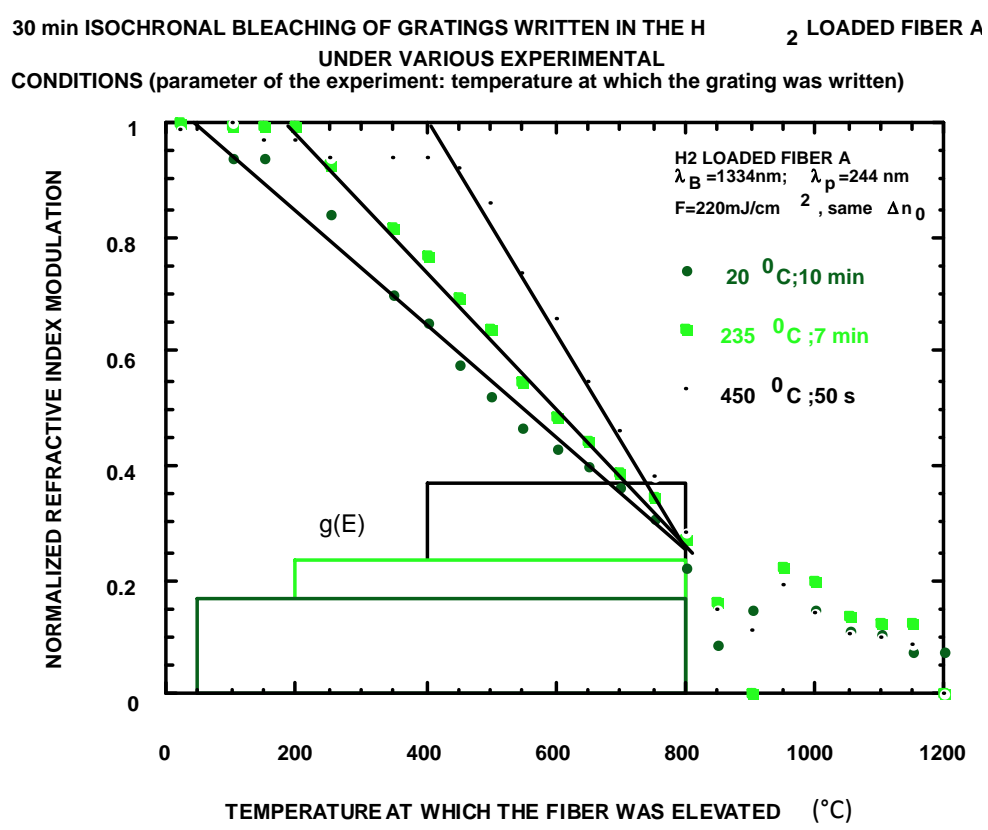

Figure 38. Stability of gratings written with the same $\Delta n_{0}$ but at a different temperature.

During writing at room temperature only reaction $\mathrm{A} \rightarrow \mathrm{B}$ is working, the square distribution lies from room $T$ to $800^{\circ} \mathrm{C}$. At a writing temperature of $235^{\circ} \mathrm{C}$, the chemical reaction needs less time to reach the same index change because the writing reaction is thermally activated. On the other hand, the reverse reaction is also thermally activated. This results in a partial bleaching of the species induced by the forward, i.e., the less stable sites. Therefore, the distribution function is "eaten" on the low energy side. As the distribution integral area should remain constant, its magnitude increases.

4.1.4. Writing in the Case of Independent Forward and Backward Reactions

The resolution in this case is not obvious. Let us observe the following equations:

$$
\begin{gathered}
A \underset{k_{-}}{\stackrel{k_{1^{+}}}{\leftrightarrows}} B x_{+}\left(E_{+}, E_{-}, T\right)=\frac{k_{+}}{k_{T}}\left(1-\exp \left(-k_{T} t\right)\right) \\
\text { with } k_{T}=k_{+}+k_{-} \\
B(t, T)=\int_{0}^{\infty} \int_{0}^{\infty} g_{+}\left(E_{+}\right) g_{-}\left(E_{-}\right) \frac{k_{+}\left(E_{+}\right)}{k_{T}\left(E_{+}, E_{-}\right)}\left(1-\exp \left(-k_{T}\left(E_{+}, E_{-}\right)\right)\right) d E_{+} d E_{-}
\end{gathered}
$$

The fact that there is no relation between $E_{+}$and $E_{-}$, and that there is $E_{+}$and $E_{-}$in $k_{T}$, renders the double integral unsplitable. As there is no simple solution for solving it, we will not go further in this direction. We can simply note that a limitation appears due to a reversible reaction. 


\subsection{Isotherm-Isochron Equivalence}

Before comparing isochrons and isotherms, it is necessary to be sure that the various measurements have been achieved at the same temperature, room temperature as an example. This means that the grating is quenched at room temperature after annealing at another temperature. This problem has been addressed extensively by Razafimahatratra et al. [40].

A second problem is that the isochrons steps have to be performed correctly, i.e., isochrons steps must be independent. This is achieved under the condition $\left(\delta t k_{0}\right)^{-\frac{\Delta T}{T_{\max }}}<<1$, where $\delta t$ is the duration of the annealing step, $\Delta T$ is the temperature-step increase, and $T_{\max }$ is the maximum temperature achieved on the isochrons. In that case, taking a new grating for each isochrons or using the same grating, step-by-step, are equivalent, as is shown in Figure 39a-d.

From the simple theory, $\Delta n=f\left(E_{d}\right), E_{d}=k_{\mathrm{B}} T \ln \left(k_{0} t\right)<=>(\ln t, T)$ equivalence.

Relevant assumptions:

- the distribution function is not $T$ dependent,

- $\quad$ only one limiting reaction.

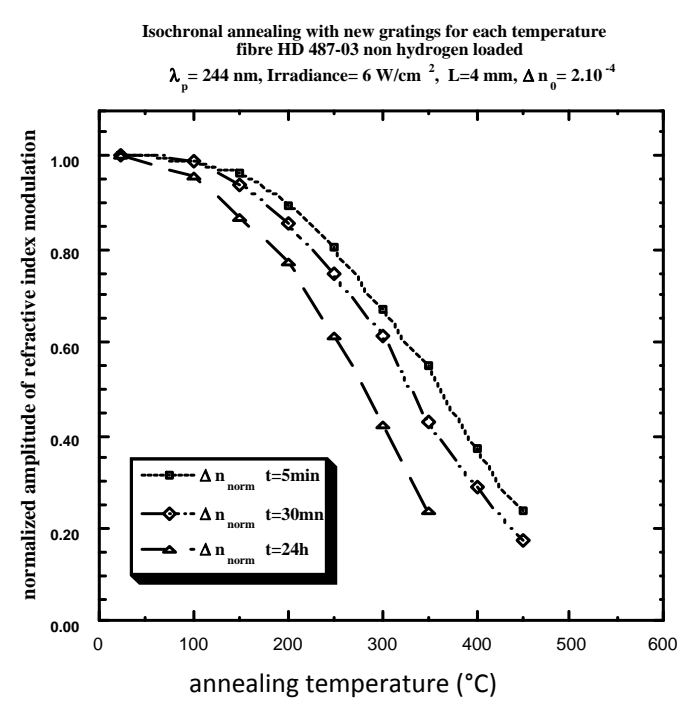

(a)

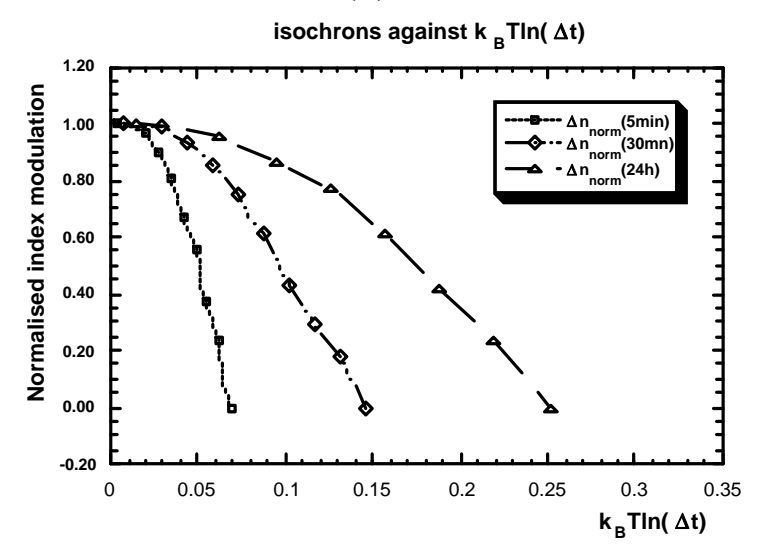

(c)

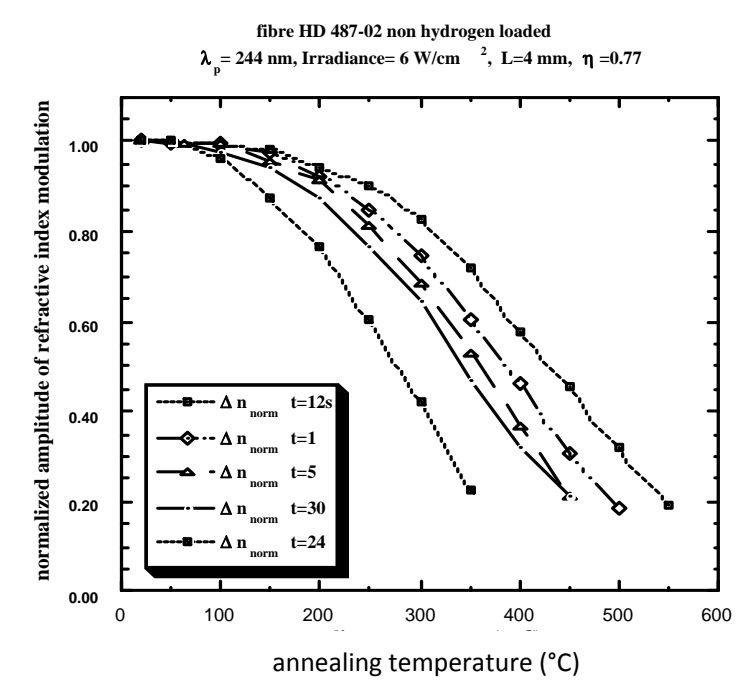

(b)

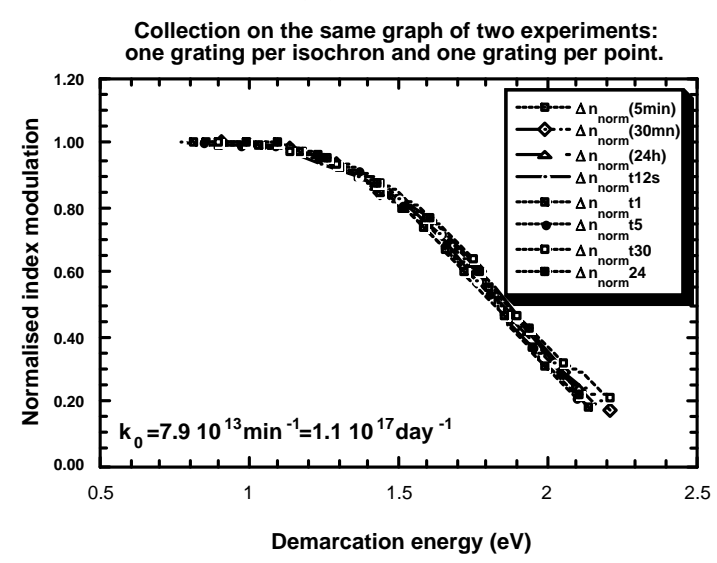

(d)

Figure 39. Cont. 


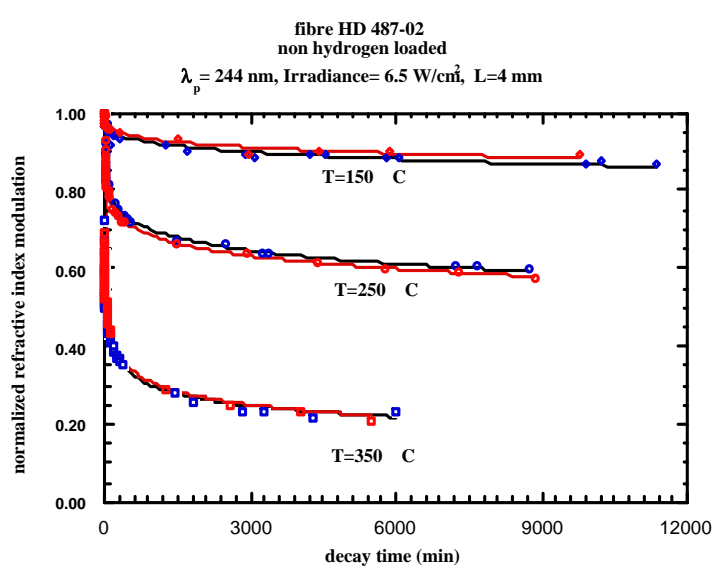

(e)

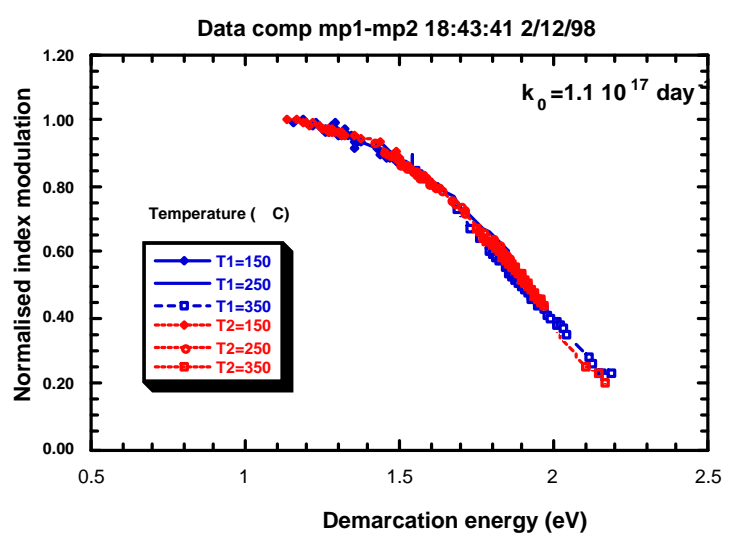

(f)

Figure 39. Set of isochrons and isotherm obtained by, reprinted with permission from [40]. Copyright 2000 University Lille 1. (a,b) compare isochrons obtained on the one hand and from one grating for each temperature, and on the other, with one grating by isochrones; (c) is the curve beam obtained after replotting, as mentioned in Section 3.1; (d) is the master curve deduced for the isochrones; (e,f) are isotherm experiments The same master curve is obtained after taking into account the temperature of the measurements.

Finally, it is possible to plot all results on the same plot using a demarcation and a suitable $k_{0}$ quantity, here we have used $k_{0}=1.1 \times 10^{17} \mathrm{day}^{-1}$. Using the same $k_{0}$ constant, it is also possible to rescale the isotherms and to superimpose isochrons and isotherms. This shows that the simple approach is applicable.

- Example of Equivalence Breaking

Example 1: non- $T$ dependent distribution against the $T$-dependent one.

Riant et al. [5]: non- $T$ dependent top hat function:

$$
\frac{\Delta n}{\Delta n_{0}}=1-\frac{E_{d}-E_{\min }}{E_{\max }-E_{\min }}, g(E)=\left\{\begin{array}{cl}
0, & E<E_{\min } \\
\frac{1}{E_{\max }-E_{\min }}, & E_{\min }<E<E_{\max } \\
0, & E_{\max }<E
\end{array}\right.
$$

Baker et al. [41]: T dependent top hat function:

$$
\frac{\Delta n}{\Delta n_{0}}=1-\frac{E_{d}-E_{\min }}{A k_{B} T}, g(E)=\left\{\begin{array}{cc}
0, & E<E_{\text {min }} \\
\frac{1}{A k_{B} T}, & E_{\min }<E<E_{\min }+A k_{B} T \\
0, & E_{\min }+A k_{B} T<E
\end{array}\right.
$$

These two interpretations can be differentiated by comparing isochrons and isotherms, there is $t, T$ equivalence in the first case, and non- $t,-T$ equivalence in the second case.

Example 2: occurrence of a secondary reaction: 
In the chosen experiment, described in Figure 40, refractive index change (RIC) is achieved by a $248 \mathrm{~nm}$ pulsed laser after $\mathrm{H}_{2}$ loading at a low $T$ high $P$. The isothermal measurements of a very long period (three years) have been plotted according to the demarcation energy with a rate constant of $1.4 \times 10^{12}$ day $^{-1}$. The distribution curve, which can be obtained by differentiation of the plotted curve for $E<1.7 \mathrm{eV}$, is, here, closer to a decreasing exponential. The drops at the end of the plot are relevant of another reaction with a much smaller rate constant $\left(90\right.$ day $\left.^{-1}\right)$ [40]. The figure, in the case of highly Ge-doped fiber core, shows that it is not possible to obtain a complete master curve due to another process occurring at the end of high-temperature treatment. There are two reactions, two $E_{d^{\prime}}$ are required, and a simple theory is not applicable (see Section 5.2).

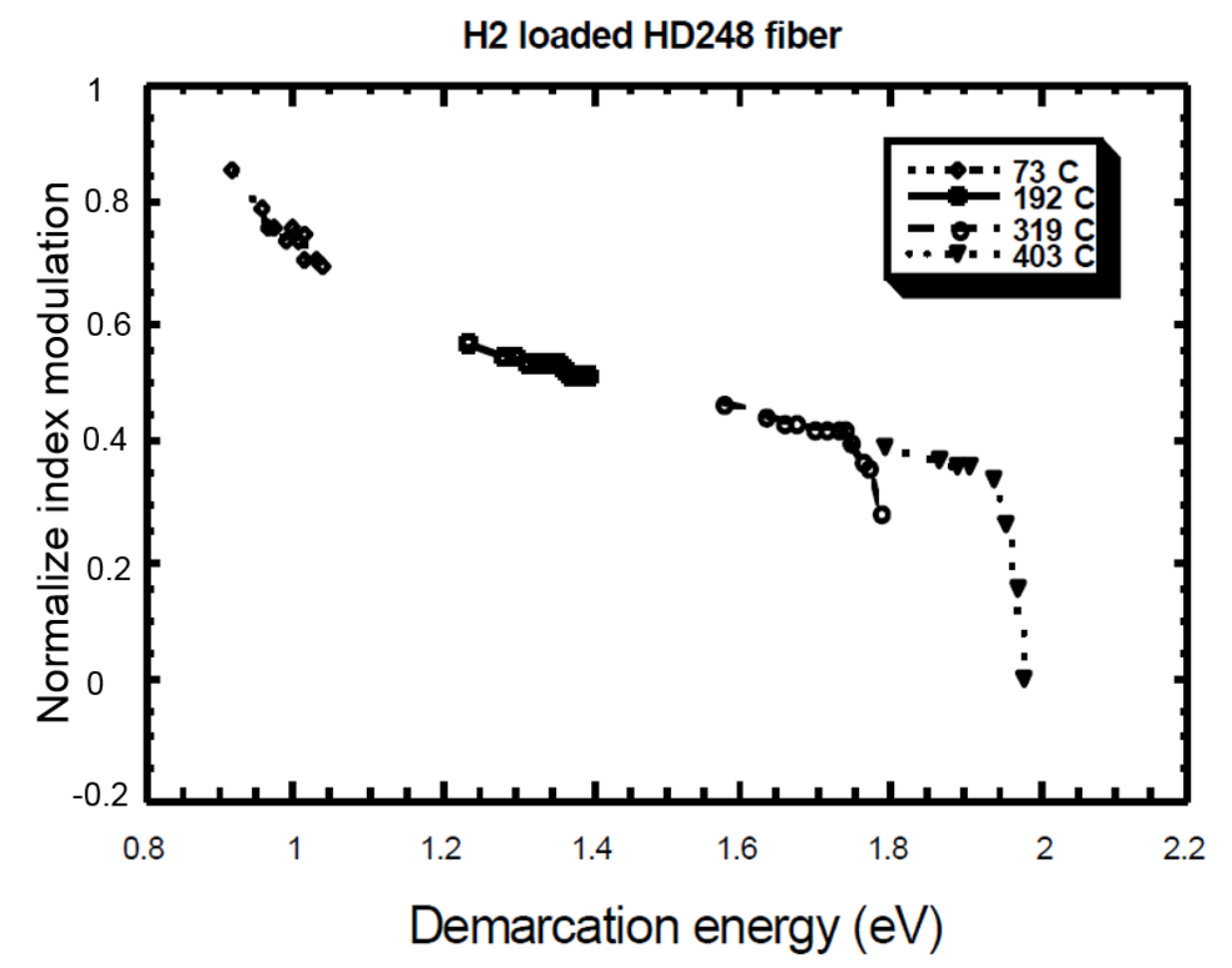

Figure 40. Result from [40]. Copyright 2000 University Lille 1. NICC vs. the demarcation energy.

\section{3. $k_{0}$ Comparison}

We have seen before that it is possible to get a master curve by providing only one parameter, i.e., $k_{0}$ when suitable assumptions are fulfilled. After obtaining $k_{0}$ and the master curve, we can compare the different stability functions (Figure 41). It is not necessary for this purpose to have a functional of $E_{d}$ for performing this comparison, but only the assumption of one active reaction. We can observe that the energies lie in the same range. This is due to a restriction effect of an observable property. The shapes of the curves are different, arising from different distribution functions. $k_{0}$ values, however, are quite different. The weight effect of vibrating species is not enough for explaining the observed range (as it is usual in solid). It is the probability for a cluster formation, i.e., a "transient complex". The larger the complex is, the smaller the probability is. Other examples can be found in Chabrerie [42], showing that $k_{0}$ can eventually depend on the effective trapping cross section with a $T^{2}$ dependence. In any case, $k_{0}$ gives information on the B species trapping mechanism. 


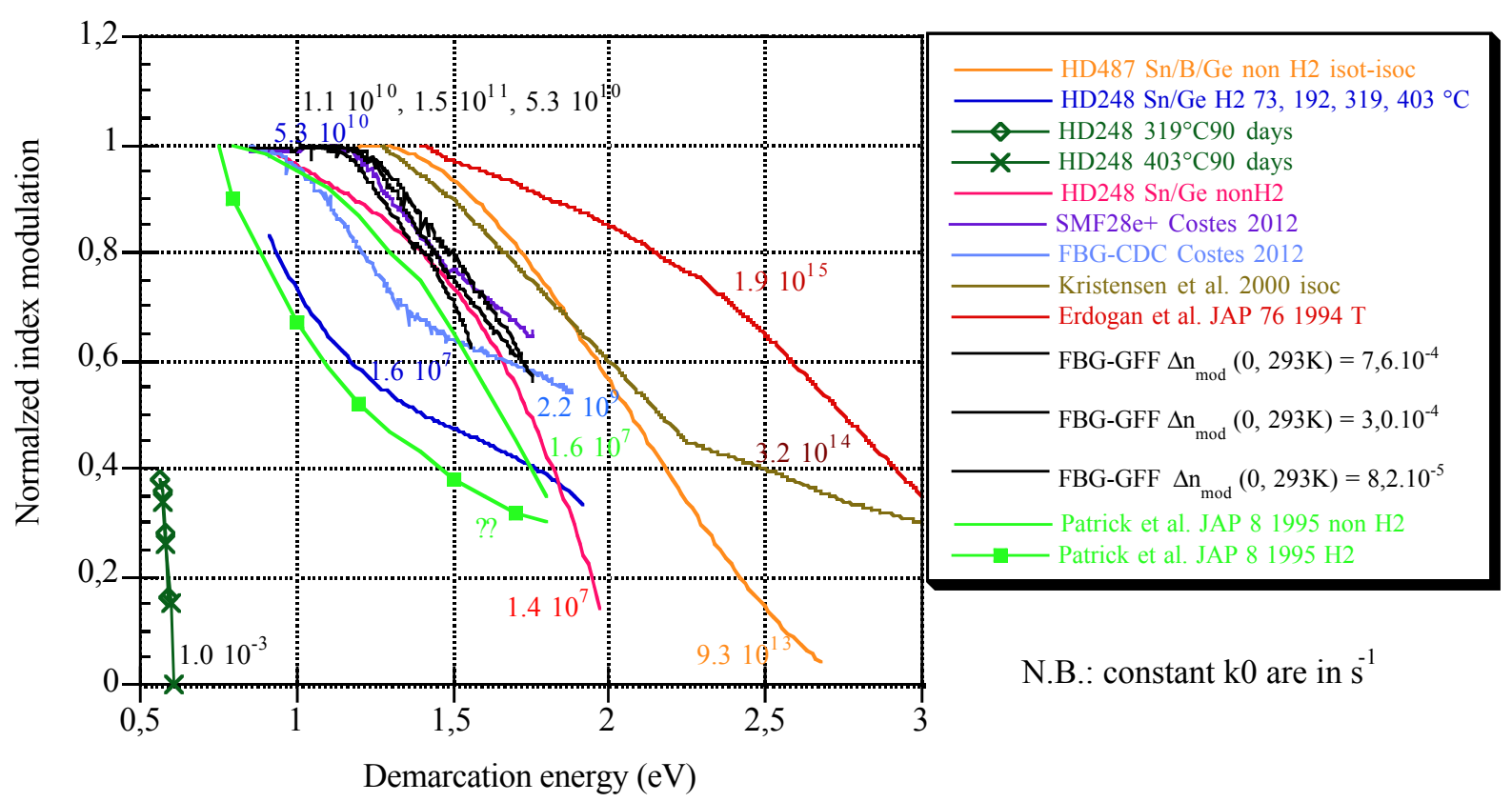

Figure 41. Shape of the stability function of a normalized index for different experimental conditions.

\section{Beyond Simple Theory}

\subsection{Higher Order Reaction}

This problem has been already mentioned by Primak [29]:

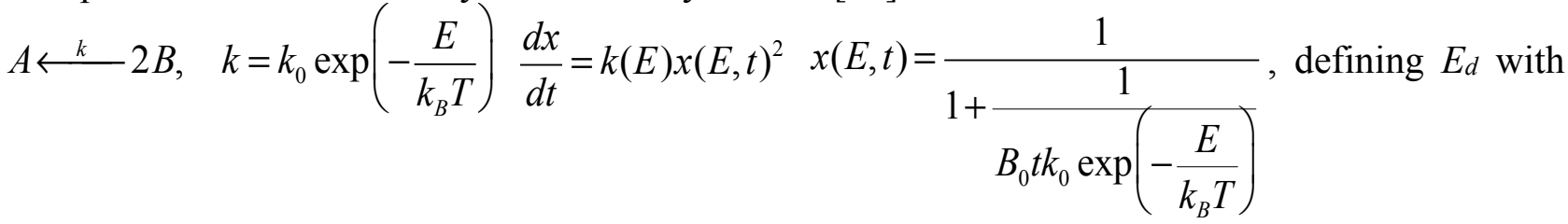
$x(E, t)=1 / 2$, we can compute $[B](t, T)$ if $B_{0}$ does not depend on $E$ in considering $x(E, t)=\frac{1}{1+\exp \left(\frac{E-E_{d}}{k_{B} T}\right)}$ as a rapidly varying function of $E$.

The $[B](t, T)=B_{0} \int_{0}^{\infty} x(E, t) g(E) d E$ and $[B]\left(E_{d}\right)=B_{0} \int_{E_{d}}^{\infty} g(E) d E$ as for first order, but with $E_{d}=k_{B} T \ln \left(B_{0} k_{0} T\right)$. The reaction properties are in $E_{d}$, and the disorder in $g(E)$, as before. However, $E_{d}$ depends on $B_{0}$, i.e., on the grating strength. The main conclusion is that stability, here, depends on the grating strength, even if forward and backward reactions are independent.

\subsection{Two Parallel Reactions}

Below are experimental results from Razafimhatratra et al. [40] in the case of $\mathrm{H}_{2}$-loaded fiber (Figure 42). A first $k_{0}$ can be found collapsing the curve at low $t$ and low $T$ (Figure 43). These master curves have been obtained with a rate constant equal to $1.4 \times 10^{12}$ day $^{-1}$. Another $k_{0}$ can be obtained when collapsing curves at large time or high temperature. 


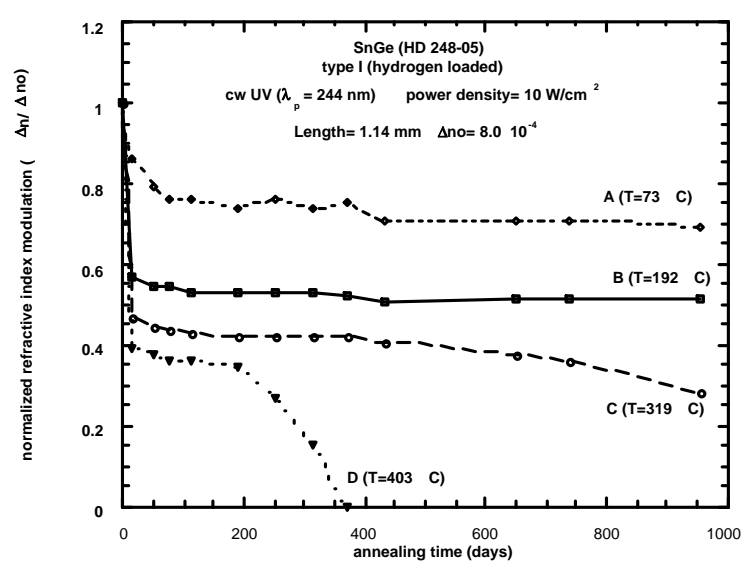

(a)

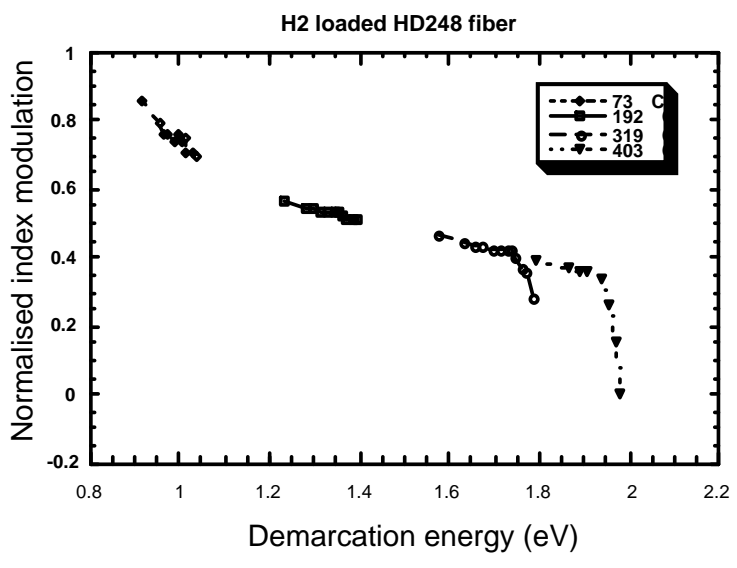

(b)

Figure 42. Results from [40], Copyright 2000 University Lille 1. The second master curve for large $t$ and $T$. Theory of serial reactions. (a) Isothermal annealing curves; (b) Master curve collapsing.

Index modulation at the end of the stability curve

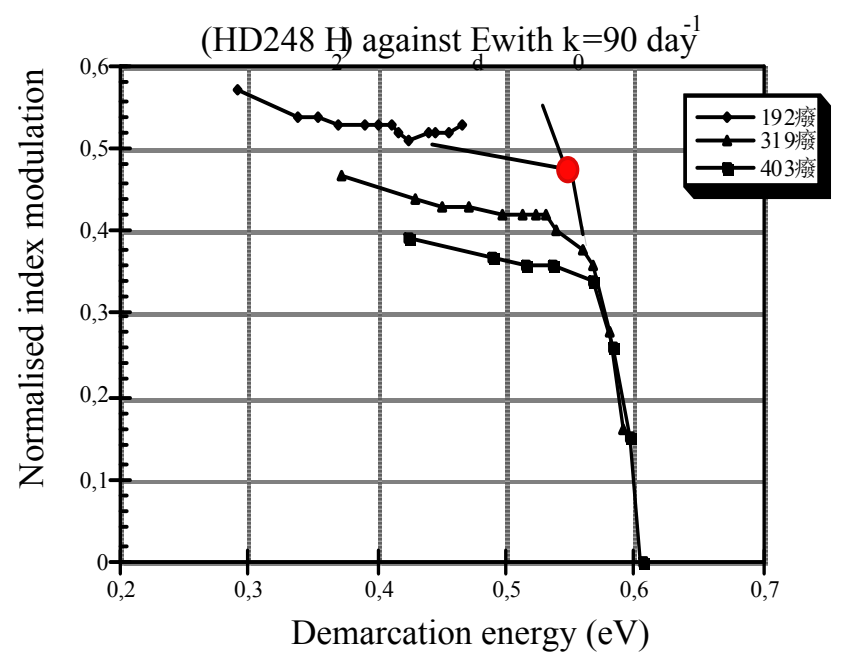

Figure 43. Corresponding master curve for low $t$ and low $T$.

The theory is as follows:

$$
\begin{aligned}
& A_{1} \stackrel{k_{1}}{\longleftarrow} B \frac{d \eta}{d t}=-\left(k_{1}\left(E_{1}\right)+k_{2}\left(E_{2}\right)\right) \eta \\
& \eta\left(E_{1}, E_{2}, t\right)=\exp \left[-\left(k_{1}\left(E_{1}\right)+k_{2}\left(E_{2}\right)\right) t\right] \\
& \frac{[B](t)}{B_{0}}=\int_{0}^{\infty} \int_{0}^{\infty} \exp \left[-\left(k_{1}\left(E_{1}\right)+k_{2}\left(E_{2}\right)\right) t\right] g\left(E_{1}\right) g\left(E_{2}\right) d E_{1} d E_{2} \\
& \frac{[B](t)}{B_{0}}=\int_{0}^{\infty} \exp \left[-k_{1}\left(E_{1}\right) t\right] g\left(E_{1}\right) d E_{1} \int_{0}^{\infty} \exp \left[-k_{2}\left(E_{2}\right) t\right] g\left(E_{2}\right) d E_{2}
\end{aligned}
$$




$$
\begin{gathered}
\frac{[B](t)}{B_{0}}=\int_{E_{d, 1}=k_{B} T \ln \left(k_{0,1} t\right)}^{\infty} g\left(E_{1}\right) d E_{1} . \quad \int_{E_{d, 2}=k_{B} T \ln \left(k_{0,2} t\right)}^{\infty} g\left(E_{2}\right) d E_{2} \\
E_{d, 1}=k_{B} T \ln \left(k_{1}^{0} t\right) ; \quad E_{d, 2}=k_{B} T \ln \left(k_{2}^{0} t\right)
\end{gathered}
$$

We note that isotherms and isochrons are, again, no longer equivalent. Let us illustrate the complex behavior of $\mathrm{B}$. We have chosen the constant rate $k_{0,2}$ as smaller than $k_{0,1}$. We note that $f\left(E_{d, 1}\right)$ and $f\left(E_{d, 2}\right)$ are different for a given $t, T$ couple. $E_{d, 1}$ and $E_{d, 2}$ do not have the same value (Figure $44 \mathrm{a}$ ).

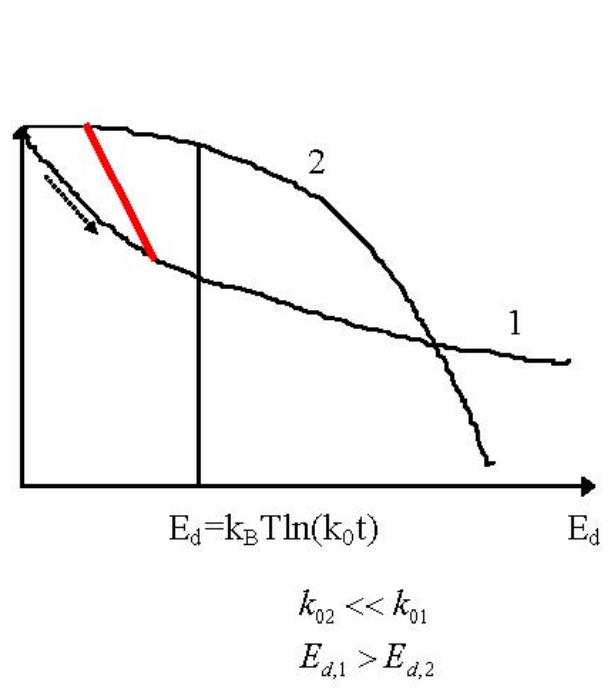

Isothermal description

$\mathrm{k}_{02}<<\mathrm{k}_{01}$

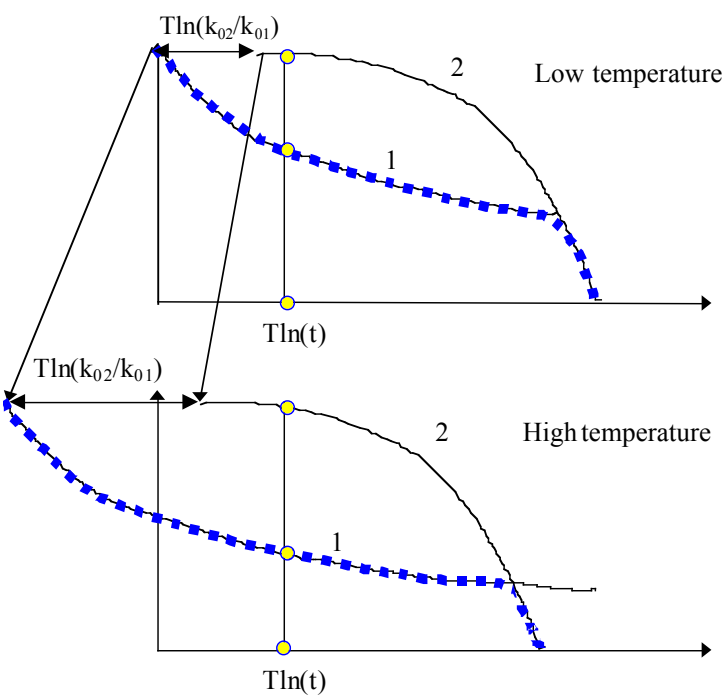

(a)

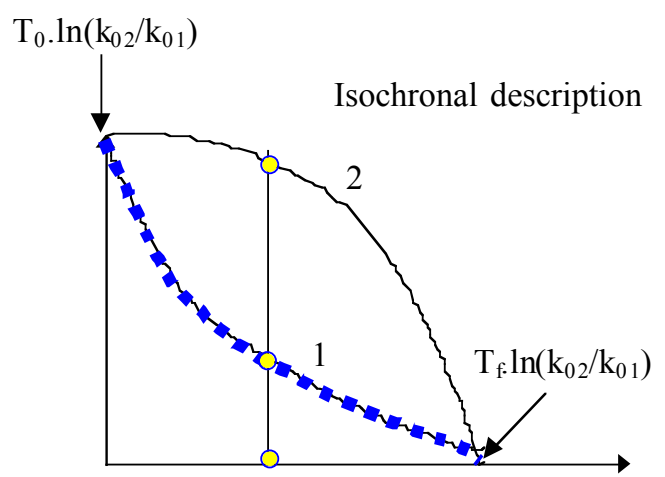

(b)

$\operatorname{Tln}(\Delta \mathrm{t})$

(c)

Figure 44. (a) Due to different $k$, the rate of evolution along reaction 1 or 2 are not the same; (b) Isothermal description. The dashed line shows the evolution of the erasure; (c) Isochronal description. Since the shift of the master curves increases as $T$ increases, the cross point can be not detectable.

In an $\ln t$ plot, the stability curves are shifted from each other (Figure $44 \mathrm{~b}$ ). The fastest reactions are predominant (curve 1) and we almost only see curve 1 behavior in the physical quantity. For a large $T$, the second behavior appears. At a larger $T$, curve 1 is shifted more on the left and curve 1 behavior is even 
more predominant in the behavior of the final physical quantity (Figure 44b). At a large $t$, curve 2 behavior appears but for a smaller contribution.

Along the isochronal curves in Figure $44 \mathrm{c}$, the temperature changes and the shift between curve 1 and 2 are increased in such a way that process 1 may have erased grating before process 2 appears. This may lead to an error on isothermal lifetime estimate.

\subsection{Serial Reactions}

Examples of serial reactions can be found in different fields, such as quasi elastic neutron scattering in PVC [43] or $E^{\prime}$ center production under X-ray in $\mathrm{SiO}_{2}$ [44], but we will stay in our field, considering the index change during writing after $\mathrm{H}_{2}$ loading of the glass. In this case, a sigmoidal curve is recorded according to the fluence (Figure 45, which is a linear-linear plot, shows a curve with an inflexion point, which is the indication of the occurrence of two serial reactions [45]). Moreover, in this example, the end of the curve reveals a non-exponential behavior.

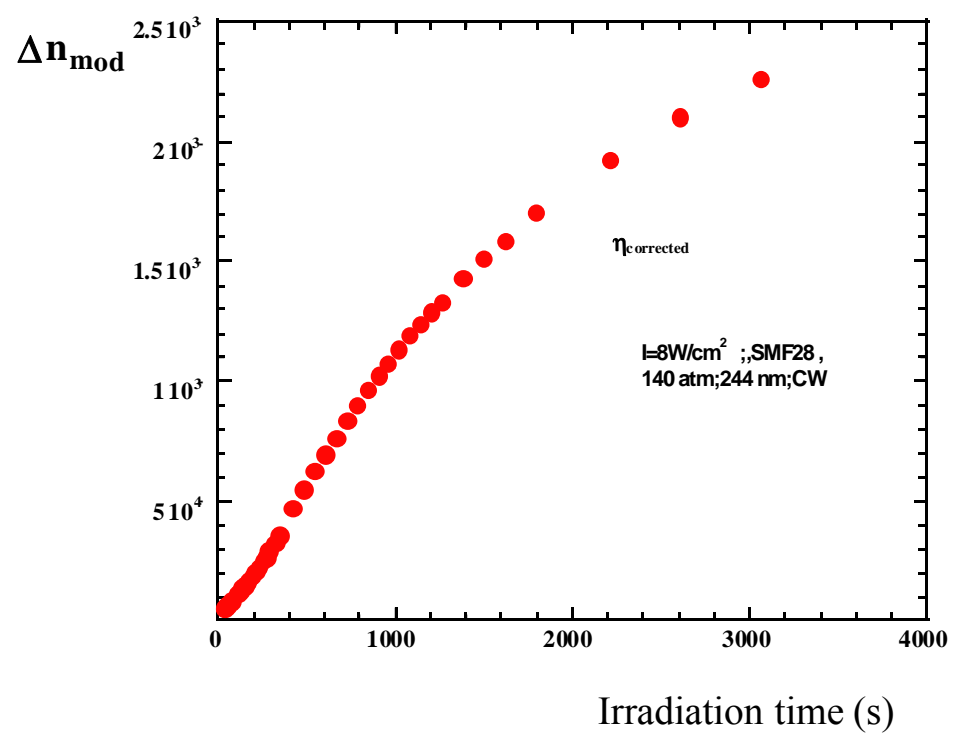

Figure 45. Index change in $\mathrm{H}_{2}$-loaded SMF28 (Corning) during writing with a CW $244 \mathrm{~nm}$ laser, reprinted with permission from [45]. Copyright 2001 University Lille 1.

- Theory of Serial Reactions

We consider the two successive reactions, $\mathrm{A} \stackrel{k_{1}}{\longrightarrow} \mathrm{B} \stackrel{k_{2}}{\longrightarrow} \mathrm{C}$. $\mathrm{C}$ is supposed to give rise to significant refractive index change. Note that, if one considers that $\mathrm{A}$ and $\mathrm{B}$ species are contributing to the refractive index change, it is then necessary to take them into account, but this does not cause any difficulties after solving the serial equation.

The solution of this equation, taking into account that the initial content is $A_{0}$, leads to the following expression for the advancement degree: $\eta=\mathrm{C} / A_{0}$

$$
\eta=\frac{1}{k_{2}-k_{1}}\left\{k_{2}\left[1-\exp \left(-k_{1} t\right)\right]-k_{1}\left[1-\exp \left(-k_{2} t\right]\right\}\right.
$$


We can consider that the first and the second activation energy barriers are thermally activated. Other cases will then be easily deduced. Thus, we have $k_{1}=k_{01} \exp \left(\frac{E_{1}}{k_{B} T}\right)$ and $k_{2}=k_{02} \exp \left(\frac{E_{2}}{k_{B} T}\right)$.

More symmetrically, the above equation can be rewritten as follows, isolating the energy term into the accolade:

$$
\eta=\frac{k_{1} k_{2}}{k_{2}-k_{1}}\left\{\frac{\left[1-\exp \left(-k_{1} t\right)\right]}{k_{1}}-\frac{\left[1-\exp \left(-k_{2} t\right]\right.}{k_{2}}\right\}
$$

We have to define one demarcation energy (because there is only one reaction, but in two steps) as usual, calculating the second derivative of $\eta$ according to $E_{1}$ for other fixed quantities $\left(t, T, E_{2}\right)$. As the $\eta$ function is symmetric according to the first reaction and the second reaction, the demarcation energy found by derivation, according to one, will also be the demarcation energy for the second one. Nevertheless, if we do this, the computation is heavy and does not lead to a simple expression. In fact, it is more efficient to study the $\eta$ function behavior and find an approximation. For this purpose, we will change the variable in order to suppress the time as we perform for more simple cases. This new variable will be an incomplete demarcation energy $E_{d}$, which the function study will allow completing. Thus, as there should be symmetry between the two steps of the reaction, we suppose $E_{d}=k_{B} T \ln \left(\frac{2 k_{01} k_{02}}{k_{01}+k_{02}} t\right)$, in other words, the smaller pre-exponential coefficient will dominate in the demarcation energy. Then, we can write $\exp \left[-t k_{0 i} \exp \left(-\frac{E_{i}}{k_{B} T}\right)\right]=\exp \left[-\exp \left(-\frac{E_{i}}{k_{B} T}+\ln \left(k_{0 i}\right)+\frac{E_{d}}{k_{B} T}-\ln \left(\frac{2 k_{01} k_{02}}{k_{01}+k_{02}}\right)\right)\right\rfloor$.

Finally, by setting $x=\frac{E_{d}}{k_{B} T}-\ln \left(\frac{2 k_{01} k_{02}}{k_{01}+k_{02}}\right), \quad x_{i}=-\frac{E_{i}}{k_{B} T}+\ln \left(k_{0 i}\right)$, we obtain the following expression for $\eta$ :

$$
\eta\left(x, x_{1}, x_{2}\right)=\frac{\exp \left(x_{1}+x_{2}\right)}{\exp \left(x_{2}\right)-\exp \left(x_{1}\right)}\left[\frac{1-\exp \left(\left(-\exp \left(x_{1}+x\right)\right)\right.}{\exp \left(x_{1}\right)}-\frac{1-\exp \left(\left(-\exp \left(x_{2}+x\right)\right)\right.}{\exp \left(x_{2}\right)}\right]
$$

When this function is plotted for a series of $x_{1}$ and $x_{2}$, separated by more than 2.3 (a factor 10 on the rate constants), the expression for $\eta\left(x, x_{1}, x_{2}\right)$ is the same as $1-\exp \left(\left(-\exp \left(x+\inf \left(x_{1}, x_{2}\right)\right)\right.\right.$.

Solving $[C](t, T)=A_{0} \int_{-\infty}^{+\infty} \int_{-\infty}^{+\infty} \eta\left(E_{1}, E_{2}, t, T\right) g_{1}\left(E_{1}\right) g_{2}\left(E_{2}\right) d E_{1} d E_{2}$, we will get one or the other of the two distributions, according to the relative magnitude of the $k_{0}$ 's and the relative position of the distributions along the abscise.

\subsection{Narrow and Steep Distribution Function}

The approximation of demarcation energy is based on the condition that the distribution function varies slower than $x(E, t, T)$ (see Section 2.2). On the contrary, it is no longer valid, especially as it is not directly applicable for a Dirac distribution function (SIREPA for single reaction pathway). However, it is possible to show that the approach can be extended. This simply needs to consider an 
experimental distribution function convoluted by the derivative of the advancement degree as described in Figure 46. Here is the demonstration:

(1) if we look for the experimental distribution by differentiation of B versus the demarcation energy in considering that it is possible to write $X(E, t, T)=X\left(E-E_{d}\right)$, we get $B=\int_{0}^{\infty} g(E) X\left(E-E_{d}\right) d E$. Then, we get the following: $\left.\frac{d B}{d E_{d}}=\int_{0}^{\infty} g(E) \frac{d\left[X\left(E-E_{d}\right)\right]}{d E_{d}} d E=-\int_{0}^{\infty} g(E) \frac{d X}{d E}\right)_{E-E_{d}} d E=\underbrace{\left[g *\left(-\frac{d X}{d E}\right)\right]_{E_{d}}}=\tilde{g}\left(E_{d}\right)$

The experimental distribution is the theoretical distribution broadened by $d X / d E$ (* means the convolution operator):

$$
\underbrace{\tilde{g}(E)}_{\begin{array}{c}
\text { experimental } \\
\text { distribution } \\
\text { function }
\end{array}}=\underbrace{\underbrace{g}_{\begin{array}{c}
\text { theoretical } \\
\text { distrubution }
\end{array}} *\left(-\frac{d X}{d E}\right)}_{\begin{array}{c}
\text { experimental } \\
\text { distribution is } \\
\text { the theoretical one } \\
\text { broadened by } \frac{\mathrm{dX}}{\mathrm{d}} \\
\text { width }=2 k_{B} T
\end{array}}
$$

where $X\left(E_{0}, E_{d}\right)=1-\exp \left(-\exp \left(\frac{E_{d}-E_{0}}{k_{B} T}\right)\right)$.

$$
\int_{0}^{\infty}-\frac{d X}{d E_{d}}\left(E-E_{d}\right) \delta\left(E-E_{0}\right) d E=-\frac{d X}{d E}\left(E_{0}-E_{d}\right)
$$

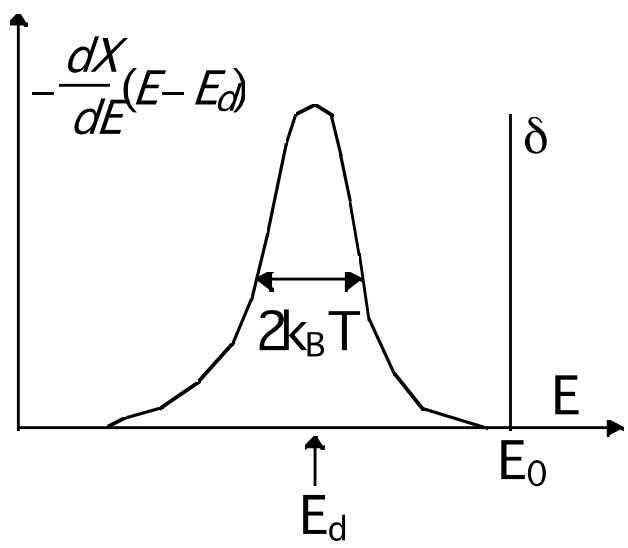

(a)

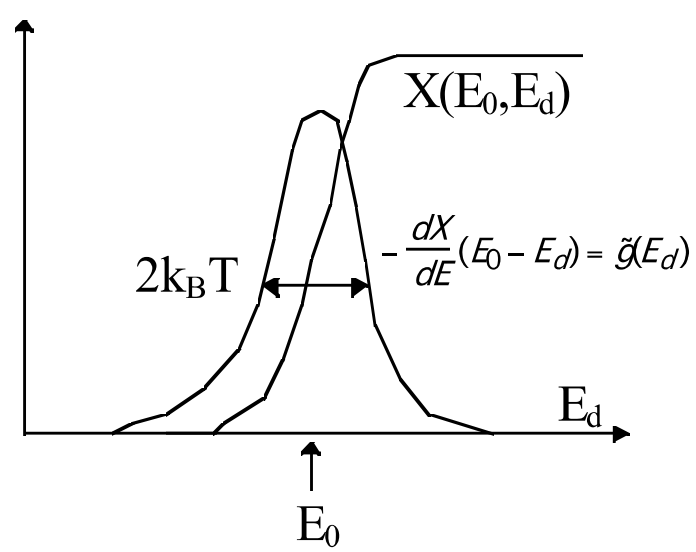

(b)

Figure 46. Derivative of the reaction advancement degree. (a) The two terms involved in the convolution; (b) the experimental distribution.

In particular, the Dirac function for a simple exponential will appear as a narrow bell shape curve with a width of $2 k_{B} T$.

(2) the inverse problem (to find the analytical function for B): 
If we consider $\left[g *\left(-\frac{d X}{d E}\right)\right]_{E_{d}}=\tilde{g}(E)$ and use the cutting method for computing the $\mathrm{B}$ integral, i.e., $[B]\left(E_{d}(t, T), T\right)=\int_{0}^{E_{d}(t, T)} \widetilde{g}(E, T) d E$. Are we going to find this following expression? $[B](t, T)=\int_{0}^{\infty} g(E) x(E, t, T) d E$.

In fact, we get the following:

$$
B\left(E_{d}\right)=\int_{0}^{E_{d}} \tilde{g}(E) d E=\int_{0}^{\infty} g(E) X\left(E-E_{d}\right) d E-\underbrace{\int_{0}^{\infty} g(E) X(E) d E}_{\begin{array}{c}
\approx 0 \text { if the distribution does } \\
\text { not touch zero energy. }
\end{array}}
$$

In conclusion, the VAREPA approach can be applied to any distribution performing at the end of a deconvolution. For instance, in the case of a square distribution (Figure 47), such as when the low Ge-doped fiber is hydrogen loaded, the edge of the distribution will be smoothed, giving rise at the beginning of the reaction to a simple exponential behavior on time.
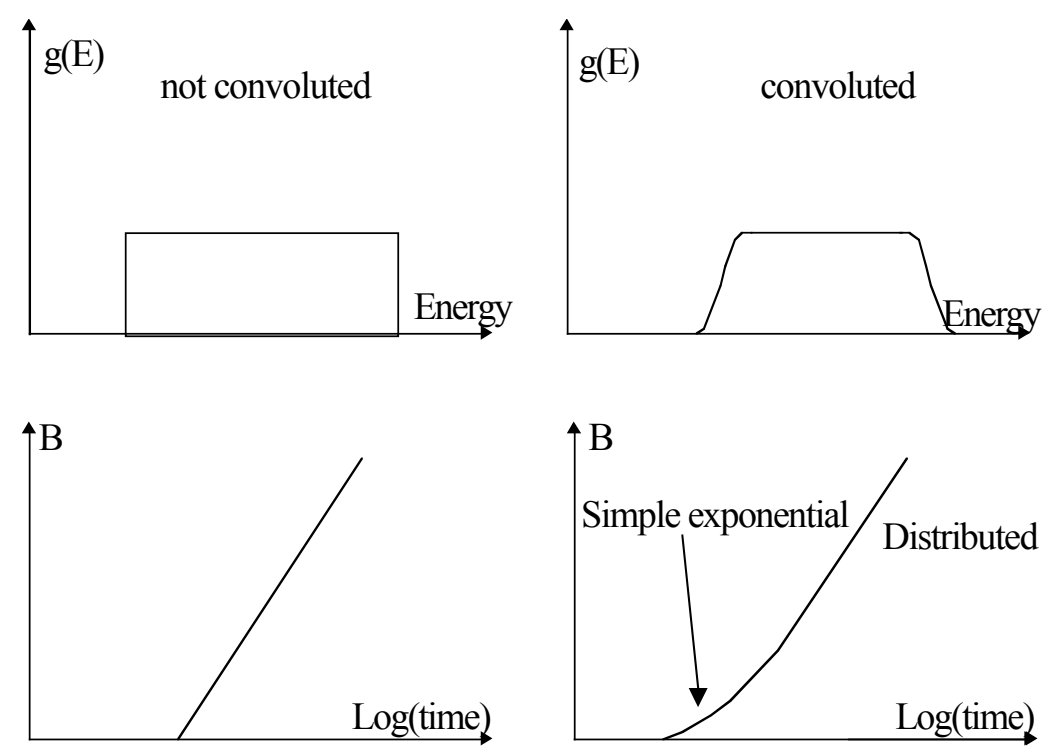

Figure 47. Effective distribution function.

\subsection{Distributed Non-Activated Kinetics (Photochromism, Luminescence)}

\subsection{1. "Writing like"}

The equation of excitation is the following one: $\frac{d A^{*}}{d t}=\frac{\varepsilon(\mathrm{h} v) A P}{\mathrm{~h} v}$, where $P$ is the intensity. We can note that, if $\varepsilon(h v)$ (the absorption cross section) is varying rapidly on $h v$ (edge absorption), we can use the VAREPA approach to compute the excited state concentration, excited under white light. 


$$
A \underset{\mathrm{hv}_{f}}{\stackrel{\mathrm{h} v_{a}}{\longrightarrow}} A^{*}, \frac{d A^{*}}{d t}=\frac{\varepsilon\left(\mathrm{h} v_{a}\right) A P}{\mathrm{~h} v}, \text { with } \varepsilon\left(\mathrm{h} v_{a}\right)=\varepsilon_{0} \exp \left(\frac{\mathrm{h} v-\mathrm{h} v_{a}}{\Delta}\right)
$$

More generally, we have $\frac{d x}{d t}=k(1-x), \quad x=1-\exp (-k t), k=k\left(E / E_{0}(\zeta)\right)$, where $E$ is the distributed variable with $g(E)$ as the distributed function, $\xi$ is the variable related to the energy source, and $E_{0}$ is a function of this variable with the same dimension as the distributed variable.

We get:

$$
\left.\left[A^{*}\right](t, \zeta)=A_{0} \int_{0}^{E_{d}} g(E) d E, E_{d} \text { defined as } \frac{\partial^{2} x}{\partial E^{2}}\right)_{t, \zeta}\left(E=E_{d}\right)=0 \Rightarrow E_{d}(t, \zeta)
$$

It is worth noting that the distribution is characterized by the shape of the distribution and a width that we can find, again fixing a value of $\xi$ as $\xi_{0}$.

\subsubsection{Erasure}

The erasure corresponds to luminescence. The intensity of this is proportional to $-\frac{d A^{*}}{d t}$. If the stability of the luminescence site is distributed, the lifetime will increase on time. In that case, we have:

$$
\frac{d A^{*}}{d t}=-k \cdot A^{*}, k=k_{0} \exp \left(-\frac{E}{k_{B} T}\right)
$$

this is the same as in Section 2.3 and the analysis procedure is the same.

\section{Real Analysis of Bragg Grating Writing and Stability}

In analysis of the refractive index changes, photo-induced in fiber Bragg gratings, we have to pay attention that we work on index modulation and average index changes, but not on point one. However, all theories developed above are for point index change. The difference between these two quantities is a Fourier transform along $z$, i.e., along Power density fluctuations. To make the link between them, it is, therefore, necessary to know the relationship between $k_{0} t$ and $P$. Is $k_{0}$ proportional to $P$ or to $P^{2}$ ?

The determination of this $k_{0}$ dependence has been recently reviewed in Reference [46]. Of course, non-linear dependence on $P$ combined with the non-linear behavior on $k_{0} t$ will lead to a distortion of the interference pattern and to bandwidth broadening. However, this is not the only detrimental effect. Recalling the conclusion of Section 2.3.4, the stability of inter-fringe will be smaller than the one at the maximum of the bright UV fringes. Therefore, during a burn-in process, non- $\mathrm{H}_{2}$ loading fibers made by a pulsed laser (for instance) can exhibit a broadening.

\section{Practical Formalism to Describe Generalized Thermally Activated Processes}

It is worth noting that a general approach, applicable to any activation energy process analyzed by any experimental measurement (isothermal, isochronous or tempering), has been published by Miller et al. [32]. These authors demonstrate a numerical solving of the generalized equation in which 
$k_{0}, T$, or the energy reference can be time dependent. Again, they use a cutting energy, which is defined by $\left.\frac{\partial^{2} x}{\partial E \partial t}\right|_{E=E_{d}}=0$.

Specifically, for tempering, the demarcation energy is defined by an expression quite different than for isothermal experiments.

$$
\frac{E_{d}}{k_{B} T}+\ln \left(\frac{E_{d}}{k_{B} T}+2\right)=\ln \left(k_{0} c k_{B} T\right)
$$

where $c$ is defined by $T(t)=\frac{t}{c k_{B}}+T_{0}[42]$.

\section{Conclusions}

We have shown a development of a framework for modeling the thermally activated processes, either for creating or erasing species, such as the ones involved in UV-induced refractive index changes [46], based on distributed activation energy of a physico-chemical reaction. From experimental measurements (isochrons and isotherms), this framework allows to find the distribution function, providing only a constant, which can be determined by a simple variable change when a few assumptions are verified. From this distribution, it is possible to know the complete time behavior and to determine the annealing conditions for extending lifetimes.

This approach can be used for other physical quantities, e.g., photodarkening, stress relaxation, and luminescence decay. Finally, let us mention that the framework developed here for the time evolution of an activated energy process can be used for analyzing the frequency behavior of similar system. For instance, Perlmutter et al. [47] describe quasi-elastic light-scattering spectrum analysis in the range of 0.1 to $100 \mathrm{MHz}$, in term of energy barrier distribution of sites similar to a Poisson distribution. The demarcation energy analog here is $k T \ln \left(k_{0} / \omega\right)$.

\section{Acknowledgments}

We are very grateful to Pierre Niay and Marc Douay for many discussions and the availability of their measurements. I thank also Christian Chabrerie and Sylvain Costes for their important thesis.

\section{Author Contributions}

Bertrand Poumellec interprets the experimental data and wrote the paper. Matthieu Lancry and Bertrand Poumellec conceived, devised and helped carry out the experiments, edited the paper and suggested some new directions.

\section{Conflicts of Interest}

The authors declare no conflict of interest. 


\section{References}

1. Erdogan, T.; Mizrahi, V.; Lemaire, P.J.; Monroe, D. Decay of ultraviolet-induced fiber Bragg gratings. J. Appl. Phys. 1994, 76, 73-80.

2. Ishikawa, S.; Inoue, A.I.; Harumoto, M. Adequate aging condition for fiber Bragg grating based on simple power law model, Optical Fiber Communication Conference and Exhibit, 1998. OFC '98, San Jose, CA, USA, 22-27 February 1998.

3. Richert, R.; Blumen, A. Disorder Effects on Relaxational Processes; Springer Verlag: New York, NY, USA, 1994.

4. Bernage, P.; Taunay, T.; Leconte, B.; Douay, M.; Niay, P.; Bayon, J.F.; Poignant, H.; Herlemont, H.; Legrand, J.; Poumellec, B.; et al. Inscription Kinetics and Thermal Stability of Bragg Gratings Written within Heated Fibers. In Proceedings of the Doped Fiber Devices and systems II, Denver, CO, USA, 20 November 1996.

5. Riant, I.; Poumellec, B. Thermal decay of gratings written in hydrogen-loaded germanosilicate fibers. Electron. Lett. 1998, 34, 1603-1604.

6. Lancry, M.; Poumellec, B.; Costes, S.; Magné, J.; Reliable Lifetime Prediction for Passivated Fiber Bragg Gratings for Telecommunication Applications. Fibers 2014, 2, 92-107.

7. Xie, W.X.; Niay, P.; Bernage, P.; Douay, M.; Taunay, T.; Bayon, J.F.; Delevaque, E.; Monnerie, M. Photoinscription of Bragg gratings within preform plates of high NA germanosilicate fibers: Searching for an experimental evidence of type IIA photosensitivity in preform plates. Opt. Commun. 1996, 124, 295-300.

8. Poumellec, B.; Niay, P.; Douay, M.; Bayon, J.F. The UV induced refractive index grating in $\mathrm{Ge}_{-} \mathrm{SiO}_{2}$ preforms: Additional $\mathrm{CW}$ experiments and the macroscopic origin of the change in index. J. Phys. D Appl. Phys. 1996, 29, 1842-1856.

9. Fiori, C.; Devine, R.A.B. Ultraviolet irradiation induced compaction and photoetching in amorphous, thermal $\mathrm{SiO}_{2}$. MRS Proc. 1986, 61, 187-195.

10. Primak, W.; Kampwirth, R. The radiation compaction of vitreous silica. J. Appl. Phys. 1968, 39, 5651-5658.

11. Allan, C.D.; Smith, C.; Borelli, N.F.; Seward, T.P., III. 193-nm excimer laser induced densification of fused silica. Opt. Lett. 1996, 21, 1960-1962.

12. Lemaire, P.J. Reliability of optical fibers exposed to hydrogen: Prediction of long-term loss increases. Opt. Eng. 1991, 30, 780-789.

13. Marcerou, J.F.; Février, H.; Gabla, P.M.; Augé, J. Sensitivity of erbium-doped fibers to hydrogen: Implications for long-term system performance. In Proceedings of the Optical Fiber Communication'94, San Jose, CA, USA, 20-25 February 1994.

14. Beales, K.J.; Cooper, K.J.; Rush, J.D. Increased attenuation in optical fibers caused by diffusion of molecular hydrogen at room temperature. Electron. Lett. 1983, 19, 917-919.

15. Smith, C.L. A theory of transient creep in metals. Proc. Phys. Soc. 1948, 61, 201-205.

16. Poirier, M.; Thibault, S.; Lauzon, J.; Ouellette, F. Dynamics and orientational behaviour of U.V. induced luminescence bleaching in Ge-doped silica optical fiber. Opt. Lett. 1993, 18, 870-872.

17. Erdogan, T.; Mizrahi, V.; Lemaire, P.J.; Monroe, D. Decay of U.V.-induced fiber Bragg gratings. In Proceedings of the Optical Fiber Communication'94, San Jose, CA, USA, 20-25 February 1994. 
18. Kyung, J.H.; Lawandy, N.M. Direct observation of the effective $\chi^{(2)}$ grating in bulk glasses encoded for second-harmonic generation. Opt. Lett. 1996, 21, 632-634.

19. Friebele, E.J.; Gingerich, M.E.; Griscom, D.L. Survivability of optical fibers in space. In Proceedings of the Optical Materials Reliability and Testing, Boston, MA, USA, country, 8 September 1992.

20. Deparis, O.; Griscom, D.L.; Mégret, P.; Decréton, M.; Blondel, M. Influence of the cladding thickness on the evolution of the $\mathrm{NbOHC}$ band in optical fibers exposed to gamma radiations. J. Non Cryst. Solids 1996, 216, 124-128.

21. Rodriguez, V.D.; Rodriguez-Mendoza, U.R.; Martin, I.R.; Lavin, V.; Nunez, P. Site distribution in $\mathrm{Cr}^{3+}$ and $\mathrm{Cr}^{3+}-\mathrm{Tm}^{3+}$ doped alkaline silicate glasses. In Proceedings of the $\mathrm{SiO}_{2}$ and Advanced Dielectrics, L’Aquila, Italy, 15-17 June 1997.

22. Monroe, D.; Kastner, M.A. Exactly exponential band tail in a glasssy semiconductor. Phys. Rev. $B$ 1986, 33, 8881-8884.

23. Kroide, M. Apparent viscosity and relaxation mechanism of glasses below glass transition temperature. Phys. Chem. Glasses 1997, 38, 83-86.

24. Phillips, J.C. Kohlrausch relaxation and glass transitions in experiment and in molecular dynamics simulations. J. Non Cryst. Solids 1995, 182, 155-161.

25. Mohanna, Y.; Saugrain, J.-M.; Rousseau, J.-C.; Ledoux, P. Relaxation of internal stresses in optical fibers. J. Lightwave Technol. 1990, 8, 1799-1802.

26. Lemaire, P.J.; Tomita, A. Behavior of single mode MCVD fibers exposed to hydrogen. In Proceedings of the European Conference on Optical Communications, Stuttgart, Germany, 3-6 September 1984.

27. Kohlrausch, R. Ueber das Dellmann'sche Elektrometer. Ann. Phys. 1847, 72, 353-405. (In German)

28. Vand, V. A theory of the irreversible electrical resistance changes of metallic films evaporated in vacuum. Proc. Phys. Soc. Lond. 1943, A55, 222-246.

29. Primak, W. Kinetics of Processes distributed in activation energy. Phys. Rev. B 1955, 100, 1677-1689.

30. Primak, W. Large temperature range annealing. J. Appl. Phys. 1960, 31, 1524-1533.

31. Orenstein, J.; Kastner, M. Photocurrent transient spectroscopy: Measurement of the density of localized states in a-AS $\mathrm{SSe}_{3}$. Phys. Rev. Lett. 1981, 46, 1421-1424.

32. Miller, S.L.; McWhorter, P.J.; Miller, W.M.; Dressendorfer, P.V. A practical predictive formalism to describe generalised activated physical processes. J. Appl. Phys. 1991, 70, 4555-4568.

33. Lemaire, P.J.; Monroe, D.P.; Watson, H.A. Hydrogen-induced-loss increases in erbium-doped amplifier fibers: Revised predictions. In Proceedings of the Optical Fiber Communication'94, San Jose, CA, USA, 20-25 February 1994.

34. Vandenbrink, J.P. Master stress relaxation function of silica glasses. J. Non Cryst. Solids 1996, 196, 210-215.

35. Kannan, S.; Lemaire, P.J.; Guo, J.; LuValle, M.J. Reliability predictions on fiber gratings through alternate methods. In Proceedings of the Bragg Gratings Photosensitivity, and Poling in Glass Fibers and Waveguides: Applications and Fundamentals, Williamsburg, VA, USA, 26-28 October 1997.

36. Poumellec, B. Links between writing and erasure (or stability) of Bragg gratings in disordered media. J. Non Cryst. Solids 1998, 239, 108-115. 
37. Razafimahatratra, D.; Thesis, P. Etude de la Stabilité de la Variation D'indice de Réfraction Photoinduite par Insolation Laser Dans les Guides D'onde Optiques Germanosilicates. PhD Thesis, University of Sciences and Technologies of Lille, Lille, France, 2000. (In French)

38. Costes, S. Extension de L'approche par la Courbe Maîtresse de la Prédiction des Durées de vie de Réseaux D'indice Complexes Inscrits par UV dans les Fibres; NNT: 2013PA112102. Ph.D. Thesis, Université Paris Sud, Orsay, France, 2013. (In French)

39. Patrick, H.; Gilbert, S.L. Growth of Bragg gratings produced by continuous-wave ultraviolet light in optical fiber. Opt. Lett. 1993, 18, 1484-1486.

40. Razafimahatratra, D.; Niay, P.; Douay, M.; Poumellec, B.; Riant, I. Comparison of isochronal and isothermal decays of Bragg gratings written through continuous-wave exposure of an unloaded germanosilicate fiber. Appl. Opt. 2000, 39, 1924-1933.

41. Baker, S.R.; Rourke, H.N.; Baker, V.; Goodchild, D. Thermal decay of fiber Bragg gratings written in Boron and Germanium codoped silica fiber. J. Lightwave Technol. 1997, 15, 1470-1477.

42. Chabrerie, C. De L'utilisation des Recuits Isothermes et Isochrones pour la Caractérisation de Structures MOS Irradiées. Ph.D. Thesis, Paris 7 University, Paris, France, 1997. (In French)

43. Ngai, K.L.; Roland, C.M.; Greaves, G.N. An interpretation of quasielastic neutron scattering and molecular dynamics simulation results on the glass transition. J. Non Cryst. Solids 1995, 182, 172-179.

44. Mashkov, V.A.; Austin, W.R.; Zhang, L.; Leisure, R.G. Fundamental role of creation and activation in radiation-induced defect production in high-purity amorphous $\mathrm{SiO}_{2}$. Phys. Rev. Lett. 1996, 76, 2926-2929.

45. Ramecourt, D.; Niay, P.; Bernage, P.; Riant, I.; Douay, M. Growth of strength in $\mathrm{H}_{2}$ loaded telecommunication fiber during CW UV post-exposure. Electron. Lett. 1999, 35, 329-330.

46. Lancry, M.; Poumellec, B. UV laser processing and multiphoton absorption processes in optical telecommunication fiber materials. Phys. Rep. 2013, 523, 207-229.

47. Perlmutter, S.H.; Levenson, M.D.; Shelby, R.M.; Weissman, M.B. Polarization properties of quasielastic light scattering in fused-silica optical fiber. Phys. Rev. B 1990, 42, 5294-5305.

(C) 2015 by the authors; licensee MDPI, Basel, Switzerland. This article is an open access article distributed under the terms and conditions of the Creative Commons Attribution license (http://creativecommons.org/licenses/by/4.0/). 